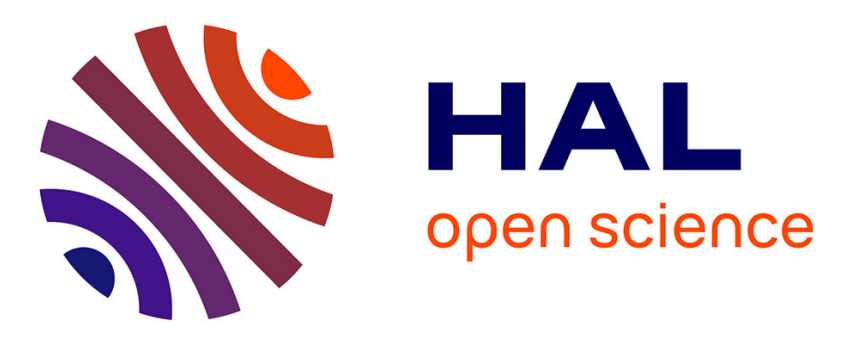

\title{
A low Mach correction able to deal with low Mach acoustics
}

\author{
Pascal Bruel, Simon Delmas, Jonathan Jung, Vincent Perrier
}

\section{To cite this version:}

Pascal Bruel, Simon Delmas, Jonathan Jung, Vincent Perrier. A low Mach correction able to deal with low Mach acoustics. Journal of Computational Physics, 2019, 378, pp.723-759. 10.1016/j.jcp.2018.11.020 . hal-01953424

\section{HAL Id: hal-01953424 \\ https://hal.inria.fr/hal-01953424}

Submitted on 12 Dec 2018

HAL is a multi-disciplinary open access archive for the deposit and dissemination of scientific research documents, whether they are published or not. The documents may come from teaching and research institutions in France or abroad, or from public or private research centers.
L'archive ouverte pluridisciplinaire HAL, est destinée au dépôt et à la diffusion de documents scientifiques de niveau recherche, publiés ou non, émanant des établissements d'enseignement et de recherche français ou étrangers, des laboratoires publics ou privés. 


\title{
A low Mach correction able to deal with low Mach acoustics
}

\author{
Pascal Bruel*, Simon Delmas ${ }^{\dagger}$, Jonathan Jung ${ }^{\ddagger}$ and Vincent Perrier ${ }^{\S}$
}

\section{Contents}

1 The accuracy problem of the Roe scheme at low Mach number and some low Mach corrections

1.1 Behavior of the continuous equations in the low Mach number limit . . . . . . . 4

1.2 The finite volume Roe scheme . . . . . . . . . . . . . . . . . . . . . 4

1.3 Analysis of the Roe scheme when the Mach number goes to $0 \ldots \ldots$. . . . . . 5

1.3.1 Expression of the Roe scheme in our case . . . . . . . . . . . . . 5

1.3.2 Behaviour in the low Mach number limit . . . . . . . . . . . . 5

1.4 Some low Mach corrections . . . . . . . . . . . . . . . . . . . . . 6

1.4.1 The fix of Dellacherie et al. [12] . . . . . . . . . . . . . . 6

1.4.2 The fix of Rieper [47] . . . . . . . . . . . . . . . . . . . . . . . 7

1.4 .3 The Roe-Turkel scheme $[23] \ldots \ldots \ldots \ldots$

2 Impact of the low Mach number corrections on the computation of an acoustic wave in a low Mach number flow

2.1 Two-scale dimensionless equations: acoustic limit on an incompressible flow . . . . 9

2.2 Acoustic wave propagation in a low Mach number flow . . . . . . . . . . . . . . . 10

2.2 .1 Test case description . . . . . . . . . . . . . . . . . . 10

2.2.2 Numerical results with the Roe scheme and with some low Mach number fixes 10

2.3 Analysis . . . . . . . . . . . . . . . . . . . . . . . 12

2.3.1 Two scales dimensionless study of the Roe-Turkel scheme . . . . . . . . . . 14

2.3.2 Two-scale dimensionless study of the Roe scheme and the Roe scheme corrected with the Dellacherie et al. or Rieper fixes . . . . . . . . . . . 15

3 Construction of a new scheme: accurate in the low Mach number limit for $\begin{array}{lr}\text { steady and for acoustics computations } & \mathbf{1 7}\end{array}$

3.1 Development of a scheme for the wave system $(38) \ldots \ldots \ldots \ldots$

3.1.1 General expression of the new scheme . . . . . . . . . . . . . 18

3.1.2 Conditions for ensuring a semi-discrete energy inequality . . . . . . . . 19

3.1.3 Accuracy at low Mach number in the steady case . . . . . . . . . . . . 20

3.1.4 Stability analysis of the explicit new scheme in dimension one . . . . . . 20

3.1.5 Stability analysis of the explicit new scheme in dimension two . . . . . . . 23

3.1.5.1 Modified equation on a Cartesian mesh in dimension two . . . . . 23

3.1.5.2 Stability analysis of the explicit new scheme in dimension two for a one dimensional flow on a regular Cartesian mesh . . . . . . . . 23

3.1.6 Final expression of the new scheme for the wave equation . . . . . . . 25

3.2 From the linear wave equation to the barotropic Euler equations . . . . . . . . . 25

3.2.1 Dimensionless wave equation . . . . . . . . . . . . . . 25

3.2.2 The dimensionless new Roe scheme in the subsonic case . . . . . . . . . . 26

${ }^{*}$ CNRS / Univ Pau \& Pays Adour/ E2S UPPA, Laboratoire de Mathématiques et de leurs Applications de Pau - Fédération IPRA, UMR5142 64000, Pau, France and Cagire team, Inria Bordeaux Sud-Ouest, France pascal.bruel@univ-pau.fr.

${ }^{\dagger}$ CNRS / Univ Pau \& Pays Adour/ E2S UPPA, Laboratoire de Mathématiques et de leurs Applications de Pau - Fédération IPRA, UMR5142 64000, Pau, France and Cagire team, Inria Bordeaux Sud-Ouest, France simon.delmas@inria.fr.

${ }^{\ddagger}$ CNRS / Univ Pau \& Pays Adour/ E2S UPPA, Laboratoire de Mathématiques et de leurs Applications de Pau - Fédération IPRA, UMR5142 64000, Pau, France and Cagire team, Inria Bordeaux Sud-Ouest, France jonathan.jung@univ-pau.fr.

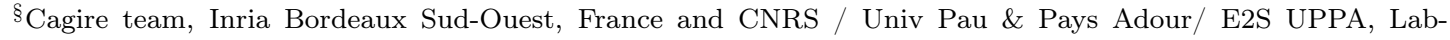
oratoire de Mathématiques et de leurs Applications de Pau - Fédération IPRA, UMR5142 64000, Pau, France, vincent.perrier@inria.fr. 
3.2.3 The new fix for the Roe scheme in the subsonic case expressed in original

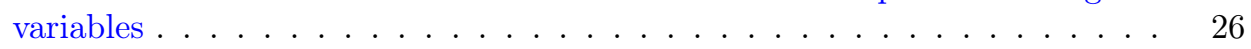

3.2.4 Practical implementation of the new fix . . . . . . . . . . . . . . 27

3.2.5 Implementation of wall boundary conditions . . . . . . . . . . . . . . . . . 27

4 Numerical results $\quad 28$

4.1 Numerical results on the linear wave equation . . . . . . . . . . . . . . . . . 28

4.1 Test case . . . . . . . . . . . . . . . . . . . 28

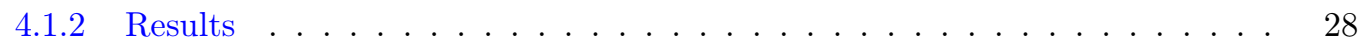

4.2 Numerical results on the barotropic Euler equation . . . . . . . . . . . . . . . . 28

4.2.1 Computation of an acoustic wave in a one dimensional low Mach number flow 30

4.2.2 Computation of an acoustic wave in a two dimensional low Mach number flow 30

4.2.3 Computation of a steady low Mach number flow . . . . . . . . . . . . . 31

4.2.3.1 Test case description . . . . . . . . . . . . . . . . . . . . . . . . . . . . . . . 33

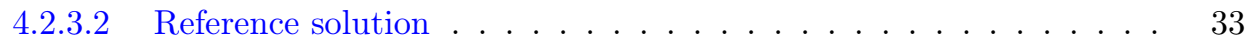

4.2.3.3 Numerical results . . . . . . . . . . . . . . . . . . . . . . . . . . . . . . . . . 33

4.2 .4 Transonic airfoil . . . . . . . . . . . . . . . . . . 33

\begin{abstract}
This article deals with acoustic computations in low Mach number flows with density based solvers. For ensuring a good resolution of the low Mach number base flow, a scheme able to deal with stationary low Mach number flows is necessary. Previously proposed low Mach number fixes are tested with acoustic computations. Numerical results prove that they are not accurate for acoustic computations. The issues raised with acoustic computations with low Mach number fixes are discussed, and a new scheme is developed, in order to be accurate not only for steady low Mach number flows, but also for acoustic computations. Numerical tests show the improvement of the proposed scheme with respect to the state of the art.
\end{abstract}

\title{
Introduction
}

In this article, we are interested in the barotropic Euler system

$$
\left\{\begin{array}{l}
\partial_{t} \rho+\nabla \cdot(\rho \mathbf{u})=0, \\
\partial_{t}(\rho \mathbf{u})+\nabla \cdot(\rho \mathbf{u} \otimes \mathbf{u})+\nabla p=0
\end{array}\right.
$$

where the pressure law $p=p(\rho)$ satisfies the monotonicity and the convex property. For the sake of simplicity, we will suppose that $p$ follows a $\gamma$ law, i.e.

$$
p(\rho)=\kappa \rho^{\gamma}
$$

with $\kappa>0$ and $\gamma>1$. Under these assumptions, this system is well-known to be hyperbolic with eigenvalues given by $\lambda_{ \pm}=\mathbf{u} \cdot \mathbf{n} \pm a$, where the sound speed $a$ is given by $a=\sqrt{\mathbf{d}_{\rho} p(\rho)}$, with genuinely nonlinear characteristic fields and $\lambda=\mathbf{u} \cdot \mathbf{n}$ of multiplicity $d-1$ where $d \in\{1,2,3\}$ is the space dimension, with linear characteristic fields. Equations (1) can be written in the conservative form

$$
\partial_{t} \mathcal{U}+\nabla \cdot \mathbf{f}(\mathcal{U})=0
$$

where $\mathcal{U}=(\rho, \rho \mathbf{u})^{T}$ is the vector of conservative variables and $\mathbf{f}$ is the flux, given in dimension $d$ by

$$
\mathbf{f}(\mathcal{U})=\left(\begin{array}{c}
\rho \mathbf{u}^{T} \\
\rho \mathbf{u} \otimes \mathbf{u}+p \mathrm{I}
\end{array}\right) .
$$

Given one time scale $t_{0}$, one length scale $x_{0}$ and one density scale $\rho_{0}$, the following dimensionless variables may be defined

$$
\tilde{t}=\frac{t}{t_{0}}, \tilde{\mathbf{x}}=\frac{\mathbf{x}}{x_{0}}, \tilde{\rho}=\frac{\rho}{\rho_{0}} .
$$

It is natural to scale the velocity by $u_{0}=x_{0} / t_{0}$, and the pressure by $p_{0}=\kappa \rho_{0}^{\gamma}$. If the corresponding dimensionless variables are used instead of the original ones, the following system is obtained

$$
\left\{\begin{array}{l}
\partial_{\tilde{t}} \tilde{\rho}+\nabla_{\tilde{\mathbf{x}}} \cdot(\tilde{\rho} \tilde{\mathbf{u}})=0 \\
\partial_{\tilde{t}}(\tilde{\rho} \tilde{\mathbf{u}})+\nabla_{\tilde{\mathbf{x}}} \cdot(\tilde{\rho} \tilde{\mathbf{u}} \otimes \tilde{\mathbf{u}})+\frac{1}{\gamma M^{2}} \nabla_{\tilde{\mathbf{x}}} \tilde{p}=0
\end{array}\right.
$$


with $\tilde{\mathbf{u}}=\mathbf{u} / u_{0}, \tilde{p}=p / p_{0}$ and $\frac{1}{\gamma M^{2}}=\frac{t_{0} p_{0}}{\rho_{0} u_{0} x_{0}}$. It can be reformulated as

$$
\frac{1}{\gamma M^{2}}=\frac{t_{0} p_{0}}{\rho_{0} u_{0} x_{0}}=\frac{p_{0}}{\rho_{0} u_{0}^{2}}=\frac{\kappa \rho_{0}^{\gamma}}{\rho_{0} u_{0}^{2}}=\frac{\kappa \rho_{0}^{\gamma-1}}{u_{0}^{2}}=\frac{a_{0}^{2}}{\gamma u_{0}^{2}} .
$$

We are interested in the regime when $M \rightarrow 0$. This kind of regime may be encountered in low velocity compressible flows $\left(u_{0} \ll a_{0}\right)$, or in nearly incompressible flows $\left(a_{0} \rightarrow \infty\right)$ [45]. Low Mach number flows are also a natural concern when dealing with multiphase compressible flows, in which the sound velocity of the liquid phase is high [30, 43, 44].

From a theoretical point of view, this singular limit was studied in [28]. From a numerical point of view, the approximation of (5) raises several problems. In the literature, the following problems have been extensively discussed

- Accuracy problems It is known (and we will recall that in section 1) that in the low Mach number limit, the pressure perturbations scales as $\mathcal{O}\left(M^{2}\right)$, whereas most of the schemes give a spurious mode in $\mathcal{O}(M)[23,46]$. Several fixes have been proposed, see [23, 31, 33, 11, 47, $10,12,41,8,25]$. Note that if the mesh is unstructured, and composed of simplicial cells (triangles in $2 \mathrm{~d}$, tetrahedra in $3 \mathrm{~d}$ ), the accuracy with the Roe scheme is recovered [48, 21, 13]. Higher order aspects, based on the discontinuous Galerkin method, have been dealt with in $[4,38,39]$. See also [42] for an example in the shallow water context, and [22] for a review on the accuracy problem of compressible solvers in the low Mach number limit.

- Time integration strategy In a compressible flow, the acoustic waves in a direction $\mathbf{n}$ are traveling at a velocity $\mathbf{u} \cdot \mathbf{n} \pm a$, where $a$ is the sound velocity, whereas entropic waves are traveling at $\mathbf{u} \cdot \mathbf{n}$. When the Mach number is low, the ratio between the acoustic and convective wave velocities is high, which gives a CFL number for explicit schemes much lower than the CFL number based on the convection velocity. In this case, implicit-explicit strategies have been proposed [29, 40, 44, 15, 24].

This article is focused on fixing the accuracy problem. The accuracy problem was extensively discussed in the one-scale steady problem. However, fixing low Mach number problems may raise other problems, for example

- A violation of entropy inequality (see [6] for an entropic version of [23]),

- An inaccuracy in the gravity waves, see [3] for an example with Euler model with gravity.

- A degraded CFL stability condition with explicit time stepping [5].

In this article, we are focused not only on accurately computing a low Mach number flow with a compressible method, but also on accurately computing the acoustic waves in it. A real life application is the stability study of jets in cross flow used for effusion cooling in combustion chambers under acoustic perturbations induced by combustion instabilities [17]. In this context, it is necessary to develop a method that

1. accurately solves the time averaged low Mach number flow,

2. accurately solves the acoustic perturbations of the time averaged flow,

3. is high order, because this is necessary for unsteady turbulent simulations (Large Eddy Simulation, or Direct Numerical Simulation).

To the best of our knowledge, if point 1 has already been extensively addressed, point 2 has only been dealt with pressure based algorithms [36] but never with a density based solver as proposed in this article. Moreover, the discussion on the high order aspects was addressed for steady simulations [4], but has never been addressed for the computation of acoustic perturbations.

This article is organized as follows. In section 1, the origins of the accuracy problem in the steady case are recalled, the analysis first proposed in [23] is explained, and some previously proposed fixes are presented. In section 2, the problem of acoustic propagation in a low Mach number flow is tackled. The equations of acoustic perturbations are derived and a test case of the propagation of an acoustic wave in a low Mach number flow is developed. Then, state of the art low Mach number fixes are tested and proved to be inaccurate in that case. An analysis of the origin of such an inaccuracy is proposed. Based on the fixes proposed in section 1, and on the problems raised in section 2, a new scheme is designed in section 3 for being accurate for both steady computations and acoustic computations. In section 4 , the new scheme is tested and compared with other schemes on steady and unsteady configurations. 


\section{The accuracy problem of the Roe scheme at low Mach num- ber and some low Mach corrections}

\subsection{Behavior of the continuous equations in the low Mach number limit}

In this section, the results proven theoretically in [28] are formally recalled. We are interested in the solutions of (5) when $M \rightarrow 0$. All the variables of the system, $\varphi \in\{\rho, \mathbf{u}\}$ are developed as expansions in power of the Mach number $M$ :

$$
\tilde{\varphi}(\tilde{\mathbf{x}}, \tilde{t} ; M)=\sum_{n=0}^{N} M^{n} \tilde{\varphi}^{(n)}(\tilde{\mathbf{x}}, \tilde{t})+\mathcal{O}\left(M^{N+1}\right) .
$$

By injecting these quantities in (5), the momentum equation at order $M^{-2}$ and $M^{-1}$ gives

$$
\nabla_{\tilde{\mathbf{x}}} \tilde{p}^{(0)}=\nabla_{\tilde{\mathbf{x}}} \tilde{p}^{(1)}=0
$$

and then, since $p$ is a regular function of $\rho$,

$$
\nabla_{\tilde{\mathbf{x}}} \tilde{\rho}^{(0)}=\nabla_{\tilde{\mathbf{x}}} \tilde{\rho}^{(1)}=0 .
$$

This leads to

$$
\tilde{\rho}^{(0)}(\tilde{\mathbf{x}}, \tilde{t})=\tilde{\rho}^{(0)}(\tilde{t}) \quad \text { and } \quad \tilde{\rho}^{(1)}(\tilde{\mathbf{x}}, \tilde{t})=\tilde{\rho}^{(1)}(\tilde{t}) .
$$

At order $M^{0}$, we get

$$
\left\{\begin{array}{l}
\partial_{\tilde{t}} \tilde{\rho}^{(0)}+\nabla_{\tilde{\mathbf{x}}} \cdot\left(\tilde{\rho}^{(0)} \tilde{\mathbf{u}}^{(0)}\right)=0, \\
\partial_{\tilde{t}}\left(\tilde{\rho}^{(0)} \tilde{\mathbf{u}}^{(0)}\right)+\nabla_{\tilde{\mathbf{x}}} \cdot\left(\tilde{\rho}^{(0)} \tilde{\mathbf{u}}^{(0)} \otimes \tilde{\mathbf{u}}^{(0)}\right)+\frac{1}{\gamma} \nabla_{\tilde{\mathbf{x}}} \tilde{p}^{(2)}=0 .
\end{array}\right.
$$

If the initial and boundary conditions are well prepared [28], which means that the initial and boundary conditions on $\rho^{(1)}$ are equal to 0 , then $\rho^{(1)}$ is uniformly and constantly equal to 0 . This can be expressed as

$$
\tilde{\rho}(\tilde{\mathbf{x}}, \tilde{t}, M)=\tilde{\rho}^{(0)}(\tilde{t})+M^{2} \tilde{\rho}^{(2)}(\tilde{\mathbf{x}}, \tilde{t})+\mathcal{O}\left(M^{3}\right) .
$$

\subsection{The finite volume Roe scheme}

Defining the Roe average as

$$
\rho_{i j}=\sqrt{\rho_{i} \rho_{j}}, \quad \mathbf{u}_{i j}=\frac{\sqrt{\rho_{i}} \mathbf{u}_{i}+\sqrt{\rho_{j}} \mathbf{u}_{j}}{\sqrt{\rho_{i}}+\sqrt{\rho_{j}}} \quad \text { and } \quad a_{i j}^{2}= \begin{cases}\frac{\Delta p}{\Delta \rho}, & \text { if } \Delta \rho \neq 0, \\ a\left(\rho_{i}\right), & \text { otherwise }\end{cases}
$$

where $\Delta(\cdot)=(\cdot)_{j}-(\cdot)_{i}$. Then the Roe scheme for the barotropic equations is defined as

$$
\left|\Omega_{i}\right| \mathbf{d}_{t} \mathcal{U}_{i}(t)+\sum_{\Gamma_{i j} \subset \partial \Omega_{i}}\left|\Gamma_{i j}\right| \Phi_{i j}^{\mathrm{Roe}}=0
$$

with

$$
\begin{aligned}
\Phi_{i j}^{\text {Roe }}=\frac{\mathbf{f}\left(\mathcal{U}_{i}\right)+\mathbf{f}\left(\mathcal{U}_{j}\right)}{2} \cdot \mathbf{n}_{i j} & -\frac{1}{4}\left|\mathbf{u}_{i j} \cdot \mathbf{n}_{i j}-a_{i j}\right|\left(\Delta \rho-\frac{\rho_{i j}}{a_{i j}} \Delta\left(\mathbf{u} \cdot \mathbf{n}_{i j}\right)\right)\left(\begin{array}{c}
1 \\
\mathbf{u}_{i j}-a_{i j} \mathbf{n}_{i j}
\end{array}\right) \\
& -\frac{1}{2}\left|\mathbf{u}_{i j} \cdot \mathbf{n}_{i j}\right| \rho_{i j}\left(\begin{array}{c}
0 \\
\Delta \mathbf{u}^{\perp}\left(\mathbf{n}_{i j}\right)
\end{array}\right) \\
& -\frac{1}{4}\left|\mathbf{u}_{i j} \cdot \mathbf{n}_{i j}+a_{i j}\right|\left(\Delta \rho+\frac{\rho_{i j}}{a_{i j}} \Delta\left(\mathbf{u} \cdot \mathbf{n}_{i j}\right)\right)\left(\begin{array}{c}
1 \\
\mathbf{u}_{i j}+a_{i j} \mathbf{n}_{i j}
\end{array}\right)
\end{aligned}
$$

where

$$
\mathbf{u}^{\perp}(\mathbf{n}):=\mathbf{u}-(\mathbf{u} \cdot \mathbf{n}) \mathbf{n}
$$

denotes the tangential component of $\mathbf{u}$ with respect to normal direction $\mathbf{n}$. 


\subsection{Analysis of the Roe scheme when the Mach number goes to 0}

The Roe scheme is known to give wrong results for stationary low Mach number flows with quadrangular meshes [23]: tests show that pressure fluctuations are much larger than the expected ones. For explaining these results, we briefly recall the analysis of [23], based on a discrete asymptotic expansion.

\subsubsection{Expression of the Roe scheme in our case}

In the case we are interested in (subsonic case), using (12) gives

$$
\left\{\begin{array}{c}
\mathbf{d}_{t} \rho_{i}+\frac{1}{\left|\Omega_{i}\right|} \sum_{\Gamma_{i j} \subset \partial \Omega_{i}}\left|\Gamma_{i j}\right|\left[\frac{\rho_{i} \mathbf{u}_{i}+\rho_{j} \mathbf{u}_{j}}{2} \cdot \mathbf{n}_{i j}+\frac{\rho_{i j}}{2 a_{i j}}\left(\mathbf{u}_{i j} \cdot \mathbf{n}_{i j}\right)\left(\mathbf{u}_{i}-\mathbf{u}_{j}\right) \cdot \mathbf{n}_{i j}\right. \\
\left.+\frac{a_{i j}}{2}\left(\rho_{i}-\rho_{j}\right)\right]=0, \\
\mathbf{d}_{t}\left(\rho_{i} \mathbf{u}_{i}\right)+\frac{1}{\left|\Omega_{i}\right|} \sum_{\Gamma_{i j} \subset \partial \Omega_{i}}\left|\Gamma_{i j}\right|\left[\frac{\rho_{i}\left(\mathbf{u}_{i} \cdot \mathbf{n}_{i j}\right) \mathbf{u}_{i}+\rho_{j}\left(\mathbf{u}_{j} \cdot \mathbf{n}_{i j}\right) \mathbf{u}_{j}}{2}\right. \\
+\frac{a_{i j}}{2}\left(\rho_{i}-\rho_{j}\right)\left[\mathbf{u}_{i j}+\left(\mathbf{u}_{i j} \cdot \mathbf{n}_{i j}\right) \mathbf{n}_{i j}\right] \\
+\frac{\rho_{i j}}{2}\left|\mathbf{u}_{i j} \cdot \mathbf{n}_{i j}\right|\left(\mathbf{u}_{i}^{\perp}\left(\mathbf{n}_{i j}\right)-\mathbf{u}_{j}^{\perp}\left(\mathbf{n}_{i j}\right)\right) \\
+\frac{\rho_{i j}\left(\mathbf{u}_{i j} \cdot \mathbf{n}_{i j}\right)}{2 a_{i j}}\left[\left(\mathbf{u}_{i}-\mathbf{u}_{j}\right) \cdot \mathbf{n}_{i j}\right] \mathbf{u}_{i j} \\
\left.+\left[\frac{p_{i}+p_{j}}{2}+\frac{\theta_{i j} \rho_{i j} a_{i j}}{2}\left(\mathbf{u}_{i}-\mathbf{u}_{j}\right) \cdot \mathbf{n}_{i j}\right] \mathbf{n}_{i j}\right]=0
\end{array}\right.
$$

where $\theta_{i j}=1, p_{k}=p\left(\rho_{k}\right)$ and the states $(\cdot)_{i j}$ are defined by $(10)$. (13) will be used for expressing the fixes.

Expressed with dimensionless variables (4), and considering

$$
\tilde{a}_{i j}=\frac{a_{i j}}{a_{0}}, \quad\left|\tilde{\Gamma}_{i j}\right|=\frac{\left|\Gamma_{i j}\right|}{\left|\Gamma_{0}\right|}, \quad\left|\tilde{\Omega}_{i}\right|=\frac{\left|\Omega_{i}\right|}{\left|\Omega_{0}\right|}
$$

where $\left|\Gamma_{0}\right| /\left|\Omega_{0}\right|=1 / L$. The dimensionless version of(12) is given by

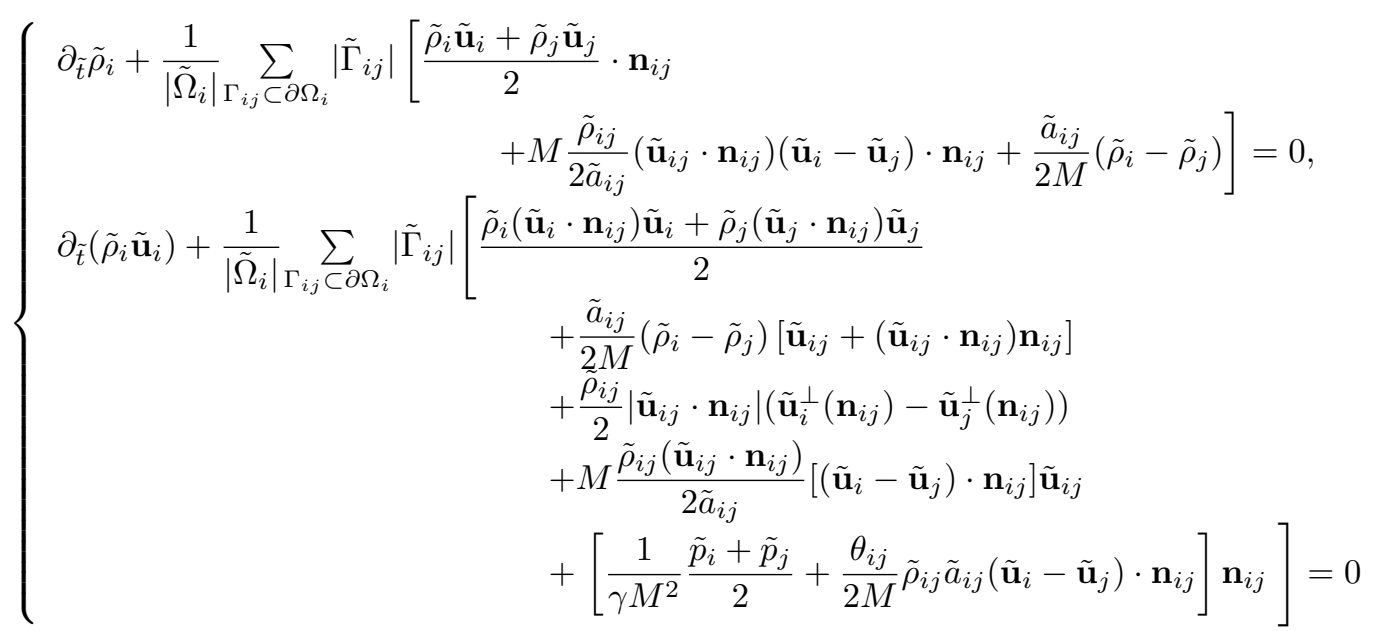

where $\theta_{i j}=1$ for the Roe scheme. For more details, we refer to [12]. (15) will be used for performing the asymptotic analysis of the scheme.

\subsubsection{Behaviour in the low Mach number limit}

It is well known that in the low Mach number limit, $\tilde{\rho}^{(0)}$ is uniform [23, 12, 22]. Moreover, in the same references, it is proved that $\left(\tilde{p}^{(1)}, \tilde{\mathbf{u}}^{(0)}\right)_{i}$ satisfies

$$
\left\{\begin{array}{c}
\sum_{\Gamma_{i j} \subset \partial \Omega_{i}}\left|\tilde{\Gamma}_{i j}\right|\left[-\tilde{\rho}^{(0)} \tilde{a}^{(0)}\left(\tilde{\mathbf{u}}_{i}^{(0)}-\tilde{\mathbf{u}}_{j}^{(0)}\right) \cdot \mathbf{n}_{i j}+\left(\tilde{p}_{i}^{(1)}-\tilde{p}_{j}^{(1)}\right) / \gamma\right]=0 \\
\sum_{\Gamma_{i j} \subset \partial \Omega_{i}}\left|\tilde{\Gamma}_{i j}\right|\left[-\delta \tilde{\rho}^{(0)} \tilde{a}^{(0)}\left(\tilde{\mathbf{u}}_{i}^{(0)}-\tilde{\mathbf{u}}_{j}^{(0)}\right) \cdot \mathbf{n}_{i j}+\left(\tilde{p}_{i}^{(1)}-\tilde{p}_{j}^{(1)}\right) / \gamma\right] \mathbf{n}_{i j}=0
\end{array}\right.
$$


with $\delta=1$. Then, two situations are distinguished:

- In the triangular mesh case, the system (16) is a square system that has a nonzero determinant $[48,21,22]$. Its single solution is 0 , which gives for all cells

$$
-\tilde{\rho}^{(0)} \tilde{a}^{(0)}\left(\tilde{\mathbf{u}}_{i}^{(0)}-\tilde{\mathbf{u}}_{j}^{(0)}\right) \cdot \mathbf{n}_{i j}+\left(\tilde{p}_{i}^{(1)}-\tilde{p}_{j}^{(1)}\right) / \gamma=0 .
$$

Then, as proved in [21], we get that $\left(\tilde{p}^{(1)}, \tilde{\mathbf{u}}^{(0)}\right)_{i}$ satisfies

$$
\forall i, \quad \forall j \in \nu(i), \quad\left\{\begin{array}{l}
\tilde{p}_{i}^{(1)}=\tilde{p}_{j}^{(1)} \\
\tilde{\mathbf{u}}_{i}^{(0)} \cdot \mathbf{n}_{i j}=\tilde{\mathbf{u}}_{j}^{(0)} \cdot \mathbf{n}_{i j}
\end{array}\right.
$$

where $\nu(i)$ is the set of the index $j$ such that $\Omega_{i}$ and $\Omega_{j}$ are neighbor cells. Then, if on the inlet and outlet boundaries we have $\tilde{p}^{(1)}=0$, we get that $\tilde{p}^{(1)}=0$ over the whole domain. Then, $\tilde{p}-\tilde{p}^{(0)}=\mathcal{O}\left(M^{2}\right)$ and there is no accuracy problem on triangular meshes.

- In the general case, as explained in [23, 22], the system (16) admits non-zero solutions implying that the discrete solution can contain a non constant pressure fluctuation $p^{(1)}$, which is the spurious mode that can be numerically observed as a wrong order of magnitude of the pressure fluctuations in a steady low Mach case. This problem on Cartesian or quadrangular meshes is often referred to as the "accuracy problem at low Mach number" of the Roe scheme.

\subsection{Some low Mach corrections}

In this part, three classical low Mach number corrections, representative of those available in the litterature, are described and we explain from an analytical point of view why they cure the accuracy problem at low Mach number on Cartesian or quadrangular meshes.

\subsubsection{The fix of Dellacherie et al. [12]}

The all-Mach correction of Dellacherie et al. [12] consists in replacing the term $\Delta(\mathbf{u} \cdot \mathbf{n})$ in the momentum equation (13b) of the Roe scheme by the term $\min \left(M_{i j}, 1\right) \Delta(\mathbf{u} \cdot \mathbf{n})$, where $M_{i j}$ is a term of the order of the Mach number computed at each edge of the mesh. In the future numerical tests, we will take $M_{i j}=\max \left(M_{i}, M_{j}\right)=\max \left(\left\|\mathbf{u}_{i}\right\| / a_{i},\left\|\mathbf{u}_{j}\right\| / a_{j}\right)$. More precisely, in the subsonic case (13), the scheme reads as

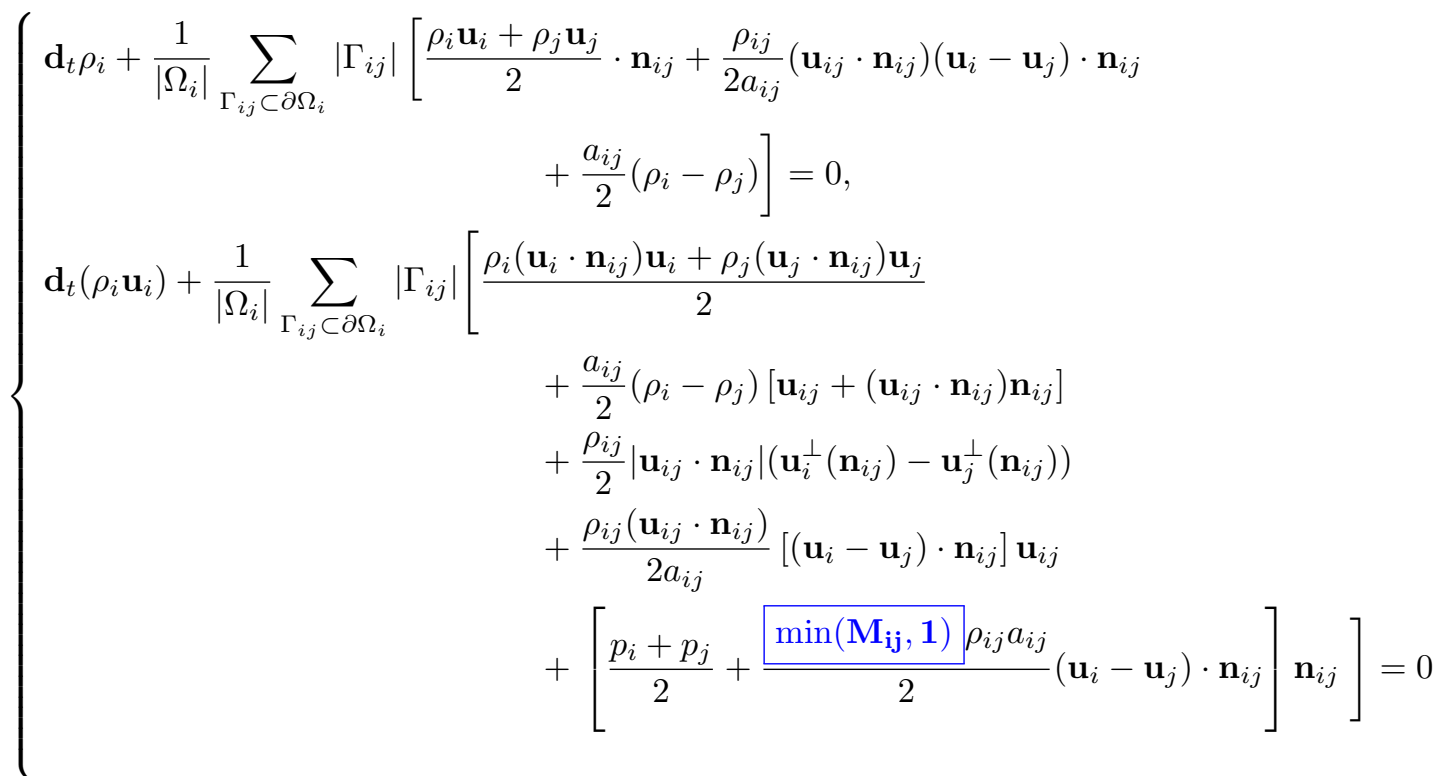

where the difference with respect to the classical Roe scheme was enlighted by a blue framebox. Then, in equation (13b), $\theta_{i j}=1$ is replaced by $\theta_{i j}=\min \left(M_{i j}, 1\right)$ and in $(15), \theta_{i j}=1$ is replaced by $\theta_{i j}=M \tilde{M}_{i j}$. In equation (16), $\delta=1$ is then replaced by $\delta=0$. Accuracy of this low Mach number fix will be numerically studied in the last section.

The explicit Roe scheme corrected with the Dellacherie et al. fix is stable at low Mach number under a standard CFL stability criterion $\Delta t=\mathcal{O}(M)$. 


\subsubsection{The fix of Rieper [47]}

The low Mach correction of [47] consists in replacing all occurrence of the term $\Delta(\mathbf{u} \cdot \mathbf{n})$ in the Roe scheme by $\min \left(M_{i j}, 1\right) \Delta(\mathbf{u} \cdot \mathbf{n})$, where $M_{i j}$ is of the order of the Mach number computed at each edge of the mesh. More precisely, the scheme reads in the subsonic case (13)

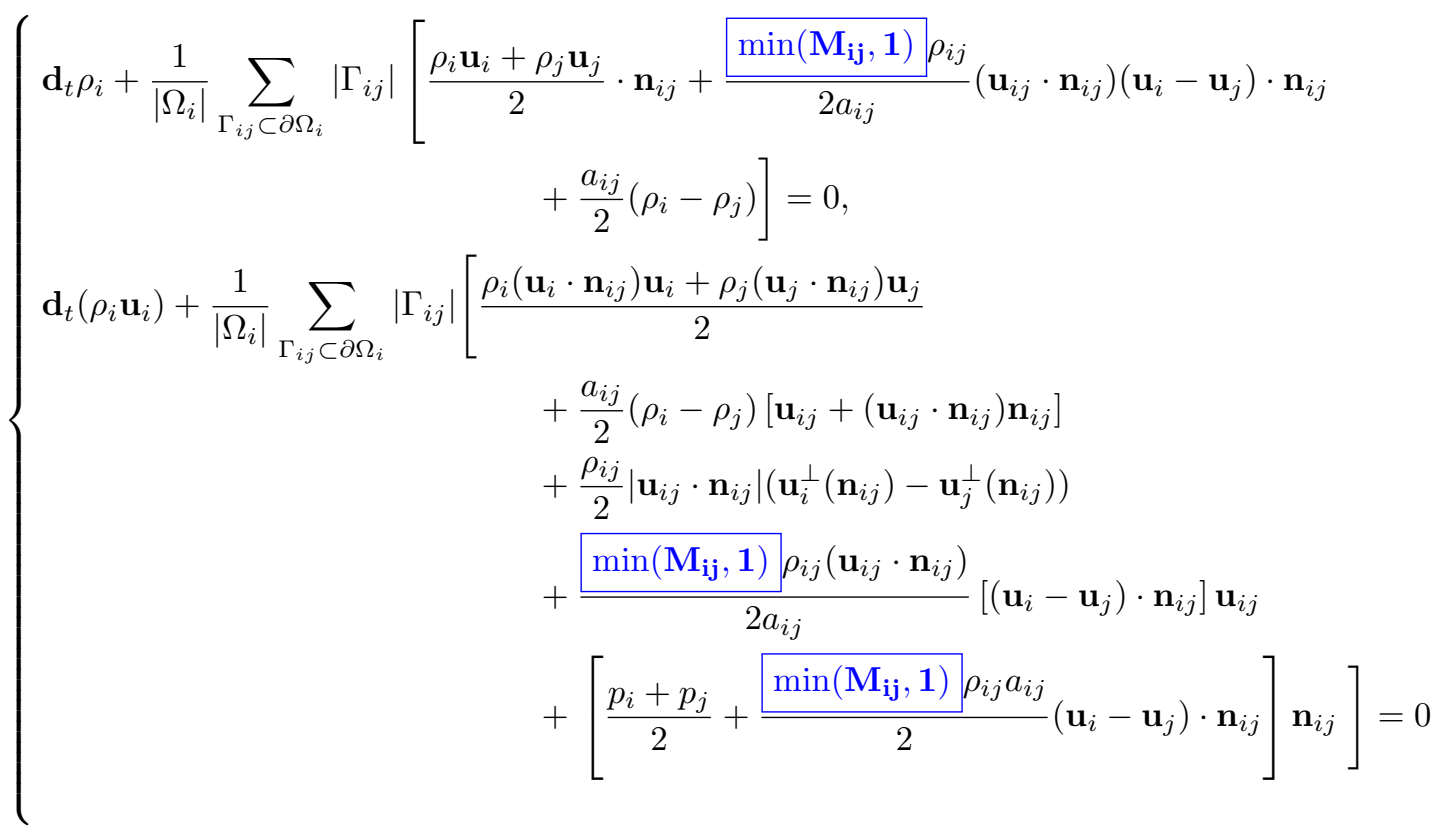

The impact of the correction of Rieper on $\left(\tilde{p}^{(1)}, \tilde{\mathbf{u}}^{(0)}\right)_{i}$ is the same as the one of Dellacherie et al. . Accuracy of this low Mach number fix will be also numerically studied in the last section.

The explicit Roe scheme corrected with the Rieper fix is also stable at low Mach number under a standard CFL stability criterion $\Delta t=\mathcal{O}(M)$.

\subsubsection{The Roe-Turkel scheme [23]}

The fix of Guillard and Viozat [23] consists in preconditioning the diffusion matrix of the Roe scheme. The diffusive part of the Roe scheme (13) is modified a lot with the Roe-Turkel scheme when the flow is subsonic. Indeed, in the subsonic case, the Roe-Turkel scheme is given by

$$
\left\{\begin{array}{c}
\mathbf{d}_{t} \rho_{i}+\frac{1}{\left|\Omega_{i}\right|} \sum_{\Gamma_{i j} \subset \partial \Omega_{i}}\left|\Gamma_{i j}\right|\left[\frac{\rho_{i} \mathbf{u}_{i}+\rho_{j} \mathbf{u}_{j}}{2} \cdot \mathbf{n}_{i j}+\frac{d_{1}}{2} \rho_{i j}\left(\mathbf{u}_{i}-\mathbf{u}_{j}\right) \cdot \mathbf{n}_{i j}+\frac{d_{2}}{2}\left(\rho_{i}-\rho_{j}\right)\right]=0 \\
\mathbf{d}_{t}\left(\rho_{i} \mathbf{u}_{i}\right)+\frac{1}{\left|\Omega_{i}\right|} \sum_{\Gamma_{i j} \subset \partial \Omega_{i}}\left|\Gamma_{i j}\right|\left[\frac{\rho_{i}\left(\mathbf{u}_{i} \cdot \mathbf{n}_{i j}\right) \mathbf{u}_{i}+\rho_{j}\left(\mathbf{u}_{j} \cdot \mathbf{n}_{i j}\right) \mathbf{u}_{j}}{2}+\frac{p_{i}+p_{j}}{2} \mathbf{n}_{i j}\right. \\
+\frac{\rho_{i j}}{2}\left|\mathbf{u}_{i j} \cdot \mathbf{n}_{i j}\right|\left(\mathbf{u}_{i}^{\perp}\left(\mathbf{n}_{i j}\right)-\mathbf{u}_{j}^{\perp}\left(\mathbf{n}_{i j}\right)\right) \\
\left.+\frac{\mathbf{d}_{3}}{2} \rho_{i j}\left(\mathbf{u}_{i}-\mathbf{u}_{j}\right) \cdot \mathbf{n}_{i j}+\frac{\mathbf{d}_{4}}{2}\left(\rho_{i}-\rho_{j}\right)\right]=0
\end{array}\right.
$$

where

$$
\begin{array}{ll}
d_{1}=\frac{\mathbf{u}_{i j} \cdot \mathbf{n}_{i j}}{\sqrt{X}}\left(1+\beta^{2}\right), & d_{2}=\frac{\left(\mathbf{u}_{i j} \cdot \mathbf{n}_{i j}\right)^{2}}{\sqrt{X}}\left(\beta^{2}-1+\frac{2 a_{i j}^{2}}{\left(\mathbf{u}_{i j} \cdot \mathbf{n}_{i j}\right)^{2}}\right), \\
\mathbf{d}_{3}=d_{1} \mathbf{u}_{i j}+d_{9} \mathbf{n}_{i j}, & \mathbf{d}_{4}=d_{2} \mathbf{u}_{i j}-\frac{s \times r}{\beta^{2}} d_{1} \mathbf{n}_{i j}, \\
d_{9}=\frac{\left(\mathbf{u}_{i j} \cdot \mathbf{n}_{i j}\right)^{2}}{\sqrt{X}}\left(1-\beta^{2}+\frac{2 \beta^{2} a_{i j}^{2}}{\left(\mathbf{u}_{i j} \cdot \mathbf{n}_{i j}\right)^{2}}\right)
\end{array}
$$

with $X=\left[\left(1-\beta^{2}\right) \mathbf{u}_{i j} \cdot \mathbf{n}_{i j}\right]^{2}+4 \beta^{2} a_{i j}^{2}, s=\frac{1}{2}\left(\mathbf{u}_{i j} \cdot \mathbf{n}_{i j}-\sqrt{X}\right)-\mathbf{u}_{i j} \cdot \mathbf{n}_{i j} \beta^{2}$ and $r=\frac{1}{2}\left(\mathbf{u}_{i j} \cdot \mathbf{n}_{i j}+\right.$ $\sqrt{X})-\mathbf{u}_{i j} \cdot \mathbf{n}_{i j} \beta^{2}$. Now, the dimensionless version (18) as in [23] is given. The parameter $\beta$ is chosen of the order of the Mach number, we take $\beta=M \tilde{\beta}$ where $\tilde{\beta}$ is a parameter of order one. We remark that $X$ can be written as

$$
X=u_{0}^{2} Y \quad \text { where } \quad Y=\left(\tilde{\mathbf{u}}_{i j} \cdot \mathbf{n}_{i j}\right)^{2}\left(1-M^{2} \tilde{\beta}^{2}\right)^{2}+4 \tilde{\beta}^{2} \tilde{a}_{i j}^{2}
$$


and $Y$ is a variable of order one. The dimensionless Roe-Turkel scheme is then given by

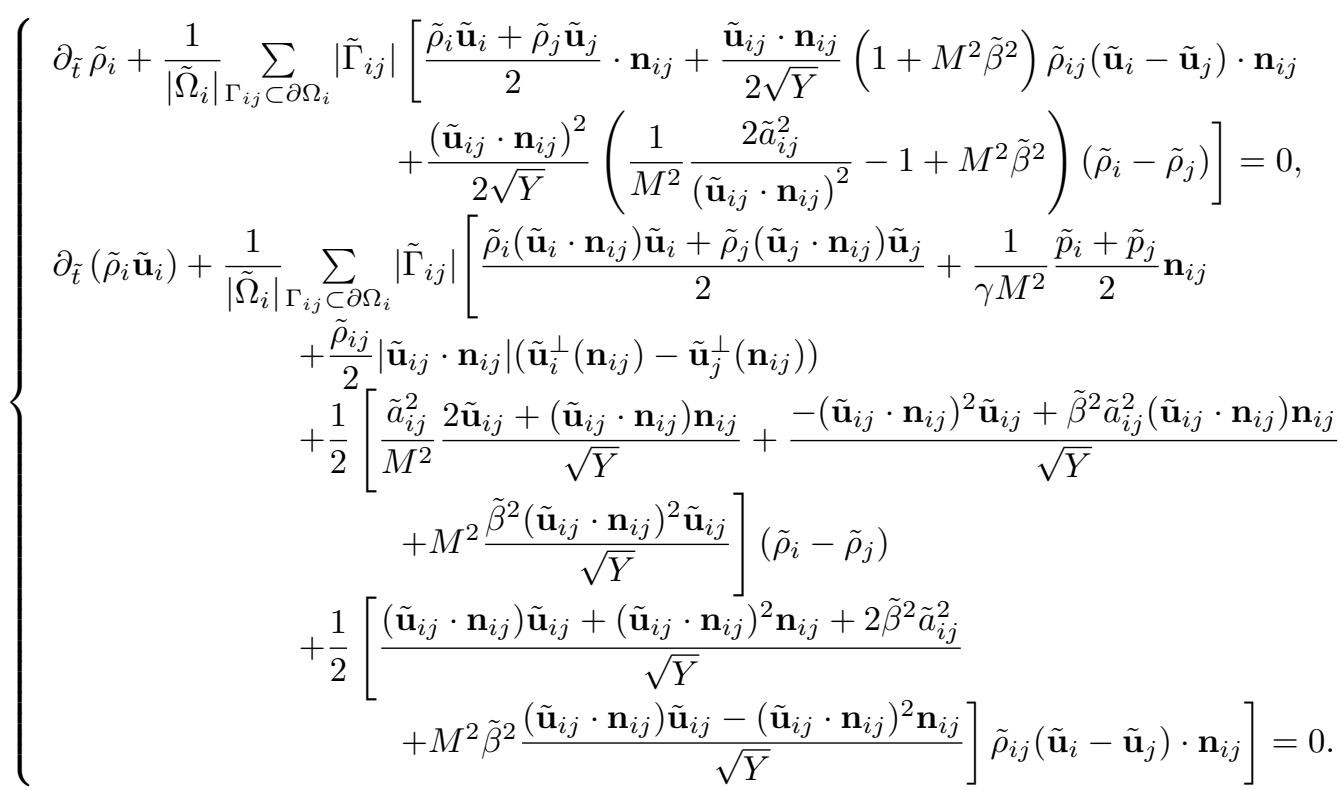

Then, following [23], it can be proved that $\tilde{\rho}^{(0)}$ is uniform, and that if $\tilde{\rho}^{(1)}$ is uniform and constant at inlet and outlet boundaries (equal to 0 ), then $\tilde{\rho}^{(1)}$ is uniformly 0 . Finally, $\tilde{p}-\tilde{p}^{(0)}=\mathcal{O}\left(M^{2}\right)$ and there is no accuracy problem at low Mach number with the Roe-Turkel scheme.

As noted in [5, 11], the explicit Roe-Turkel scheme is stable at low Mach number under a more restrictive CFL stability criterion $\Delta t=\mathcal{O}\left(M^{2}\right)$. It practically prevents the use of a time explicit integration for multidimensional computations. An other restriction of this scheme is that we numerically remark instabilities if the parameter $\beta$ is chosen locally at each mesh interface. However, the scheme seems to be stable if the parameter $\beta$ is chosen over the whole domain. This choice excludes the computation of a multi-regime flow combining low and huge Mach number regions. This stability problem induced by a local definition of $\beta$ could be solved by using the recent scheme proposed by Bouchut et al. in [6], in which a low Mach accurate scheme was proposed, which is also entropic (and therefore more stable). The extension of [23] to all Mach number, and especially trying to circumvent the dependency on a reference Mach number was the topic of several articles, see [32] for a review. The common point to all these schemes is that they degenerate towards [23] when the Mach number goes to 0. As we are focused in this article only on low Mach number behaviour, we only deal with the solver proposed [23], as its all Mach number extensions have the same low Mach number behaviour. All these schemes suffer from the same CFL restriction as the Roe-Turkel scheme.

In this section, the accuracy problem of the Roe scheme in the low Mach number limit has been recalled. Some previously proposed fixes have been also recalled. The next section is dedicated to the behavior of these different schemes for the computation of an acoustic wave propagating in a low Mach number flow.

\section{Impact of the low Mach number corrections on the com- putation of an acoustic wave in a low Mach number flow}

In this section, we are interested in the accurate computation of an acoustic wave in a low Mach number flow. In subsection 2.1, the equations of acoustic waves in a low Mach number flow are derived. Then, in subsection 2.2, the selected test case is described. Numerical results obtained with different finite volumes schemes, with and without low Mach number fixes are shown, and proved to be either unable to propagate acoustic waves or to give a wrong order of accuracy. In order to explain the numerical results obtained, a similar approach as in section 1 is followed in subsection 2.3: an asymptotic expansion of the discrete equations is performed, and compared with the continuous system derived in subsection 2.1. This asymptotic expansion is done with and without low Mach number fix for explaining the results obtained in subsection 2.2. 


\subsection{Two-scale dimensionless equations: acoustic limit on an incompress- ible flow}

In this section, we are interested in deriving a system which allows to separate the material time scale information (moving at velocity $\mathbf{u} \cdot \mathbf{n}$ ) from the acoustic time scale which corresponds to phenomena that move approximately at the velocity of the sound $a_{0}$ when the Mach number is sufficiently low. It means that we have now two reference time scales $T_{\text {mat }}=x_{0} / u_{0}=t_{0}$ and $T_{a c}=x_{0} / a_{0}$ yielding two dimensionless time scales, namely

$$
\tilde{t}=\frac{t}{T_{\text {mat }}} \quad \text { and } \quad \tau=\frac{t}{T_{a c}}=\frac{t}{x_{0} / a_{0}}=\frac{t}{u_{0} \times T_{\text {mat }} / a_{0}}=\frac{t}{M \times T_{\text {mat }}}=\frac{\tilde{t}}{M} .
$$

The material time $\tilde{t}$ and the acoustic time $\tau$ have a ratio of order $M$. This justifies to split the time dependency of the variables into two times: $\tilde{t}$ but also $\tau$. Details on this development can be found in [37]. Then $\varphi \in\{\rho, \mathbf{u}\}$ can be written as an expansion in exponent of the Mach number $M$ :

$$
\tilde{\varphi}(\tilde{\mathbf{x}}, \tilde{t} ; M)=\sum_{n=0}^{N} M^{n} \tilde{\varphi}^{(n)}(\tilde{\mathbf{x}}, \tilde{t}, \tau)+\mathcal{O}\left(M^{N+1}\right)
$$

with $\tau=\tilde{t} / M$. Then the derivative with respect to the time is

$$
\partial_{\tilde{t}} \tilde{\varphi}(\tilde{\mathbf{x}}, \tilde{t} ; M)=\sum_{n=0}^{N} M^{n}\left(\partial_{\tilde{t}} \tilde{\varphi}^{(n)}(\tilde{\mathbf{x}}, \tilde{t}, \tau)+\frac{1}{M} \partial_{\tau} \tilde{\varphi}^{(n)}(\tilde{\mathbf{x}}, \tilde{t}, \tau)\right)+\mathcal{O}\left(M^{N+1}\right) .
$$

By injecting the development $(22)$ in (5), the momentum equation at order $M^{-2}$ gives $\nabla_{\tilde{\mathbf{x}}} \tilde{p}^{(0)}=0$ and then, since $p$ is a regular function of $\rho$,

$$
\nabla_{\tilde{\mathbf{x}}} \tilde{\rho}^{(0)}=0 .
$$

At order $M^{-1}$, we obtain

$$
\left\{\begin{array}{l}
\partial_{\tau} \tilde{\rho}^{(0)}=0 \\
\partial_{\tau}\left(\tilde{\rho}^{(0)} \tilde{\mathbf{u}}^{(0)}\right)+\frac{1}{\gamma} \nabla_{\tilde{\mathbf{x}}} \tilde{p}^{(1)}=0
\end{array}\right.
$$

and then, using (23) and (24a), leads to

$$
\tilde{\rho}^{(0)}(\tilde{\mathbf{x}}, \tilde{t}, \tau)=\tilde{\rho}^{(0)}(\tilde{t})
$$

At order $M^{0}$, we get

$$
\left\{\begin{array}{l}
\mathbf{d}_{\tilde{t}} \tilde{\rho}^{(0)}+\partial_{\tau} \tilde{\rho}^{(1)}+\nabla_{\tilde{\mathbf{x}}} \cdot\left(\tilde{\rho}^{(0)} \tilde{\mathbf{u}}^{(0)}\right)=0, \\
\partial_{\tilde{t}}\left(\tilde{\rho}^{(0)} \tilde{\mathbf{u}}^{(0)}\right)+\partial_{\tau}(\tilde{\rho} \tilde{\mathbf{u}})^{(1)}+\nabla_{\tilde{\mathbf{x}}} \cdot\left(\tilde{\rho}^{(0)} \tilde{\mathbf{u}}^{(0)} \otimes \tilde{\mathbf{u}}^{(0)}\right)+\frac{1}{\gamma} \nabla_{\tilde{\mathbf{x}}} \tilde{p}^{(2)}=0 .
\end{array}\right.
$$

With $(24 b)$ and $(26 \mathrm{a})$, we obtain that $\left(\tilde{\rho}^{(1)}, \tilde{\rho}^{(0)} \tilde{\mathbf{u}}^{(0)}\right)$ satisfies

$$
\left\{\begin{array}{l}
\partial_{\tau} \tilde{\rho}^{(1)}+\nabla_{\tilde{\mathbf{x}}} \cdot\left(\tilde{\rho}^{(0)} \tilde{\mathbf{u}}^{(0)}\right)=-\mathbf{d}_{\tilde{t}} \tilde{\rho}^{(0)} \\
\partial_{\tau}(\tilde{\rho} \tilde{\mathbf{u}})^{(0)}+\frac{1}{\gamma} \nabla_{\tilde{\mathbf{x}}} \tilde{p}^{(1)}=0
\end{array}\right.
$$

By multiplying (27a) by $\mathbf{d}_{\tilde{\rho}} \tilde{p}\left(\tilde{\rho}^{(0)}\right)$ where $\tilde{\rho}^{(0)}=\tilde{\rho}^{(0)}(\tilde{t})$, we obtain the first order wave equation on $\left(\tilde{p}^{(1)},(\tilde{\rho} \tilde{\mathbf{u}})^{(0)}\right)$ with a source term

$$
\left\{\begin{array}{l}
\partial_{\tau} \tilde{p}^{(1)}+\mathbf{d}_{\tilde{\rho}} \tilde{p}\left(\tilde{\rho}^{(0)}\right) \nabla_{\tilde{\mathbf{x}}} \cdot(\tilde{\rho} \tilde{\mathbf{u}})^{(0)}=-\mathbf{d}_{\tilde{t}} \tilde{p}^{(0)} \\
\partial_{\tau}(\tilde{\rho} \tilde{\mathbf{u}})^{(0)}+\frac{1}{\gamma} \nabla_{\tilde{\mathbf{x}}} \tilde{p}^{(1)}=0
\end{array}\right.
$$

Indeed, since $\tilde{p}$ is a regular function of $\tilde{\rho}$, we have $\mathbf{d}_{\tilde{\rho}} \tilde{p}\left(\tilde{\rho}^{(0)}\right) \tilde{\rho}^{(1)}=\tilde{p}^{(1)}$ and $\mathbf{d}_{\tilde{\rho}} \tilde{p}\left(\tilde{\rho}^{(0)}\right) \mathbf{d}_{\tilde{t}} \tilde{\rho}^{(0)}=\mathbf{d}_{\tilde{t}} \tilde{p}^{(0)}$. 


\subsection{Acoustic wave propagation in a low Mach number flow}

\subsubsection{Test case description}

The domain $\Omega$ is equal to $[0 ; 1]$. A stationary low Mach number flow is considered, characterized by its velocity $u_{\infty}$ and density $\rho_{\infty}$. The initial condition of this test is obtained by adding to this low Mach number flow an acoustic wave which moves at the speed $u+a$. Based on the analysis of subsection 2.1, the density and the pressure perturbations are of order $\mathcal{O}(M)$, whereas the velocity is of order $\mathcal{O}(1)$. The initial density is then given by

$$
\rho_{0}(x)=\rho_{\infty}\left(1+M_{\infty} \sin (2 \pi f x)\right)
$$

and since $u-\frac{2 a}{\gamma-1}$ is a Riemann invariant of the wave associated to $x / t=u+a$, the initial velocity is taken as

$$
u_{0}(x)=u_{\infty}+\frac{2}{\gamma-1}\left(a\left(\rho_{0}(x)\right)-a_{\infty}\right)
$$

where $u_{\infty}=M_{\infty} \times a_{\infty}$. We choose $\rho_{\infty}=1, M_{\infty}=10^{-3}$ and $f=5$ then $a_{\infty}=\sqrt{\kappa \gamma}$. For the numerical application, the equation of state is given by (2) with $\kappa=1$ and $\gamma=2$. A periodic boundary condition linking the left and right boundaries is used.

Note that the expressions given by (29) and (30) are matching with the asymptotic development found in subsection 2.1. In adimensioned variables, (29) gives

$$
\tilde{\rho}_{0}(x)=1+M_{\infty} \sin (2 \pi f x)
$$

which matches with a $\mathcal{O}(M)$ perturbation, whereas (30) can be reformulated as

$$
\begin{aligned}
\tilde{u}_{0}(x) & =1+\frac{2}{(\gamma-1) u_{\infty}}\left(a\left(\rho_{0}(x)\right)-a_{\infty}\right) \\
& =1+\frac{2}{(\gamma-1) u_{\infty}}\left(\sqrt{\kappa \gamma \rho_{0}(x)^{\gamma-1}}-\sqrt{\kappa \gamma \rho_{\infty}^{\gamma-1}}\right) \\
& =1+\frac{2 a_{\infty}}{(\gamma-1) u_{\infty}}\left(\tilde{\rho}_{0}(x)^{(\gamma-1) / 2}-1\right) \\
& =1+\frac{2}{(\gamma-1) M_{\infty}}\left(\left(1+M_{\infty} \sin (2 \pi f x)\right)^{(\gamma-1) / 2}-1\right) \\
& =1+\frac{2}{(\gamma-1) M_{\infty}}\left(1+\frac{\gamma-1}{2} M_{\infty} \sin (2 \pi f x)+\mathcal{O}\left(M^{2}\right)-1\right) \\
\Rightarrow \tilde{u}_{0}(x) & =1+\sin (2 \pi f x)+\mathcal{O}(M) .
\end{aligned}
$$

An exact solution at time $t_{\max }$ can be computed with the method of characteristics. The exact solution is given by

$$
\rho_{\text {exact }}\left(x, t_{\max }\right)=\rho_{0}\left(x-\xi t_{\max }\right) \quad \text { and } \quad u_{\text {exact }}\left(x, t_{\max }\right)=u_{0}\left(x-\xi t_{\max }\right)
$$

where $\xi$ is solution of the non linear equation

$$
\xi-u_{0}\left(x-\xi t_{\max }\right)-a_{0}\left(x-\xi t_{\max }\right)=0
$$

and can be found by using Newton's method.

All the numerical results presented below are obtained with a regular mesh $\Delta x=1 / N$. Implicit and explicit time stepping will be used. For implicit time stepping, a constant time step will be used while for explicit time stepping, the time step $(\Delta t)_{n}$ satisfies

$$
(\Delta t)_{n}=\mathrm{CFL} \times \frac{\Delta x}{\max _{i}\left(\left|u_{i}^{n}\right|+c_{i}^{n}\right)} .
$$

The CFL number will be specified in each case.

Next section is dedicated to the numerical results obtained on this test case.

\subsubsection{Numerical results with the Roe scheme and with some low Mach number fixes}

In Figure 1, results obtained on this test case with the Roe-Turkel scheme are shown. For a time of the order of the Mach number, the density obtained with the Roe-Turkel scheme is far from the exact solution. Indeed, a very fast damping of the wave amplitude of the density is observed. For 

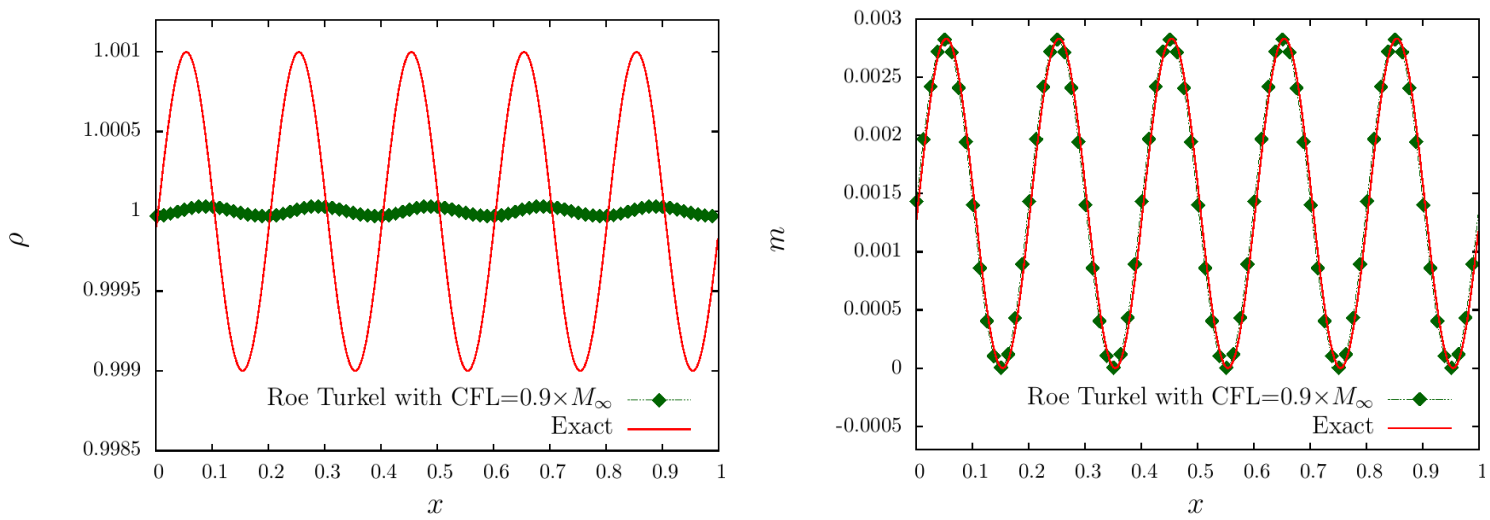

Figure 1: Density $\rho$ (left) and momentum $m$ (right) obtained at time $t=0.003 \mathrm{~s}$ on the sinusoidal test case described in subsubsection 2.2.1 with $N=400$ regular cells, $M_{\infty}=10^{-3}$ and a first order approximation (explicit time stepping).

being more explicit, the damping of the acoustic wave with the Roe and the Roe-Turkel schemes are compared. We define the adimensioned amplitude of the wave as

$$
A_{a d}(t)=\frac{\max _{x \in[0,1]} \rho(x, t)-\min _{x \in[0,1]} \rho(x, t)}{2\left(\max _{x \in[0,1]} \rho(x, 0)-\min _{x \in[0,1]} \rho(x, 0)\right)} .
$$

The damping of the amplitude is studied for the Mach number $M_{\infty}$ equal to $1,10^{-1}, 10^{-2}, 10^{-3}$, with both explicit and implicit time stepping for rejecting any influence of the time integration. For the implicit time stepping, the time step was chosen equal to 0.0001 for all the Mach numbers. For the explicit time stepping, the time step was chosen equal to i) $10^{-4}$ for the Mach number equal to 1 and $10^{-1}$, ii) $10^{-5}$ for the Mach number equal to $10^{-2}$ and iii) $10^{-6}$ for the Mach number equal to $10^{-3}$. In Figure 2, the dimensionless amplitude is drawn for a Mach number equal to $1,10^{-1}$, $10^{-2}, 10^{-3}$ for the Roe and Roe-Turkel schemes, for $t \in[0,0.003]$. With the Roe-Turkel scheme, for both the explicit and implicit time stepping, the lower the Mach number is, the faster the amplitude is going to 0 . With the Roe scheme, the dimensionless amplitude stays close to 1 , and is nearly Mach independent. This damping of the acoustic wave on the density will be explained in subsubsection 2.3.1.

Results obtained with an explicit time stepping with the Dellacherie et al. and Rieper fixes are shown in Figure 3, and compared with the results obtained with the Roe scheme. Numerical results show that the numerical scheme with the different fixes is stable only with a degraded CFL number, approximately half of the CFL number of the original Roe scheme. The numerical error is computed on the density and on the momentum, still with these numerical fluxes, for a first order finite volume computation, and also with a second order approximation. The second order is achieved with a discontinuous Galerkin with piecewise linear approximation in space, and a SSP integration in time [9]. Some details are given on this method here. We denote by $V^{k}$ the finite element space approximation composed of piecewise polynomial functions of degree lower or equal to $k$ on the mesh (polynomial on the cells of the mesh, discontinuous on sides). We denote by $V\left(\Omega_{i}, k\right)$ the space of polynoms of degree lower or equal to $k$ in the cell $\Omega$ of the mesh. Then the spatial discretisation of (3) is: for all $\Omega_{i}$, find $\mathcal{U}_{h} \in V^{k}$ such that

$$
\forall \varphi \in V\left(\Omega_{i}, k\right) \quad \int_{\Omega_{i}} \varphi \partial_{t} \mathcal{U}_{h}-\int_{\Omega_{i}} \mathbf{f}\left(\mathcal{U}_{h}\right) \nabla \varphi+\sum_{\Gamma_{i j} \subset \Omega_{i}} \int_{\partial K} \Phi_{i j}\left(\mathcal{U}_{h}, \mathbf{n}_{i j}\right) \varphi=0 .
$$

For a $k$ th order approximation space, the optimal order is $k+1$. Note that the discontinuous Galerkin method includes finite volumes numerical fluxes on sides, for stabilization. Also, the discontinuous Galerkin method exactly matches with the first order finite volumes method when $k=0$. From a practical point of view, the spatial integrals are computed with a $2 k$ th order accurate quadrature formula on the cells, and a $(2 k+1)$ th order accurate quadrature formula on the sides. The time integration is achieved with a SSP explicit scheme of order $k+1$ for a $k$ th approximation order by cell [19]. As the flow is regular, no additional limiting procedure is added. Results are shown in Figure 4. The coarser and finer meshes contain $N=200$ and $N=6400$ regular cells respectively, and $\Delta x=1 / N$. As expected, the Roe-Turkel finite volume approximation does not converge. The right order of accuracy is observed with the finite volume approximation for the 

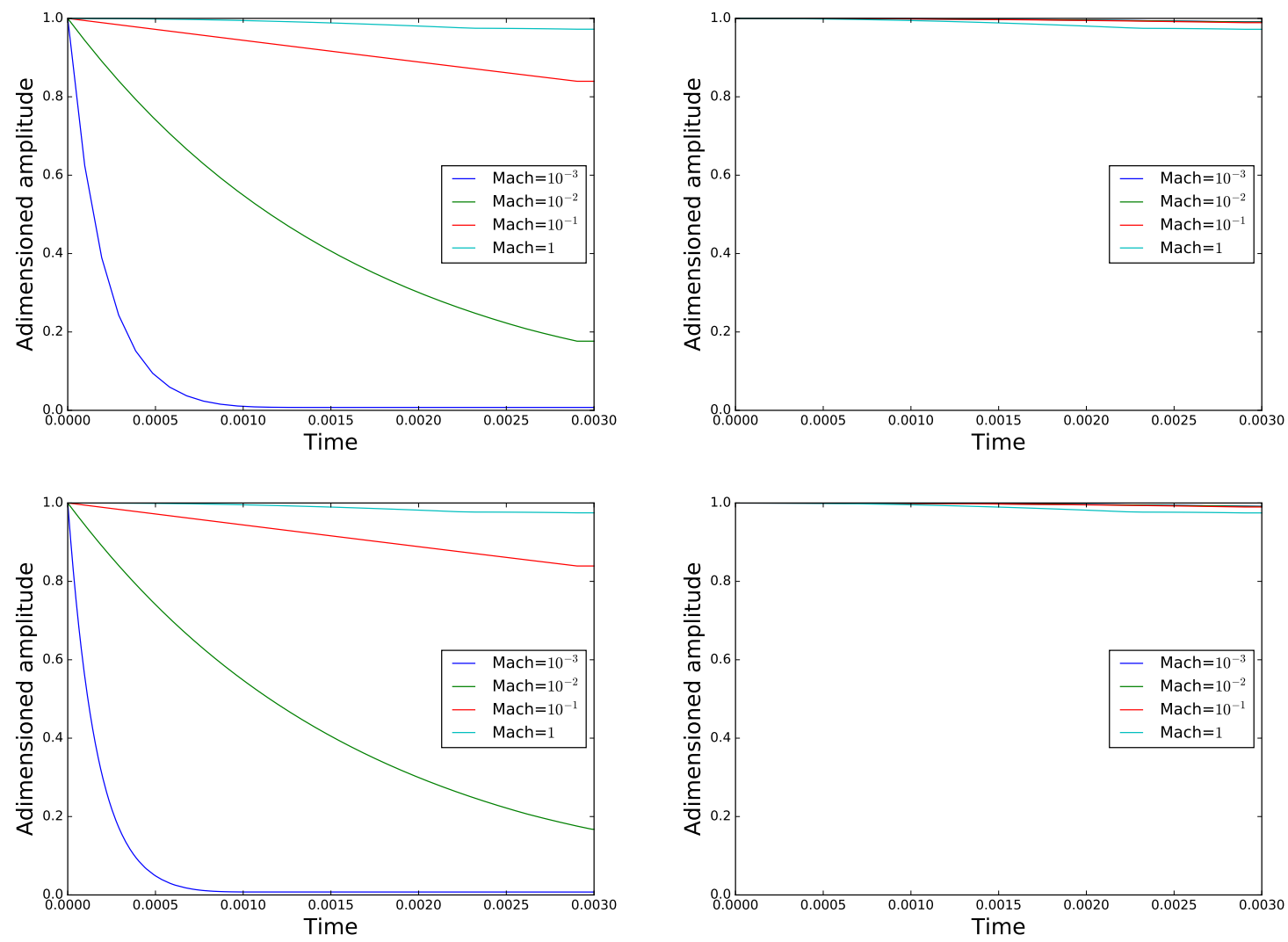

Figure 2: Damping of the density component on the sinusoidal test case described in subsubsection 2.2.1, with implicit time stepping (top) and explicit time stepping (bottom), for the Roe-Turkel scheme (left) and the Roe scheme (right), for the Mach number $M_{\infty}$ equal to $1,10^{-1}, 10^{-2}, 10^{-3}$.

other fixes. With the second order scheme, the right order is observed on the density, but not on the momentum, for which only a first order of accuracy is observed for the Roe scheme corrected with Dellacherie et al. or Rieper fixes.

In this section, numerical tests with the low Mach number fixes were performed on the test of subsubsection 2.2.1. These tests reveal that for the computation of an acoustic wave in a low Mach number flow

- the Roe-Turkel scheme is not accurate,

- the Dellacherie et al. and Rieper fixes are accurate only at first order, and with a degraded CFL stability criterion.

The next section aims at explaining these results.

\subsection{Analysis}

The aim of this section is to analyze the problems evidenced by the numerical results of subsubsection 2.2.2, namely the fact that the Roe-Turkel scheme does not propagate acoustics, and the fact that fixes of $[47,12]$ have a reduced CFL compared with the Roe scheme and give the wrong order of accuracy on the momentum 

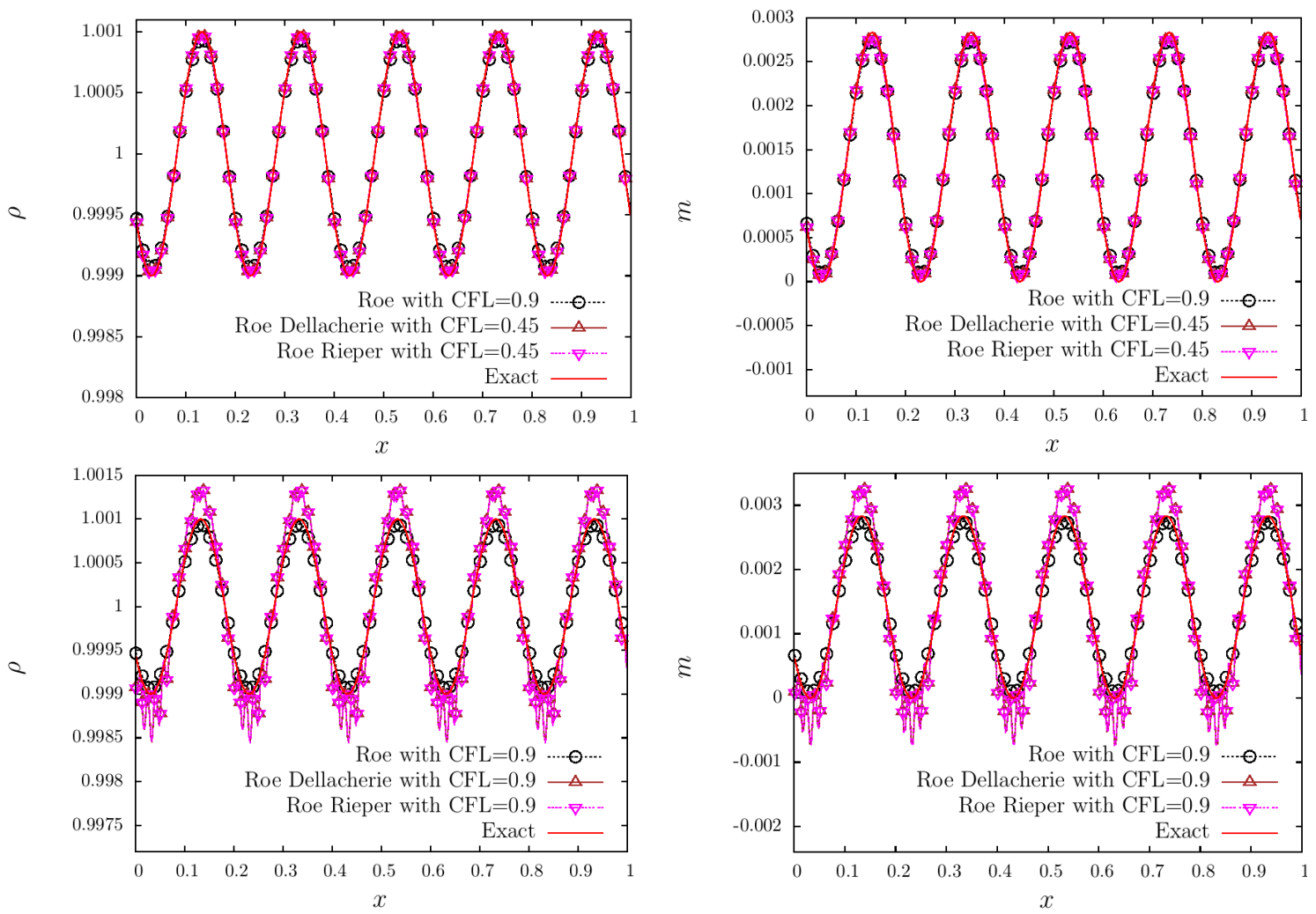

Figure 3: Density $\rho$ (left) and momentum $m$ (right) obtained at time $t=0.3408 \mathrm{~s}$ on the sinusoidal test case described in subsubsection 2.2.1 with $N=400$ regular cells, $M_{\infty}=10^{-3}$ and a first order approximation (explicit time stepping) with a CFL number of 0.9 (bottom) and a CFL number of 0.45 (top).
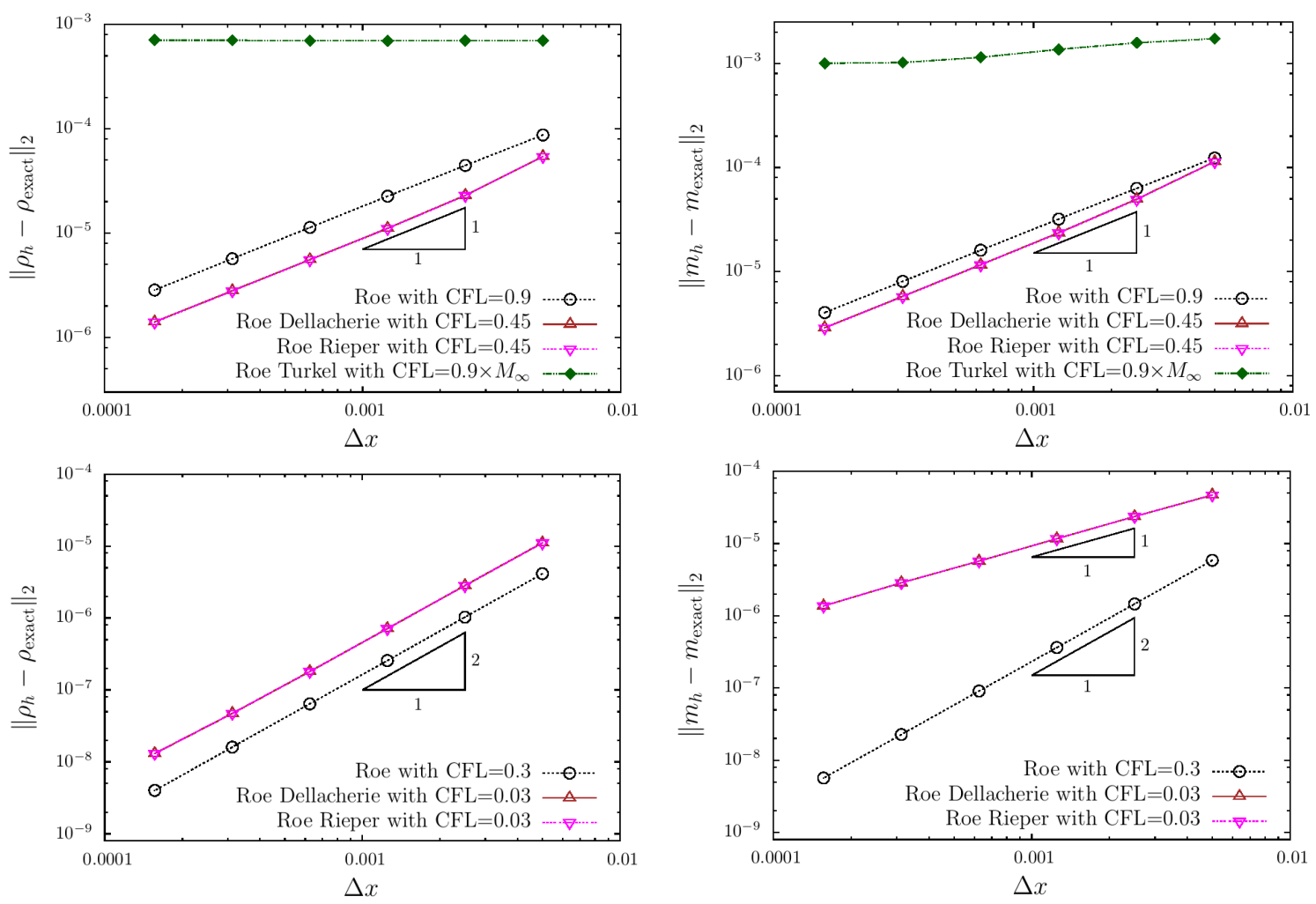

Figure 4: Order obtained on the density (left) and on the momentum (right) on the sinusoidal test case described in subsubsection 2.2.1 with $M_{\infty}=10^{-3}$ at time $t=0.3408 \mathrm{~s}$ with the Roe scheme and different fixes with a first (top) and a second order (bottom) approximations. 


\subsubsection{Two scales dimensionless study of the Roe-Turkel scheme}

We consider the variables (4), (14) and (20). The dimensionless version of the scheme (13) is given by

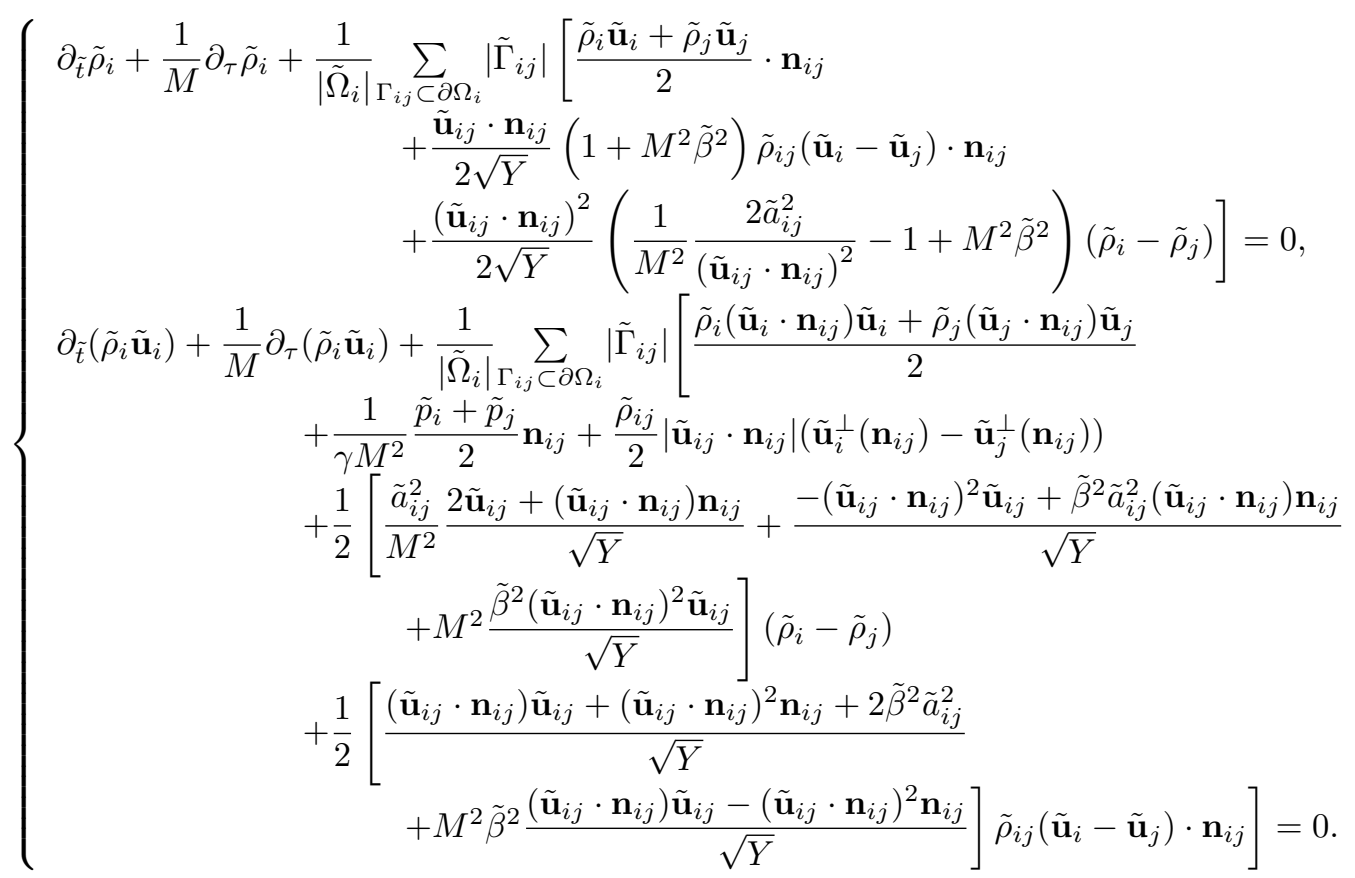

Proposition 2.1. If the scales in $\mathcal{O}\left(M^{k}\right)$ are supposed to be separated, and if we suppose that the boundary conditions are either wall or periodic, then (31) implies

$$
\forall i, \quad \tilde{\rho}_{i}^{(0)}=\tilde{\rho}^{(0)}
$$

and

$$
\forall i, \quad \tilde{\rho}_{i}^{(1)}=\tilde{\rho}^{(1)} .
$$

Proof. Using the development (21) in the first equation of (31), we obtain at order $M^{-2}$

$$
\sum_{\Gamma_{i j} \subset \partial \Omega_{i}}\left|\tilde{\Gamma}_{i j}\right| \frac{\tilde{a}_{i j}^{(0)}}{\sqrt{Y}}\left(\tilde{\rho}_{i}^{(0)}-\tilde{\rho}_{j}^{(0)}\right)=0 .
$$

By multiplying by $\tilde{\rho}_{i}^{(0)}$ and by summing over all cells, it gives (with periodic boundary condition or wall boundary condition)

$$
\begin{aligned}
& \sum_{i} \sum_{\Gamma_{i j} \subset \partial \Omega_{i}}\left|\tilde{\Gamma}_{i j}\right| \frac{\tilde{a}_{i j}^{(0)}}{\sqrt{Y}} \tilde{\rho}_{i}^{(0)}\left(\tilde{\rho}_{i}^{(0)}-\tilde{\rho}_{j}^{(0)}\right)=0 \\
\Rightarrow & \sum_{\Gamma_{i j} \subset \AA}\left|\tilde{\Gamma}_{i j}\right|\left[\frac{\tilde{a}_{i j}^{(0)}}{\sqrt{Y}} \tilde{\rho}_{i}^{(0)}\left(\tilde{\rho}_{i}^{(0)}-\tilde{\rho}_{j}^{(0)}\right)+\frac{\tilde{a}_{j i}^{(0)}}{\sqrt{Y}} \tilde{\rho}_{j}^{(0)}\left(\tilde{\rho}_{j}^{(0)}-\tilde{\rho}_{i}^{(0)}\right)\right]=0 \\
\Rightarrow & \sum_{\Gamma_{i j} \subset \AA}\left|\tilde{\Gamma}_{i j}\right| \frac{\tilde{a}_{i j}^{(0)}}{\sqrt{Y}}\left(\tilde{\rho}_{i}^{(0)}-\tilde{\rho}_{j}^{(0)}\right)^{2}=0
\end{aligned}
$$

where $\stackrel{\circ}{\Omega}=\Omega \backslash \partial \Omega$ is the interior of $\Omega$ and we get

$$
\forall i, \quad \tilde{\rho}_{i}^{(0)}=\tilde{\rho}^{(0)} .
$$

Then, the order $M^{-1}$ gives

$$
\partial_{\tau} \tilde{\rho}^{(0)}+\frac{1}{\left|\tilde{\Omega}_{i}\right|} \sum_{\Gamma_{i j} \subset \partial \Omega_{i}}\left|\tilde{\Gamma}_{i j}\right| \frac{\tilde{a}_{i j}^{(0)}}{\sqrt{Y}}\left(\tilde{\rho}_{i}^{(1)}-\tilde{\rho}_{j}^{(1)}\right)=0 .
$$


By summing over all cells, we get $\partial_{\tau} \tilde{\rho}^{(0)}=0$ and then since we get the same equation for $\tilde{\rho}_{i}^{(1)}$ as for $\tilde{\rho}_{i}^{(0)}$, we have

which ends the proof.

$$
\forall i, \quad \tilde{\rho}_{i}^{(1)}=\tilde{\rho}^{(1)}
$$

Thus, with the Roe-Turkel scheme, $\tilde{\rho}^{(1)}$ is uniform, which explains the strong damping observed on the density in Figure 1 and Figure 2. Therefore, the Roe-Turkel scheme cannot be accurate for the computation of a propagating acoustic waves in a low Mach number flow.

\subsubsection{Two-scale dimensionless study of the Roe scheme and the Roe scheme corrected with the Dellacherie et al. or Rieper fixes}

We consider variables (4), (14) and (20). The dimensionless version of the scheme (13) is given by

$$
\left\{\begin{aligned}
\partial_{\tilde{t}} \tilde{\rho}_{i}+\frac{1}{M} \partial_{\tau} \tilde{\rho}_{i}+\frac{1}{\left|\tilde{\Omega}_{i}\right|} \sum_{\Gamma_{i j} \subset \partial \Omega_{i}}\left|\tilde{\Gamma}_{i j}\right|\left[\frac{\tilde{\rho}_{i} \tilde{\mathbf{u}}_{i}+\tilde{\rho}_{j} \tilde{\mathbf{u}}_{j}}{2} \cdot \mathbf{n}_{i j}\right. \\
\left.+\eta_{i j} M \frac{\tilde{\rho}_{i j}}{2 \tilde{a}_{i j}}\left(\tilde{\mathbf{u}}_{i j} \cdot \mathbf{n}_{i j}\right)\left(\tilde{\mathbf{u}}_{i}-\tilde{\mathbf{u}}_{j}\right) \cdot \mathbf{n}_{i j}+\frac{\tilde{a}_{i j}}{2 M}\left(\tilde{\rho}_{i}-\tilde{\rho}_{j}\right)\right]=0 \\
\partial_{\tilde{t}}\left(\tilde{\rho}_{i} \tilde{\mathbf{u}}_{i}\right)+\frac{1}{M} \partial_{\tau}\left(\tilde{\rho}_{i} \tilde{\mathbf{u}}_{i}\right)+\frac{1}{\left|\tilde{\Omega}_{i}\right|} \sum_{\Gamma_{i j} \subset \partial \Omega_{i}}\left|\tilde{\Gamma}_{i j}\right|\left[\frac{\tilde{\rho}_{i}\left(\tilde{\mathbf{u}}_{i} \cdot \mathbf{n}_{i j}\right) \tilde{\mathbf{u}}_{i}+\tilde{\rho}_{j}\left(\tilde{\mathbf{u}}_{j} \cdot \mathbf{n}_{i j}\right) \tilde{\mathbf{u}}_{j}}{2}\right. \\
+\frac{\tilde{a}_{i j}}{2 M}\left(\tilde{\rho}_{i}-\tilde{\rho}_{j}\right)\left[\tilde{\mathbf{u}}_{i j}+\left(\tilde{\mathbf{u}}_{i j} \cdot \mathbf{n}_{i j}\right) \mathbf{n}_{i j}\right] \\
+\frac{\tilde{\rho}_{i j}}{2}\left|\tilde{\mathbf{u}}_{i j} \cdot \mathbf{n}_{i j}\right|\left(\tilde{\mathbf{u}}_{i}^{\perp}\left(\mathbf{n}_{i j}\right)-\tilde{\mathbf{u}}_{j}^{\perp}\left(\mathbf{n}_{i j}\right)\right) \\
+M \frac{\tilde{\rho}_{i j}\left(\tilde{\mathbf{u}}_{i j} \cdot \mathbf{n}_{i j}\right)}{2 \tilde{a}_{i j}}\left[\left(\tilde{\mathbf{u}}_{i}-\tilde{\mathbf{u}}_{j}\right) \cdot \mathbf{n}_{i j}\right] \tilde{\mathbf{u}}_{i j} \\
\left.+\left[\frac{1}{\gamma M^{2}} \frac{\tilde{p}_{i}+\tilde{p}_{j}}{2}+\frac{\theta_{i j}}{2 M} \tilde{\rho}_{i j} \tilde{a}_{i j}\left(\tilde{\mathbf{u}}_{i}-\tilde{\mathbf{u}}_{j}\right) \cdot \mathbf{n}_{i j}\right] \mathbf{n}_{i j}\right]=0
\end{aligned}\right.
$$

with $\left(\theta_{i j}, \eta_{i j}\right)=(1,1)$ for the Roe scheme, $\left(\theta_{i j}, \eta_{i j}\right)=\left(\varphi\left(M_{i j}\right), 1\right)$ for the Dellacherie et al. fix, $\left(\theta_{i j}, \eta_{i j}\right)=\left(\varphi\left(M_{i j}\right), \varphi\left(M_{i j}\right)\right)$ for the Rieper fix and where the function $\varphi$ is defined by $\varphi\left(M_{i j}\right):=$ $\min \left(M_{i j}, 1\right)$ with $M_{i j}=\max \left(M_{i}, M_{j}\right)=\max \left(\left\|\mathbf{u}_{i}\right\| / a_{i},\left\|\mathbf{u}_{j}\right\| / a_{j}\right)=M \max \left(\left\|\tilde{\mathbf{u}}_{i}\right\| / \tilde{a}_{i},\left\|\tilde{\mathbf{u}}_{j}\right\| / \tilde{a}_{j}\right)$.

The study of the semi-discrete scheme is organized by following the same steps as in subsection 2.1 for the continuous case.

By injecting (21) in (32), we obtain at order $M^{-1}$

$$
\left\{\begin{aligned}
\partial_{\tau} \tilde{\rho}_{i}^{(0)}+\frac{1}{2\left|\tilde{\Omega}_{i}\right|} \sum_{\Gamma_{i j} \subset \partial \Omega_{i}}\left|\tilde{\Gamma}_{i j}\right| \tilde{a}_{i j}^{(0)}\left(\tilde{\rho}_{i}^{(0)}-\tilde{\rho}_{j}^{(0)}\right)=0 \\
\partial_{\tau}\left(\tilde{\rho}_{i} \tilde{\mathbf{u}}_{i}\right)^{(0)}+\frac{1}{2\left|\tilde{\Omega}_{i}\right|} \sum_{\Gamma_{i j} \subset \partial \Omega_{i}}\left|\tilde{\Gamma}_{i j}\right|\left[\tilde{a}_{i j}^{(0)}\left(\tilde{\rho}_{i}^{(0)}-\tilde{\rho}_{j}^{(0)}\right)\left[\tilde{\mathbf{u}}_{i j}^{(0)}+\left(\tilde{\mathbf{u}}_{i j}^{(0)} \cdot \mathbf{n}_{i j}\right) \mathbf{n}_{i j}\right]\right. \\
\left.+\left[\frac{1}{\gamma}\left(\tilde{p}_{i}^{(1)}+\tilde{p}_{j}^{(1)}\right)+\delta \tilde{\rho}_{i j}^{(0)} \tilde{a}_{i j}^{(0)}\left(\tilde{\mathbf{u}}_{i}^{(0)}-\tilde{\mathbf{u}}_{j}^{(0)}\right) \cdot \mathbf{n}_{i j}\right] \mathbf{n}_{i j}\right]=0
\end{aligned}\right.
$$

with $\delta=1$ for the Roe scheme and $\delta=0$ for the Dellacherie et al. and Rieper fixes.

The following proposition is the equivalent of (25) for the semi-discrete scheme.

Proposition 2.2 (Uniformity of $\left.\tilde{\rho}^{(0)}\right)$. If at $\tau=0, \tilde{\rho}^{(0)}$ is uniform (equal to $\tilde{\rho}_{0}^{(0)}$ ) and if $\tilde{\rho}^{(0)}$ is constant at the boundaries, then

$$
\forall \tilde{t}>0, \forall \tau>0, \forall i, \quad \tilde{\rho}_{i}^{(0)}(\tau, \tilde{t})=\tilde{\rho}_{0}^{(0)}(\tilde{t}) .
$$

Proof. By multiplying the equation $(33 \mathrm{a})$ by $\tilde{\rho}_{i}^{(0)}-\tilde{\rho}_{0}^{(0)}$ and by summing over all cells $\Omega_{i}$, we get

$$
\begin{aligned}
& \partial_{\tau}\left(\sum_{i}\left|\tilde{\Omega}_{i}\right|\left(\tilde{\rho}_{i}^{(0)}-\tilde{\rho}_{0}^{(0)}\right)^{2}\right)+\sum_{i} \sum_{\Gamma_{i j} \subset \partial \Omega_{i}}\left|\tilde{\Gamma}_{i j}\right| \tilde{a}_{i j}^{(0)}\left(\tilde{\rho}_{i}^{(0)}-\tilde{\rho}_{0}^{(0)}\right)\left(\tilde{\rho}_{i}^{(0)}-\tilde{\rho}_{j}^{(0)}\right)=0 \\
\Rightarrow & \partial_{\tau}\left(\sum_{i}\left|\tilde{\Omega}_{i}\right|\left(\tilde{\rho}_{i}^{(0)}-\tilde{\rho}_{0}^{(0)}\right)^{2}\right)+\sum_{\Gamma_{i j} \subset \AA}\left|\tilde{\Gamma}_{i j}\right| \tilde{a}_{i j}^{(0)}\left(\tilde{\rho}_{i}^{(0)}-\tilde{\rho}_{j}^{(0)}\right)^{2}+\sum_{\Gamma_{i j} \subset \partial \Omega}\left|\tilde{\Gamma}_{i j}\right| \tilde{a}_{i j}^{(0)}\left(\tilde{\rho}_{i}^{(0)}-\tilde{\rho}_{0}^{(0)}\right)^{2}=0
\end{aligned}
$$


where $\stackrel{\circ}{\Omega}=\Omega \backslash \partial \Omega$ is the interior of $\Omega$. Note that we used that in the boundaries $\partial \Omega, \tilde{\rho}_{j}^{(0)}=\tilde{\rho}_{0}^{(0)}$. Then, we have

$\partial_{\tau}\left(\sum_{i}\left|\tilde{\Omega}_{i}\right|\left(\tilde{\rho}_{i}^{(0)}-\tilde{\rho}_{0}^{(0)}\right)^{2}\right)=-\sum_{\Gamma_{i j} \subset \Omega}\left|\tilde{\Gamma}_{i j}\right| \tilde{a}_{i j}^{(0)}\left(\tilde{\rho}_{i}^{(0)}-\tilde{\rho}_{j}^{(0)}\right)^{2}-\sum_{\Gamma_{i j} \subset \partial \Omega}\left|\tilde{\Gamma}_{i j}\right| \tilde{a}_{i j}^{(0)}\left(\tilde{\rho}_{i}^{(0)}-\tilde{\rho}_{0}^{(0)}\right)^{2} \leq 0$.

Then, the positive function

$$
\tau \mapsto \sum_{i}\left|\tilde{\Omega}_{i}\right|\left(\tilde{\rho}_{i}^{(0)}-\tilde{\rho}_{0}^{(0)}\right)^{2}
$$

decreases. Since at $\tau=0, \tilde{\rho}^{(0)}$ is uniform (equal to $\tilde{\rho}_{0}^{(0)}$ ), this function vanishes at $\tau=0$ and then at any $\tau>0$. This concludes the proof.

Still following the steps of subsection 2.1, the following proposition gives the equivalent of (27) for the semi-discrete scheme

Proposition 2.3 (Consistency with the wave equations). Under the hypothesis of the Proposition 2.2, $\left(\tilde{p}^{(1)} / \gamma,(\tilde{\rho} \tilde{\mathbf{u}})^{(0)}\right)$ satisfies the semi-discrete (discrete in space and continuous in time) wave equation

$$
\left\{\begin{array}{r}
\partial_{\tau} \frac{\tilde{p}_{i}^{(1)}}{\gamma}+\frac{1}{\left|\tilde{\Omega}_{i}\right|} \sum_{\Gamma_{i j} \subset \partial \Omega_{i}}\left|\tilde{\Gamma}_{i j}\right|\left[\left(\tilde{a}^{(0)}\right)^{2} \frac{(\tilde{\rho} \tilde{\mathbf{u}})_{i}^{(0)}+(\tilde{\rho} \tilde{\mathbf{u}})_{j}^{(0)}}{2} \cdot \mathbf{n}_{i j}+\frac{\tilde{a}^{(0)}}{2}\left(\frac{\tilde{p}_{i}^{(1)}}{\gamma}-\frac{\tilde{p}_{j}^{(1)}}{\gamma}\right)\right] \\
=-\frac{1}{\gamma} \mathbf{d}_{\tilde{t}} \tilde{p}^{(0)}, \\
\partial_{\tau}(\tilde{\rho} \tilde{\mathbf{u}})_{i}^{(0)}+\frac{1}{\left|\tilde{\Omega}_{i}\right|} \sum_{\Gamma_{i j} \subset \partial \Omega_{i}}\left|\tilde{\Gamma}_{i j}\right|\left[\frac{\tilde{p}_{i}^{(1)}+\tilde{p}_{j}^{(1)}}{2 \gamma} \mathbf{n}_{i j}\right. \\
\left.+\frac{\delta \tilde{a}^{(0)}}{2}\left[\left((\tilde{\rho} \tilde{\mathbf{u}})_{i}^{(0)}-(\tilde{\rho} \tilde{\mathbf{u}})_{j}^{(0)}\right) \cdot \mathbf{n}_{i j}\right] \mathbf{n}_{i j}\right]=0
\end{array}\right.
$$

where $\delta=1$ for the Roe scheme, $\delta=0$ for the Dellacherie et al. and Rieper fixes and $\tilde{a}^{(0)}=$ $\sqrt{\mathbf{d}_{\tilde{\rho}} \tilde{p}\left(\tilde{\rho}^{(0)}\right) / \gamma}$.

Proof. Under the hypothesis of the Proposition 2.2, we have $\tilde{\rho}_{i}^{(0)}=\tilde{\rho}^{(0)}$ for all $i$. We firstly prove that $\tilde{a}_{i j}^{(0)}=\tilde{a}^{(0)}:=\sqrt{\mathbf{d}_{\tilde{\rho}} \tilde{p}\left(\tilde{\rho}^{(0)}\right) / \gamma}$. If $\tilde{\rho}_{i}=\tilde{\rho}_{j}$, by definition of $a_{i j}$ we have $a_{i j}=a\left(\rho_{i}\right)=\sqrt{\mathbf{d}_{\rho} p\left(\rho_{i}\right)}$ and since $a_{0}^{2}=\gamma p_{0} / \rho_{0}$, we get $\tilde{a}_{i j}=\sqrt{\mathbf{d}_{\tilde{\rho}} \tilde{p}\left(\tilde{\rho}_{i}\right) / \gamma}$ and then $\tilde{a}_{i j}^{(0)}=\sqrt{\mathbf{d}_{\tilde{\rho}} \tilde{p}\left(\tilde{\rho}^{(0)}\right) / \gamma}$. If $\tilde{\rho}_{i} \neq \tilde{\rho}_{j}$, we assume with no loss of generality that $\tilde{\rho}_{i}<\tilde{\rho}_{j}$. By definition of $a_{i j}(10)$ and using the fact that $\tilde{\rho} \mapsto \tilde{p}(\tilde{\rho})$ is a convex function, we have

$$
\begin{array}{r}
\mathbf{d}_{\tilde{\rho}} \tilde{p}\left(\tilde{\rho}_{i}\right) \leq \gamma \tilde{a}_{i j}^{2}=\frac{\tilde{p}\left(\tilde{\rho}_{j}\right)-\tilde{p}\left(\tilde{\rho}_{i}\right)}{\tilde{\rho}_{j}-\tilde{\rho}_{i}} \leq \mathbf{d}_{\tilde{\rho}} \tilde{p}\left(\tilde{\rho}_{j}\right) \\
\Rightarrow \mathbf{d}_{\tilde{\rho}} \tilde{p}\left(\tilde{\rho}_{i}^{(0)}\right)+\mathcal{O}(() M) \leq \gamma\left(\tilde{a}_{i j}^{(0)}\right)^{2}+\mathcal{O}(M) \leq \mathbf{d}_{\tilde{\rho}} \tilde{p}\left(\tilde{\rho}_{j}^{(0)}\right)+\mathcal{O}(M) \\
\Rightarrow \mathbf{d}_{\tilde{\rho}} \tilde{p}\left(\tilde{\rho}_{i}^{(0)}\right) \leq \gamma\left(\tilde{a}_{i j}^{(0)}\right)^{2} \leq \mathbf{d}_{\tilde{\rho}} \tilde{p}\left(\tilde{\rho}_{j}^{(0)}\right)
\end{array}
$$

and since $\tilde{\rho}_{i}^{(0)}=\tilde{\rho}^{(0)}$ for all $i$, we obtain $\tilde{a}_{i j}^{(0)}=\tilde{a}_{i}^{(0)}=\tilde{a}^{(0)}=\sqrt{\mathbf{d}_{\tilde{\rho}} \tilde{p}\left(\tilde{\rho}^{(0)}\right) / \gamma}$ for all $i$.

Then, the equation on $(\tilde{\rho} \tilde{\mathbf{u}})_{i}^{(0)}$ is directly deduced from (33b) and the result of Proposition 2.2. The equation (32a) at order $M^{0}$ and the result of Proposition 2.2 give

$$
\mathbf{d}_{\tilde{t}} \tilde{\rho}^{(0)}+\partial_{\tau} \tilde{\rho}_{i}^{(1)}+\frac{1}{2\left|\tilde{\Omega}_{i}\right|} \sum_{\Gamma_{i j} \subset \partial \Omega_{i}}\left|\tilde{\Gamma}_{i j}\right|\left[\left((\tilde{\rho} \tilde{\mathbf{u}})_{i}^{(0)}+(\tilde{\rho} \tilde{\mathbf{u}})_{j}^{(0)}\right) \cdot \mathbf{n}_{i j}+\tilde{a}^{(0)}\left(\tilde{\rho}_{i}^{(1)}-\tilde{\rho}_{j}^{(1)}\right)\right]=0 .
$$

By multiplying this equation by $\mathbf{d}_{\tilde{\rho}} \tilde{p}\left(\tilde{\rho}^{(0)}\right)=\gamma\left(a^{(0)}\right)^{2}$, we get

$$
\partial_{\tau} \tilde{p}_{i}^{(1)}+\frac{1}{2\left|\tilde{\Omega}_{i}\right|} \sum_{\Gamma_{i j} \subset \partial \Omega_{i}}\left|\tilde{\Gamma}_{i j}\right|\left[\gamma\left(\tilde{a}^{(0)}\right)^{2}\left((\tilde{\rho} \tilde{\mathbf{u}})_{i}^{(0)}+(\tilde{\rho} \tilde{\mathbf{u}})_{j}^{(0)}\right) \cdot \mathbf{n}_{i j}+\tilde{a}^{(0)}\left(\tilde{p}_{i}^{(1)}-\tilde{p}_{j}^{(1)}\right)\right]=-\mathbf{d}_{\bar{t}} \tilde{p}^{(0)}
$$

because

$$
\mathbf{d}_{\tilde{\rho}} \tilde{p}\left(\tilde{\rho}^{(0)}\right) \mathbf{d}_{\tilde{t}} \tilde{\rho}^{(0)}=\mathbf{d}_{\hat{t}} \tilde{p}^{(0)}, \quad \tilde{\rho}^{(0)}(\tau, \tilde{t})=\tilde{\rho}^{(0)}(\tilde{t}) \quad \text { and } \quad \forall i, \mathbf{d}_{\tilde{\rho}} \tilde{p}\left(\tilde{\rho}^{(0)}\right) \tilde{\rho}_{i}^{(1)}=\tilde{p}_{i}^{(1)} .
$$

This concludes the proof. 
Once the consistency with the wave equations has been proved, we are now interested in explaining the results observed in Figure 3. For simplifying, we denote by $\mathbf{m}=(\rho \tilde{\mathbf{u}})^{(0)}, p=\tilde{p}^{(1)} / \gamma$ and $a_{\star}=\tilde{a}^{(0)}$ in the semi-discrete scheme (34). The domain is supposed to be infinite or periodic, so that $\tilde{p}^{(0)}(t)$ is equal to 0 for any $t$ (following Proposition 2.2). Then (34), discretized in space with a uniform mesh with step $\Delta x$, and in time with the forward Euler scheme is

$$
\left\{\begin{array}{l}
\frac{p_{i}^{n+1}-p_{i}^{n}}{\Delta \tau}+a_{\star}^{2} \frac{m_{i+1}^{n}-m_{i-1}^{n}}{2 \Delta x}-\frac{a_{\star} \Delta x}{2} \frac{p_{i+1}^{n}-2 p_{i}^{n}+p_{i-1}^{n}}{(\Delta x)^{2}}=0 \\
\frac{m_{i}^{n+1}-m_{i}^{n}}{\Delta \tau}+\frac{p_{i+1}^{n}-p_{i-1}^{n}}{2 \Delta x}-\frac{\delta a_{\star} \Delta x}{2} \frac{m_{i+1}^{n}-2 m_{i}^{n}+m_{i-1}^{n}}{(\Delta x)^{2}}=0
\end{array}\right.
$$

where $\delta=1$ for the Roe scheme and $\delta=0$ for the Dellacherie et al. or Rieper fixes.

Proposition 2.4 (CFL condition). The explicit one dimensional Roe scheme ((35) with $\delta=1)$ is $L^{2}$ stable under the CFL condition

$$
0 \leq a_{\star} \frac{\Delta \tau}{\Delta x} \leq 1
$$

The explicit one dimensional Roe scheme corrected with Dellacherie et al. or Rieper fixes ((35) with $\delta=0$ ) is $L^{2}$ stable under the degenerate $C F L$ condition

$$
0 \leq 2 a_{\star} \frac{\Delta \tau}{\Delta x} \leq 1
$$

Proof. This result is just an application of [11, Lemma 5.1 p. 994] obtained with a von Neumann analysis. [11, Lemma 5.1 p. 994] depends on the variables $r, u, M, \Delta \tau, \kappa_{r}, \kappa_{u}$. We set $r=p$, $u=a_{\star} m, M=1, \Delta \tau=\Delta t$. Setting $\kappa_{r}=\kappa_{u}=1$ leads to (36) and setting $\kappa_{r}=1$ and $\kappa_{u}=0$ gives $(37)$.

Last, concerning the wrong order of accuracy observed in subsubsection 2.2.2, we do not have a full explanation, but can do the following remark

Remark 2.5. In [7], the $p+1$ order of accuracy for a polynomial degree of $p$ is obtained by proving a $p$ order on the gradients, which is obtained by an inverse inequality. This comes from the fact that the numerical diffusion operator is strictly non-negative. In the stabilization of the wave equation with Dellacherie et al. or Rieper fixes, the diffusion tensor is nonnegative, but not strictly, and involves only the density, but not the momentum. This formally explains why we have the right order on the density but not on the momentum.

In this section, we proved that the usual low Mach number fixes are not well suited with the computation of acoustic waves: the CFL number is lower than the expected one, and the order of accuracy with a discontinuous Galerkin scheme does not give the right order of accuracy for momentum. On the other hand, the original Roe scheme gives good results on the one dimensional low Mach acoustic test, but is known for being inaccurate for steady low Mach number flow calculations. Next section is dedicated to the development of a low Mach number fix which is accurate for steady computations, and which gives the right order of accuracy for acoustic unsteady computations with the second order discontinuous Galerkin method.

\section{Construction of a new scheme: accurate in the low Mach number limit for steady and for acoustics computations}

The aim of this section is to develop a low Mach number fix which is accurate for steady computations, and which gives the right order of accuracy for acoustics computations with the second order discontinuous Galerkin method. We first focus on (28), which, with the change of variables $\mathbf{m}=(\rho \tilde{\mathbf{u}})^{(0)}, p=\tilde{p}^{(1)} / \gamma$ and $a_{\star}=\tilde{a}^{(0)}$, can be expressed as

$$
\left\{\begin{array}{l}
\partial_{\tau} p+a_{\star}^{2} \nabla \cdot \mathbf{m}=0, \\
\partial_{t} \mathbf{m}+\nabla p=0 .
\end{array}\right.
$$

and for which the semi-discrete asymptotic expansion gives the following discretisation

$$
\left\{\begin{array}{l}
\frac{p_{i}^{n+1}-p_{i}^{n}}{\Delta \tau}+\frac{1}{\left|\tilde{\Omega}_{i}\right|} \sum_{\Gamma_{i j} \subset \partial \Omega_{i}}\left|\tilde{\Gamma}_{i j}\right|\left[a_{\star}^{2} \frac{\mathbf{m}_{i}^{n}+\mathbf{m}_{j}^{n}}{2} \cdot \mathbf{n}_{i j}+\frac{a_{\star}}{2}\left(p_{i}^{n}-p_{j}^{n}\right)\right]=0, \\
\frac{\mathbf{m}_{i}^{n+1}-\mathbf{m}_{i}^{n}}{\Delta \tau}+\frac{1}{\left|\tilde{\Omega}_{i}\right|} \sum_{\Gamma_{i j} \subset \partial \Omega_{i}}\left|\tilde{\Gamma}_{i j}\right|\left[\frac{p_{i}^{n}+p_{j}^{n}}{2} \mathbf{n}_{i j}+\frac{\delta a_{\star}}{2}\left[\left(\mathbf{m}_{i}^{n}-\mathbf{m}_{j}^{n}\right) \cdot \mathbf{n}_{i j}\right] \mathbf{n}_{i j}\right]=0 .
\end{array}\right.
$$


with $\delta=1$ for the Roe scheme, and $\delta=0$ for the fix of Dellacherie et al. and the fix of Rieper. In subsection 3.1, a new set of dissipative terms is chosen for (39) in order to avoid velocity diffusion in the pressure equation, and to ensure the same CFL stability criterion as the Roe scheme. Then, in subsection 3.2, the scheme is extended to the barotropic Euler equations.

\subsection{Development of a scheme for the wave system (38)}

In this section, the general expression of the scheme is given in subsubsection 3.1.1. This expression depends on some coefficients, which are progressively fixed: in subsubsection 3.1.2, necessary conditions on the coefficients are found for ensuring the energy stability, then in subsubsection 3.1.3, a condition for (formally) ensuring accuracy for steady states is given. In subsubsection 3.1.4, coefficients for ensuring optimal CFL are found in one dimension. This is then extended in two dimensions in subsubsection 3.1.5. Last, in subsubsection 3.1.6, the final expression of the scheme for the wave system is given.

\subsubsection{General expression of the new scheme}

The Roe scheme is not accurate at low Mach number. The different fixes proposed so far for steady computations have drawbacks for the computation of acoustics: the Roe-Turkel scheme dissipates very quickly the density, whereas the Rieper or Dellacherie et al. fixes allow to do acoustic computations, but the stability CFL number is low, and the optimal order is not reached for a second order discontinuous Galerkin scheme.

We now want to choose the diffusion of the scheme to make it accurate at low Mach number, stable under the same CFL restriction as the Roe scheme. The new scheme can be written as

$$
\left\{\begin{aligned}
& \frac{p_{i}^{n+1}-p_{i}^{n}}{\Delta \tau}+\frac{1}{\left|\tilde{\Omega}_{i}\right|} \sum_{\Gamma_{i j} \subset \partial \Omega_{i}}\left|\tilde{\Gamma}_{i j}\right|[ a_{\star}^{2} \frac{\mathbf{m}_{i}^{n}+\mathbf{m}_{j}^{n}}{2} \cdot \mathbf{n}_{i j}+C_{11}\left(p_{i}^{n}-p_{j}^{n}\right) \\
&+\mathbf{C}_{12, d} \cdot \mathbf{n}_{i j}\left(\mathbf{m}_{i}^{n}-\mathbf{m}_{j}^{n}\right) \cdot \mathbf{n}_{i j}+\mathbf{C}_{12, d} \cdot \mathbf{t}_{i j}^{a}\left(\mathbf{m}_{i}^{n}-\mathbf{m}_{j}^{n}\right) \cdot \mathbf{t}_{i j}^{a} \\
&\left.+\mathbf{C}_{12, d} \cdot \mathbf{t}_{i j}^{b}\left(\mathbf{m}_{i}^{n}-\mathbf{m}_{j}^{n}\right) \cdot \mathbf{t}_{i j}^{b}\right]=0, \\
& \frac{\mathbf{m}_{i}^{n+1}-\mathbf{m}_{i}^{n}}{\Delta \tau}+\frac{1}{\left|\tilde{\Omega}_{i}\right|} \sum_{\Gamma_{i j} \subset \partial \Omega_{i}}\left|\tilde{\Gamma}_{i j}\right|\left[\frac{p_{i}^{n}+p_{j}^{n}}{2} \mathbf{n}_{i j}+\mathbf{C}_{21, d} \cdot \mathbf{n}_{i j}\left(p_{i}^{n}-p_{j}^{n}\right) \mathbf{n}_{i j}\right. \\
&+\mathbf{C}_{21, d} \cdot \mathbf{t}_{i j}^{a}\left(p_{i}^{n}-p_{j}^{n}\right) \mathbf{t}_{i j}^{a}+\mathbf{C}_{21, d} \cdot \mathbf{t}_{i j}^{b}\left(p_{i}^{n}-p_{j}^{n}\right) \mathbf{t}_{i j}^{b} \\
&\left.+C_{22} \mathbf{n}_{i j} \otimes \mathbf{n}_{i j}\left(\mathbf{m}_{i}^{n}-\mathbf{m}_{j}^{n}\right)\right]=0
\end{aligned}\right.
$$

where $C_{11}$ and $C_{22}$ are scalar, $\mathbf{C}_{12, d}$ and $\mathbf{C}_{21, d}$ are vectors of the size of the space dimension $d$ and $\left(\mathbf{n}, \mathbf{t}^{a}, \mathbf{t}^{b}\right)$ defines an orthonormal basis of $\mathbb{R}^{3}$. This general expression was first proposed in [1] but here we also add tangential diffusion. We want choose $C_{11}, \mathbf{C}_{21, d}, \mathbf{C}_{12, d}$ and $C_{22}$ such that the scheme is accurate at low Mach number for steady problems, and stable for acoustics computations in a low Mach number flow with the same CFL condition as the Roe scheme. The Roe scheme matches with $C_{11}=C_{22}=a_{\star} / 2$ and $\mathbf{C}_{12, d}=\mathbf{C}_{21, d}=0$. The Roe scheme corrected with Dellacherie et al. or Rieper fixes matches with $C_{11}=a_{\star} / 2, \mathbf{C}_{12, d}=\mathbf{C}_{21, d}=0$ and $C_{22}=0$. Note that the system (40) can also be written as

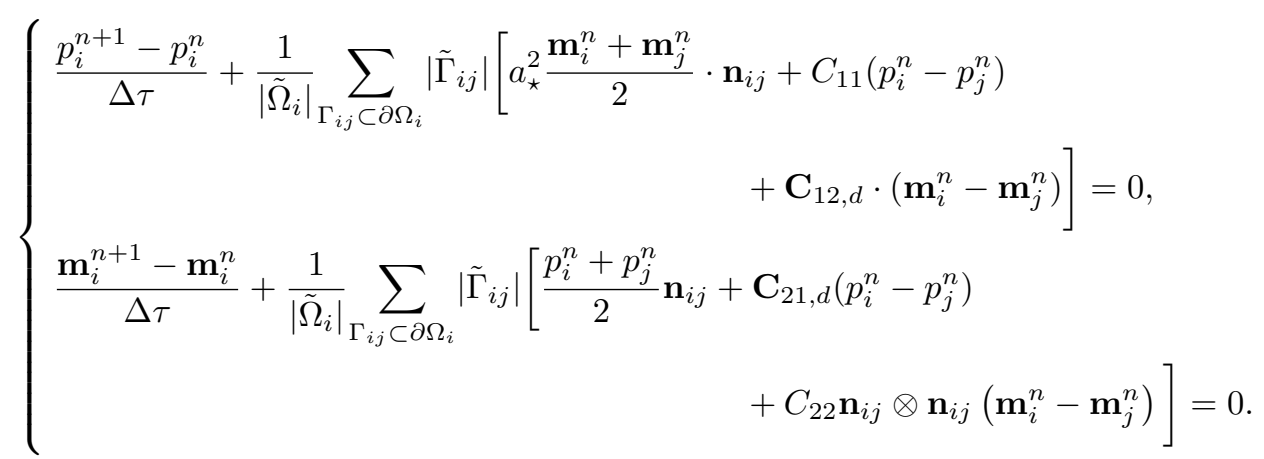

The coefficients of (41) will be progressively fixed, based on stability requirements. Next section is dedicated to conditions for ensuring the energy inequality. 


\subsubsection{Conditions for ensuring a semi-discrete energy inequality}

We want a condition on $C_{11}, \mathbf{C}_{12, d}, \mathbf{C}_{21, d}$ and $C_{22}$ such that the new scheme (41) satisfies a semidiscrete (continuous in time and discrete in space) energy inequality on a periodic domain. In the one dimensional case, $\mathbf{C}_{12, d}$ and $\mathbf{C}_{21, d}$ are scalars that we note $C_{12, d=1}$ and $C_{21, d=1}$.

Proposition 3.1. The scheme (41) satisfies the semi-discrete energy inequality

$$
\partial_{\tau}\left(\sum_{i}\left|\tilde{\Omega}_{i}\right| \frac{p_{i}^{2}+a_{\star}^{2}\left\|\mathbf{m}_{i}\right\|^{2}}{2}\right) \leq 0
$$

for any sequence $(p, \mathbf{m})_{i}$ and any mesh on a periodic domain if and only if

$$
\left\{\begin{array}{l}
C_{11} \geq 0 \\
C_{22} \geq 0 \\
\left(C_{12, d=1}+a_{\star}^{2} C_{21, d=1}\right)^{2} \leq 4 C_{11} C_{22}
\end{array}\right.
$$

for $d=1$ and if and only if

$$
\left\{\begin{array}{l}
C_{11} \geq 0 \\
\mathbf{C}_{12, d}+a_{\star}^{2} \mathbf{C}_{21, d}=0 \\
C_{22} \geq 0
\end{array}\right.
$$

for $d>1$.

Proof. Since

$$
\sum_{\Gamma_{i j} \subset \partial \Omega_{i}}\left|\tilde{\Gamma}_{i j}\right| \mathbf{n}_{i j}=0
$$

the semi-discrete version of the equation of (41a) can be written as

$$
\partial_{\tau} p_{i}+\frac{1}{\left|\tilde{\Omega}_{i}\right|} \sum_{\Gamma_{i j} \subset \partial \Omega_{i}}\left|\tilde{\Gamma}_{i j}\right|\left[\frac{a_{\star}^{2}}{2} \mathbf{m}_{j} \cdot \mathbf{n}_{i j}+C_{11}\left(p_{i}-p_{j}\right)+\mathbf{C}_{12, d} \cdot\left(\mathbf{m}_{i}-\mathbf{m}_{j}\right)\right]=0 .
$$

By multiplying this equation by $\left|\tilde{\Omega}_{i}\right| p_{i}$ and by summing over all cells, since all edges are counted twice (one time for the cell $\Omega_{i}$ and one time for the cell $\Omega_{j}$ ), we obtain

$$
\begin{aligned}
\partial_{\tau}\left(\sum_{i}\left|\tilde{\Omega}_{i}\right| \frac{p_{i}^{2}}{2}\right)+\sum_{\Gamma_{i j}}\left|\tilde{\Gamma}_{i j}\right|\left[\frac{a_{\star}^{2}}{2} p_{i} \mathbf{m}_{j}^{n} \cdot \mathbf{n}_{i j}\right. & +\frac{a_{\star}^{2}}{2} p_{j} \mathbf{m}_{i} \cdot \mathbf{n}_{j i}+C_{11} p_{i}\left(p_{i}-p_{j}\right)+C_{11} p_{j}\left(p_{j}-p_{i}\right) \\
& \left.+p_{i} \mathbf{C}_{12, d} \cdot\left(\mathbf{m}_{i}-\mathbf{m}_{j}\right)+p_{j} \mathbf{C}_{12, d} \cdot\left(\mathbf{m}_{j}-\mathbf{m}_{i}\right)\right]=0 .
\end{aligned}
$$

Since $\mathbf{n}_{j i}=-\mathbf{n}_{i j}$, it gives

$$
\begin{aligned}
\partial_{\tau}\left(\sum_{i}\left|\tilde{\Omega}_{i}\right| \frac{p_{i}^{2}}{2}\right)+\sum_{\Gamma_{i j}}\left|\tilde{\Gamma}_{i j}\right|\left[\frac{a_{\star}^{2}}{2} p_{i} \mathbf{m}_{j}^{n} \cdot \mathbf{n}_{i j}-\frac{a_{\star}^{2}}{2} p_{j} \mathbf{m}_{i} \cdot \mathbf{n}_{i j}\right. & +C_{11}\left(p_{i}-p_{j}\right)^{2} \\
& \left.+\left(p_{i}-p_{j}\right) \mathbf{C}_{12, d} \cdot\left(\mathbf{m}_{i}-\mathbf{m}_{j}\right)\right]=0 .
\end{aligned}
$$

Since (45) is satisfied, the semi-discrete version of the equation of (41b) is given by

$$
\partial_{\tau} \mathbf{m}_{i}+\frac{1}{\left|\tilde{\Omega}_{i}\right|} \sum_{\Gamma_{i j} \subset \partial \Omega_{i}}\left|\tilde{\Gamma}_{i j}\right|\left[\frac{p_{j}}{2} \mathbf{n}_{i j}+\mathbf{C}_{21, d}\left(p_{i}-p_{j}\right)+C_{22}\left(\left(\mathbf{m}_{i}-\mathbf{m}_{j}\right) \cdot \mathbf{n}_{i j}\right) \cdot \mathbf{n}_{i j}\right]=0 .
$$

By multiplying this equation by $\left|\tilde{\Omega}_{i}\right| \mathbf{m}_{i}$ and by summing over all cells, we get

$$
\begin{aligned}
& \partial_{\tau}\left(\sum_{i}\left|\tilde{\Omega}_{i}\right| \frac{\left\|\mathbf{m}_{i}\right\|^{2}}{2}\right)+\sum_{\Gamma_{i j}}\left|\tilde{\Gamma}_{i j}\right|\left[\frac{1}{2} p_{j} \mathbf{m}_{i} \cdot \mathbf{n}_{i j}+\frac{1}{2} p_{i} \mathbf{m}_{j} \cdot \mathbf{n}_{j i}+\left(p_{i}-p_{j}\right) \mathbf{C}_{21, d} \cdot \mathbf{m}_{i}\right. \\
& \left.\quad+\left(p_{j}-p_{i}\right) \mathbf{C}_{21, d} \cdot \mathbf{m}_{j}+C_{22}\left(\left(\mathbf{m}_{i}-\mathbf{m}_{j}\right) \cdot \mathbf{n}_{i j}\right) \mathbf{m}_{i} \cdot \mathbf{n}_{i j}+C_{22}\left(\left(\mathbf{m}_{j}-\mathbf{m}_{i}\right) \cdot \mathbf{n}_{j i}\right) \mathbf{m}_{j} \cdot \mathbf{n}_{j i}\right]=0 .
\end{aligned}
$$

Since $\mathbf{n}_{j i}=-\mathbf{n}_{i j}$, it gives

$$
\begin{aligned}
\partial_{\tau}\left(\sum_{i}\left|\tilde{\Omega}_{i}\right| \frac{\left\|\mathbf{m}_{i}\right\|^{2}}{2}\right)+\sum_{\Gamma_{i j}}\left|\tilde{\Gamma}_{i j}\right|\left[\frac{1}{2} p_{j} \mathbf{m}_{i} \cdot \mathbf{n}_{i j}-\frac{1}{2} p_{i} \mathbf{m}_{j} \cdot \mathbf{n}_{i j}+\right. & \left(p_{i}-p_{j}\right) \mathbf{C}_{21, d} \cdot\left(\mathbf{m}_{i}-\mathbf{m}_{j}\right) \\
& \left.+C_{22}\left(\left(\mathbf{m}_{i}-\mathbf{m}_{j}\right) \cdot \mathbf{n}_{i j}\right)^{2}\right]=0 .
\end{aligned}
$$


We multiply this equation by $a_{\star}^{2}$ and we sum it with equation (46). We obtain

$$
\begin{aligned}
\partial_{\tau}\left(\sum_{i}\left|\tilde{\Omega}_{i}\right| \frac{p_{i}^{2}+a_{\star}^{2}\left\|\mathbf{m}_{i}\right\|^{2}}{2}\right)+\sum_{\Gamma_{i j}}\left|\tilde{\Gamma}_{i j}\right|\left[C_{11}\left(p_{i}-p_{j}\right)^{2}\right. \\
\left.+\left(p_{i}-p_{j}\right)\left(\mathbf{C}_{12, d}+a_{\star}^{2} \mathbf{C}_{21, d}\right) \cdot\left(\mathbf{m}_{i}-\mathbf{m}_{j}\right)+C_{22}\left(\left(\mathbf{m}_{i}-\mathbf{m}_{j}\right) \cdot \mathbf{n}_{i j}\right)^{2}\right]=0
\end{aligned}
$$

which ends the proof.

In this section, first conditions on the coefficients have been found for ensuring energy inequality. Next section is dedicated to refine these conditions for ensuring accuracy at low Mach number in the steady case.

\subsubsection{Accuracy at low Mach number in the steady case}

All the previous schemes which are accurate at low Mach number in the steady case match with $C_{22}=0$. Then, we will also choose

$$
C_{22}=0 .
$$

This choice could be justified by studying the consistency error on Cartesian meshes as it is done in [8]. Here, we do not prove that this choice induces that the resulting scheme will be accurate at low Mach number. However, we will test the accuracy property at low Mach number in the steady case from a numerical point of view in the subsubsection 4.2.3. Next section is dedicated to find exactly the coefficients in one dimension, based on the conditions found in subsubsection 3.1.2 and subsubsection 3.1.3.

\subsubsection{Stability analysis of the explicit new scheme in dimension one}

The previous studies give, if $d>1$,

$$
C_{11} \geq 0, \quad \mathbf{C}_{12, d}=-a_{\star}^{2} \mathbf{C}_{21, d} \quad \text { and } \quad C_{22}=0 .
$$

Since we want the same scheme in dimension one and in dimension two or three, we also assume that (48) holds in dimension one. It remains to choose $C_{11}$ and $C_{12, d=1}$ to get a stable scheme, and a von Neumann analysis of the explicit scheme in dimension one is performed for finding these coefficients. In dimension one, the new scheme can be written in the following form

$$
\left\{\begin{array}{l}
\frac{p_{i}^{n+1}-p_{i}^{n}}{\Delta \tau}+a_{\star}^{2} \frac{m_{i+1}^{n}-m_{i-1}^{n}}{2 \Delta x}-C_{11} \frac{p_{i+1}^{n}-2 p_{i}^{n}+p_{i-1}^{n}}{\Delta x} \\
\frac{m_{i}^{n+1}-m_{i}^{n}}{\Delta \tau}+\frac{p_{i+1}^{n}-p_{i-1}^{n}}{2 \Delta x}-C_{21, d=1}^{n} \frac{p_{i+1}^{n}-2 p_{i}^{n}+p_{i-1}^{n}}{\Delta x}=0
\end{array}\right.
$$

with $C_{12, d=1}=-a_{\star}^{2} C_{21, d=1}$. We aim at finding $C_{11}$ and $C_{21, d=1}$ such that the CFL stability number is the same as the explicit Roe scheme (see Proposition 2.4)

$$
0 \leq a_{\star} \frac{\Delta \tau}{\Delta x} \leq 1 .
$$

The optimal $C_{11}$ and $C_{21, d=1}$ coefficients are given in the following proposition.

Proposition 3.2. The scheme (49) with $C_{12, d=1}=-a_{\star}^{2} C_{21, d=1}$ is stable under the CFL condition (50) if and only if

$$
\left\{\begin{array}{l}
C_{11}=a_{\star}, \\
C_{21, d=1}= \pm \frac{1}{2} .
\end{array}\right.
$$

Proof. By substituting the discrete Fourier modes

$$
p_{i}^{n}=\varphi_{p}^{n} e^{\mathrm{j}\left(k x_{i}\right)}, \quad m_{i}^{n}=\varphi_{m}^{n} e^{\mathrm{j}\left(k x_{i}\right)}
$$

into the fully discrete scheme, we obtain

$$
\varphi^{n+1}=A_{1} \varphi^{n}
$$


where $\varphi=\left(\varphi_{p}, \varphi_{m}\right)^{T}$ and the matrix $A_{1}$ is given by

$A_{1}=\left(\begin{array}{cc}1-4 C_{11} \frac{\Delta \tau}{\Delta x} \sin ^{2}\left(\frac{k \Delta x}{2}\right) & -4 C_{12, d=1} \frac{\Delta \tau}{\Delta x} \sin ^{2}\left(\frac{k \Delta x}{2}\right)-\mathrm{j} a_{\star}^{2} \frac{\Delta \tau}{\Delta x} \sin (k \Delta x) \\ -4 C_{21, d=1} \frac{\Delta \tau}{\Delta x} \sin ^{2}\left(\frac{k \Delta x}{2}\right)-\mathrm{j} \frac{\Delta \tau}{\Delta x} \sin (k \Delta x) & 1\end{array}\right)$.

We want a condition on $C_{11}, C_{12, d=1}$ and $C_{21, d=1}$ such that the eigenvalues of the matrix $A_{1}$ are in the unit disc. We have

$$
\begin{aligned}
\operatorname{det}\left(A_{1}-\lambda \mathrm{I}_{2}\right) & =\lambda^{2}-\operatorname{Tr}\left(A_{1}\right) \lambda+\operatorname{det}\left(A_{1}\right) \\
& =\lambda^{2}+\gamma_{1} \lambda+\beta_{1}
\end{aligned}
$$

with

$$
\begin{aligned}
\gamma_{1}= & -\operatorname{Tr}\left(A_{1}\right)=-\left(2-4 C_{11} \frac{\Delta \tau}{\Delta x} \sin ^{2}\left(\frac{k \Delta x}{2}\right)\right) \\
\beta_{1}= & \operatorname{det}\left(A_{1}\right)=1-4 C_{11} \frac{\Delta \tau}{\Delta x} \sin ^{2}\left(\frac{k \Delta x}{2}\right)+16 a_{\star}^{2} C_{21, d=1}^{2}\left(\frac{\Delta \tau}{\Delta x}\right)^{2} \sin ^{4}\left(\frac{k \Delta x}{2}\right) \\
& +a_{\star}^{2}\left(\frac{\Delta \tau}{\Delta x}\right)^{2} \sin ^{2}(k \Delta x) .
\end{aligned}
$$

We note $\lambda_{ \pm}$the two roots. In order to ensure that the two roots are in the unit disc $\left|\lambda_{ \pm}\right| \leq 1$, the coefficients $\gamma_{1}$ and $\beta_{1}$ have to satisfy

$$
\left\{\begin{array}{l}
\beta_{1} \leq 1 \\
\left|\gamma_{1}\right| \leq 1+\beta_{1}
\end{array}\right.
$$

We have

$$
\begin{aligned}
& \beta_{1} \leq 1 \\
\Leftrightarrow & 4 C_{11} \frac{\Delta \tau}{\Delta x} \sin ^{2}\left(\frac{k \Delta x}{2}\right)-16 a_{\star}^{2} C_{21, d=1}^{2}\left(\frac{\Delta \tau}{\Delta x}\right)^{2} \sin ^{4}\left(\frac{k \Delta x}{2}\right)-a_{\star}^{2}\left(\frac{\Delta \tau}{\Delta x}\right)^{2} \sin ^{2}(k \Delta x) \geq 0 \\
\Leftrightarrow & \frac{\Delta \tau}{\Delta x} \sin ^{2}\left(\frac{k \Delta x}{2}\right)\left[C_{11}-a_{\star}\left(a_{\star} \frac{\Delta \tau}{\Delta x}\right)+a_{\star}^{2} \frac{\Delta \tau}{\Delta x} \sin ^{2}\left(\frac{k \Delta x}{2}\right)\left(1-4 C_{21, d=1}^{2}\right)\right] \geq 0
\end{aligned}
$$

where the equality $\sin ^{2}(a)=(2 \sin (a / 2) \cos (a / 2))^{2}=4 \sin ^{2}(a / 2)-4 \sin ^{4}(a / 2)$ was used between the second and third line. Since the inequality obtained should be satisfied for all $0 \leq a_{\star} \Delta \tau / \Delta x \leq 1$ and for all $k \Delta x \in \mathbb{R}$, we get

$$
\beta_{1} \leq 1 \Leftrightarrow\left\{\begin{array} { l } 
{ C _ { 1 1 } - a _ { \star } \geq 0 } \\
{ 1 - 4 C _ { 2 1 , d = 1 } ^ { 2 } \geq 0 }
\end{array} \Leftrightarrow \left\{\begin{array}{l}
C_{11} \geq a_{\star} \\
\left|C_{21, d=1}\right| \leq \frac{1}{2}
\end{array}\right.\right.
$$

We also have

$$
\begin{aligned}
& \left|\gamma_{1}\right| \leq 1+\beta_{1} \Rightarrow-\gamma_{1} \leq 1+\beta_{1} \\
\Rightarrow & 4-8 C_{11} \frac{\Delta \tau}{\Delta x} \sin ^{2}\left(\frac{k \Delta x}{2}\right)+16 a_{\star}^{2} C_{21, d=1}^{2}\left(\frac{\Delta \tau}{\Delta x}\right)^{2} \sin ^{4}\left(\frac{k \Delta x}{2}\right)+a_{\star}^{2}\left(\frac{\Delta \tau}{\Delta x}\right)^{2} \sin ^{2}(k \Delta x) \geq 0 \\
\Rightarrow & 1 \geq\left(a_{\star} \frac{\Delta \tau}{\Delta x}\right) \sin ^{2}\left(\frac{k \Delta x}{2}\right)\left[2 \frac{C_{11}}{a_{\star}}-a_{\star} \frac{\Delta \tau}{\Delta x}+a_{\star} \frac{\Delta \tau}{\Delta x} \sin ^{2}\left(\frac{k \Delta x}{2}\right)\left(1-4 C_{21, d=1}^{2}\right)\right]
\end{aligned}
$$

where the equality $\sin ^{2}(a)=(2 \sin (a / 2) \cos (a / 2))^{2}=4 \sin ^{2}(a / 2)-4 \sin ^{4}(a / 2)$ was used between the second and third line. The obtained inequality should be satisfied for all for all $0 \leq a_{\star} \Delta \tau / \Delta x \leq 1$ and for all $k \Delta x \in \mathbb{R}$, and we choose the parameters $k, \Delta \tau$ and $\Delta x$ such that

$$
a_{\star} \Delta \tau / \Delta x=1 \quad \text { and } \quad \sin ^{2}\left(\frac{k \Delta x}{2}\right)=1,
$$

which gives the inequality

$$
1 \geq 2 \frac{C_{11}}{a_{\star}}-4 C_{21, d=1}^{2}
$$


By combining (54) and (55), we get

$$
\left\{\begin{array}{l}
C_{11} \leq a_{\star} \\
\left|C_{21, d=1}\right| \geq \frac{1}{2}
\end{array}\right.
$$

Then, with (54), we obtain that if the scheme is stable under the CFL condition (50), then $C_{11}$ and $C_{21, d=1}$ satisfy (51).

We now check that if $C_{11}=a_{\star}, C_{21, d=1}= \pm 1 / 2$ and $C_{12, d=1}=-a_{\star}^{2} C_{21, d=1}$, the eigenvalues $\lambda_{ \pm}$ are in the unit disc under the CFL condition (50). We easily obtain that

$$
\lambda_{ \pm}=1-2 a_{\star} \frac{\Delta \tau}{\Delta x} \sin ^{2}\left(\frac{k \Delta x}{2}\right) \pm \mathrm{j} a_{\star} \frac{\Delta \tau}{\Delta x} \sin (k \Delta x) .
$$

Then

$$
\left|\lambda_{ \pm}\right|^{2}=1-4 a_{\star} \frac{\Delta \tau}{\Delta x} \sin ^{2}\left(\frac{k \Delta x}{2}\right)\left(1-a_{\star} \frac{\Delta \tau}{\Delta x}\right)
$$

and we get $\left|\lambda_{ \pm}\right| \leq 1$ if and only if the CFL condition (50) is satisfied.

Remark 3.3. The sign choice in (51) gives actually a choice between two schemes that optimize the CFL number. Each of these schemes are not Galilean invariant. In one dimension, the only rotation that exists is the multiplication of all the vectors by -1 . Then applying this rotation to one scheme gives the other one, and vice versa.

Remark 3.4 (Comparison of the dissipation of the semi-discrete scheme with other discretizations). We denote by

$$
S=\left(\begin{array}{cc}
1 & 0 \\
0 & a_{\star}^{2}
\end{array}\right)
$$

the symmetrizer of the acoustic wave system, and by $\varphi_{k}$ the $k^{\text {th }}$ Fourier mode of the semi-discrete scheme

$$
\mathbf{d}_{\tau} \varphi_{k}=A(k) \varphi_{k} .
$$

Then the energy of the $k^{\text {th }}$ Fourier mode follows

$$
\mathbf{d}_{\tau}\left({\overline{\varphi_{k}}}^{T} S \varphi_{k}\right)={\overline{\varphi_{k}}}^{T}\left(\overline{A(k)}^{T} S+S A(k)\right) \varphi_{k} .
$$

For the Roe scheme, we find

$$
\mathbf{d}_{\tau}\left(\bar{\varphi}_{k}^{T} S \varphi_{k}\right)=-\frac{2 a_{\star} \sin ^{2}\left(\frac{k \Delta x}{2}\right)}{\Delta x} \bar{\varphi}_{k}^{T} S \varphi_{k} .
$$

whereas with the fixes of [12, 47], we find

$$
\mathbf{d}_{\tau}\left(\bar{\varphi}_{k}^{T} S \varphi_{k}\right)=-\frac{2 a_{\star} \sin ^{2}\left(\frac{k \Delta x}{2}\right)}{\Delta x} \bar{\varphi}_{k}^{T}\left(\begin{array}{ll}
1 & 0 \\
0 & 0
\end{array}\right) \varphi_{k}
$$

and with the new fix

$$
\mathbf{d}_{\tau}\left({\overline{\varphi_{k}}}^{T} S \varphi_{k}\right)=-\frac{4 a_{\star} \sin ^{2}\left(\frac{k \Delta x}{2}\right)}{\Delta x} \bar{\varphi}_{k}^{T}\left(\begin{array}{cc}
1 & 0 \\
0 & 0
\end{array}\right) \varphi_{k} .
$$

Then using that $\left(\begin{array}{ll}1 & 0 \\ 0 & 0\end{array}\right) \leq S$ in the Lowner order, we conclude that with the fix of [12, 47], the energy dissipation is lower than with the Roe scheme, whereas with the new fix, the energy dissipation is lower than twice the one of the Roe scheme. Note, however, that the dissipation of the fix of [12, 47] does not allow to get the optimal order with a second order scheme, and has a CFL number twice lower than the new scheme and the original Roe scheme.

Until now, we have fully determined the scheme in one dimension. Next section is dedicated to extend the scheme in two (and three) dimension. 


\subsubsection{Stability analysis of the explicit new scheme in dimension two}

The aim of this section is to extend the scheme in two dimension. The study is limited to two dimensional regular Cartesian meshes. As the scheme is not Galilean invariant, the extension to two dimensions is not straightforward.

3.1.5.1 Modified equation on a Cartesian mesh in dimension two We write the modified equation in dimension two and not three because of the readability. We assume that the mesh is a regular Cartesian mesh with $\Delta x=\Delta y$. The unknowns are $(p, \mathbf{m})_{i, j}$ where $\mathbf{m}=\left(m_{x}, m_{y}\right)^{T}, i$ is the line index and $j$ the column index. The equation on $p$ of the scheme (41) can be written as

$$
\begin{gathered}
\frac{p_{i, j}^{n+1}-p_{i, j}^{n}}{\Delta \tau}+a_{\star}^{2} \frac{\left(m_{x}\right)_{i+1, j}-\left(m_{x}\right)_{i-1, j}}{2 \Delta x}+a_{\star}^{2} \frac{\left(m_{y}\right)_{i, j+1}-\left(m_{y}\right)_{i, j-1}}{2 \Delta x} \\
-C_{11} \frac{p_{i+1, j}^{n}-2 p_{i, j}^{n}+p_{i-1, j}^{n}}{\Delta x}-C_{11} \frac{p_{i, j+1}^{n}-2 p_{i, j}^{n}+p_{i, j-1}^{n}}{\Delta x} \\
+\frac{1}{\Delta x} \mathbf{C}_{12, d=2} \cdot\left(\begin{array}{c}
-\left(m_{x}\right)_{i+1, j}+2\left(m_{x}\right)_{i, j}-\left(m_{x}\right)_{i-1, j}-\left(m_{x}\right)_{i, j+1}+2\left(m_{x}\right)_{i, j}-\left(m_{x}\right)_{i, j-1} \\
-\left(m_{y}\right)_{i+1, j}+2\left(m_{y}\right)_{i, j}-\left(m_{y}\right)_{i-1, j}-\left(m_{y}\right)_{i, j+1}+2\left(m_{y}\right)_{i, j}-\left(m_{y}\right)_{i, j-1}
\end{array}\right)=0
\end{gathered}
$$

which gives the following modified equation

$$
\begin{aligned}
\partial_{\tau} p+a_{\star}^{2} \nabla \cdot \mathbf{m}=\Delta x C_{11}\left(\partial_{x x}^{2} p+\partial_{y y}^{2} p\right)+\Delta x \mathbf{C}_{12, d=2} & \cdot\left(\begin{array}{c}
\partial_{x x}^{2} m_{x}+\partial_{y y}^{2} m_{x} \\
\partial_{x x}^{2} m_{y}+\partial_{y y}^{2} m_{y}
\end{array}\right) \\
& =\Delta x C_{11} \Delta p+\Delta x \mathbf{C}_{12, d=2} \cdot\left(\begin{array}{c}
\Delta m_{x} \\
\Delta m_{y}
\end{array}\right) .
\end{aligned}
$$

The equation on $\mathbf{m}$ of the scheme (41) with $C_{22}=0$ can be written as

$$
\begin{aligned}
\frac{\mathbf{m}_{i}^{n+1}-\mathbf{m}_{i}^{n}}{\Delta \tau}+ & \frac{1}{2 \Delta x}\left(\begin{array}{c}
p_{i+1, j}-p_{i-1, j} \\
p_{i, j+1}-p_{i, j-1}
\end{array}\right) \\
& +\frac{1}{\Delta x}\left(\begin{array}{l}
\left(\mathbf{C}_{21, d=2}\right)_{x}\left(-p_{i+1, j}+2 p_{i, j}-p_{i-1, j}-p_{i, j+1}+2 p_{i, j}-p_{i, j+1}\right) \\
\left(\mathbf{C}_{21, d=2}\right)_{y}\left(-p_{i+1, j}+2 p_{i, j}-p_{i-1, j}-p_{i, j+1}+2 p_{i, j}-p_{i, j+1}\right)
\end{array}\right)=0
\end{aligned}
$$

which gives the following modified equation

$$
\partial_{\tau} \mathbf{m}+\nabla p=\Delta x\left(\begin{array}{c}
\left(\mathbf{C}_{21, d=2}\right)_{x}\left(\partial_{x x}^{2} p+\partial_{y y}^{2} p\right) \\
\left(\mathbf{C}_{21, d=2}\right)_{y}\left(\partial_{x x}^{2} p+\partial_{y y}^{2} p\right)
\end{array}\right)=\Delta x\left(\begin{array}{c}
\left(\mathbf{C}_{21, d=2}\right)_{x} \Delta p \\
\left(\mathbf{C}_{21, d=2}\right)_{y} \Delta p
\end{array}\right) .
$$

To have diffusion on each equation, we choose $\mathbf{C}_{12, d}$ and $\mathbf{C}_{21, d}$ such that

$$
\mathbf{C}_{12, d}=C_{12, d} \mathbf{1}_{d} \quad \text { and } \quad \mathbf{C}_{21, d}=C_{21, d} \mathbf{1}_{d} \quad \text { where } \quad \mathbf{1}_{d}=\left(\begin{array}{c}
1 \\
\vdots \\
1
\end{array}\right)
$$

where $C_{12, d}$ and $C_{21, d}$ are two scalars. In the case of the dimension one, we get that $C_{12, d=1}$ and $C_{21, d=1}$ satisfy (51). However, since the diffusion depends on the dimension, we need to perform a stability analysis to get the relation between $C_{11}, C_{12, d}$ and $C_{21, d}$ where $d \geq 2$, and this is the aim of next section.

3.1.5.2 Stability analysis of the explicit new scheme in dimension two for a one dimensional flow on a regular Cartesian mesh We want to generalize the stability condition (51) to dimension two (and generalize it to dimension three). For that, we assume that we have a periodic Cartesian mesh with a characteristic length $\Delta x=\Delta y$. To simplify the study, we also assume that the flow is purely one dimensional. It means that for all $(i, j)$, we have $\left(p_{i, j}, \mathbf{m}_{i, j}\right)=$ $\left(p_{i, 1}, \mathbf{m}_{i, 1}\right)$. Then, to simplify the notation, we omit the index $j$. In dimension two, for a one dimensional flow, the new scheme can be written in the following form

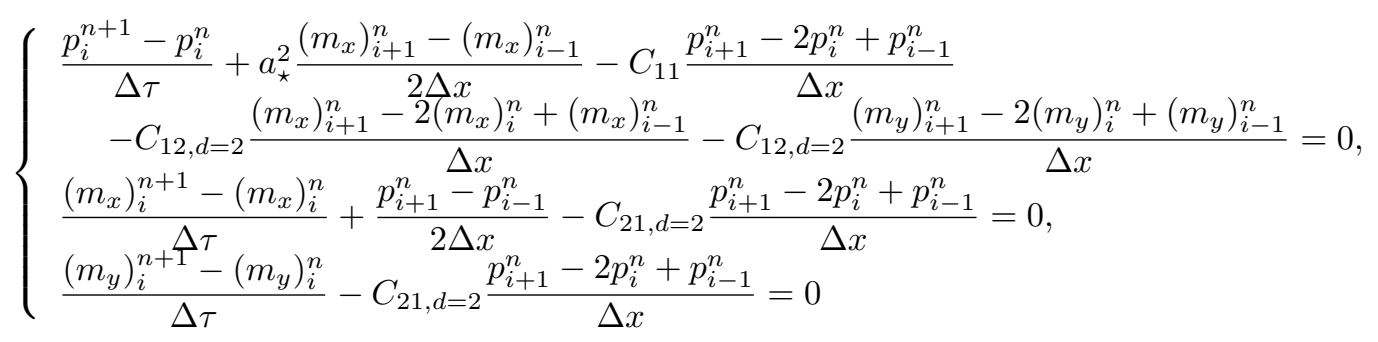


where $C_{12, d=2}=-a_{\star}^{2} C_{21, d=2}$. We want to choose $C_{11}$ and $C_{21, d=2}$ such that the scheme is stable for the same CFL condition as the explicit Roe scheme:

$$
0 \leq a_{\star} \frac{\Delta \tau}{\Delta x} \leq 1 .
$$

Proposition 3.5. The scheme (58) with $C_{12, d=2}=-a_{\star}^{2} C_{21, d=2}$ is stable under the $C F L$ condition (59) if and only if

$$
\left\{\begin{array}{l}
C_{11}=a_{\star} \\
C_{21, d=2}= \pm \frac{1}{2 \sqrt{2}} .
\end{array}\right.
$$

Proof. By substituting the discrete Fourier modes

$$
p_{i}^{n}=\varphi_{p}^{n} e^{\mathrm{j}\left(k x_{i}\right)}, \quad\left(m_{x}\right)_{i}^{n}=\varphi_{m_{x}}^{n} e^{\mathrm{j}\left(k x_{i}\right)} \quad\left(m_{y}\right)_{i}^{n}=\varphi_{m_{y}}^{n} e^{\mathrm{j}\left(k x_{i}\right)}
$$

into the fully discrete scheme, we obtain

$$
\varphi^{n+1}=A_{2} \varphi^{n} \quad \text { where } \quad \varphi=\left(\begin{array}{c}
\varphi_{p} \\
\varphi_{m_{x}} \\
\varphi_{m_{y}}
\end{array}\right)
$$

and the matrix $A_{2}=\left(\begin{array}{c}R_{1} \\ R_{2} \\ R_{3}\end{array}\right)$ is given by

$$
\begin{aligned}
& R_{1}=\left(1-4 C_{11} \frac{\Delta \tau}{\Delta x} \sin ^{2}\left(\frac{k \Delta x}{2}\right)-4 C_{12, d=2} \frac{\Delta \tau}{\Delta x} \sin ^{2}\left(\frac{k \Delta x}{2}\right)-\mathrm{j} a_{\star}^{2} \frac{\Delta \tau}{\Delta x} \sin (k \Delta x)\right. \\
& \left.-4 C_{12, d=2} \frac{\Delta \tau}{\Delta x} \sin ^{2}\left(\frac{k \Delta x}{2}\right)\right) \\
& R_{2}=\left(-4 C_{21, d=2} \frac{\Delta \tau}{\Delta x} \sin ^{2}\left(\frac{k \Delta x}{2}\right)-\mathrm{j} \frac{\Delta \tau}{\Delta x} \sin (k \Delta x) \quad 1 \quad 0\right) \\
& R_{3}=\left(\begin{array}{lll}
-4 C_{21, d=2} \frac{\Delta \tau}{\Delta x} \sin ^{2}\left(\frac{k \Delta x}{2}\right) & 0 & 1
\end{array}\right)
\end{aligned}
$$

We want a condition on $C_{11}, C_{12, d=2}$ and $C_{21, d=2}$ such that the eigenvalues of the matrix $A_{2}$ are in the unit disc. We have

$$
\begin{aligned}
\operatorname{det}\left(A_{2}-\lambda \mathrm{I}_{3}\right) & =(1-\lambda) \operatorname{det}\left(A_{1}-\lambda \mathrm{I}_{2}\right)+(1-\lambda) 16 a_{\star}^{2} C_{21, d=2}^{2}\left(\frac{\Delta \tau}{\Delta x}\right)^{2} \sin ^{4}\left(\frac{k \Delta x}{2}\right) \\
& =(1-\lambda)\left(\lambda^{2}+\gamma_{2} \lambda+\beta_{2}\right)
\end{aligned}
$$

with

$$
\begin{aligned}
& \gamma_{2}=\gamma_{1} \\
& \beta_{2}=\beta_{1}+16 a_{\star}^{2} C_{21, d=2}^{2}\left(\frac{\Delta \tau}{\Delta x}\right)^{2} \sin ^{4}\left(\frac{k \Delta x}{2}\right)
\end{aligned}
$$

where $\gamma_{1}$ and $\beta_{1}$ are given by replacing $C_{21, d=1}$ by $C_{21, d=2}$ in (52) and (53) in the proof of Proposition 3.2. We note $\lambda_{ \pm}$the two roots that can be different from 1 . In order, to ensure that the two roots are in the unit disc $\left|\lambda_{ \pm}\right| \leq 1$, the coefficients $\gamma_{2}$ and $\beta_{2}$ have to satisfy

$$
\left\{\begin{array}{l}
\beta_{2} \leq 1 \\
\left|\gamma_{2}\right| \leq 1+\beta_{2}
\end{array}\right.
$$

As in the proof of Proposition 3.2, we get

$$
\begin{aligned}
& \beta_{2} \leq 1 \\
\Leftrightarrow & \frac{\Delta \tau}{\Delta x} \sin ^{2}\left(\frac{k \Delta x}{2}\right)\left[C_{11}-a_{\star}\left(a_{\star} \frac{\Delta \tau}{\Delta x}\right)+a_{\star}^{2} \frac{\Delta \tau}{\Delta x} \sin ^{2}\left(\frac{k \Delta x}{2}\right)\left(1-8 C_{21, d=2}^{2}\right)\right] \geq 0
\end{aligned}
$$

and this inequality should be satisfied for all $0 \leq a_{\star} \Delta \tau / \Delta x \leq 1$ and for all $k \Delta x \in \mathbb{R}$. Then, we obtain

$$
\beta_{2} \leq 1 \Leftrightarrow\left\{\begin{array} { l } 
{ C _ { 1 1 } - a _ { \star } \geq 0 } \\
{ 1 - 8 C _ { 2 1 , d = 2 } ^ { 2 } \geq 0 . }
\end{array} \Leftrightarrow \left\{\begin{array}{l}
C_{11} \geq a_{\star} \\
\left|C_{21, d=2}\right| \leq \frac{1}{2 \sqrt{2}} .
\end{array}\right.\right.
$$


We also have

$$
\begin{aligned}
\left|\gamma_{2}\right| & \leq 1+\beta_{2} \\
\Rightarrow & 1 \geq\left(a_{\star} \frac{\Delta \tau}{\Delta x}\right) \sin ^{2}\left(\frac{k \Delta x}{2}\right)\left[2 \frac{C_{11}}{a_{\star}}-a_{\star} \frac{\Delta \tau}{\Delta x}+a_{\star} \frac{\Delta \tau}{\Delta x} \sin ^{2}\left(\frac{k \Delta x}{2}\right)\left(1-8 C_{21, d=2}^{2}\right)\right]
\end{aligned}
$$

for all $0 \leq a_{\star} \Delta \tau / \Delta x \leq 1$ and for all $k \Delta x \in \mathbb{R}$. and we choose the parameters $k, \Delta \tau$ and $\Delta x$ such that

$$
a_{\star} \Delta \tau / \Delta x=1 \quad \text { and } \quad \sin ^{2}\left(\frac{k \Delta x}{2}\right)=1,
$$

which gives the inequality

$$
1 \geq 2 \frac{C_{11}}{a_{\star}}-8 C_{21, d=2}^{2}
$$

By combining (62) with (61), we get

$$
\left\{\begin{array}{l}
C_{11} \leq a_{\star} \\
\left|C_{21, d=2}\right| \geq \frac{1}{2 \sqrt{2}}
\end{array}\right.
$$

Then, with (61), we obtain (60). The proof of the converse is the same as in Proposition 3.2 because we get the same roots $\lambda_{ \pm}$.

\subsubsection{Final expression of the new scheme for the wave equation}

Finally, the scheme that is accurate and stable at low Mach number under the same CFL condition as the Roe scheme consists in taking the scheme (41) with the diffusion part given by

$$
C_{11}=a_{\star}, \quad C_{22}=0, \quad \mathbf{C}_{12, d}= \pm \frac{a_{\star}^{2}}{2 \sqrt{d}} \mathbf{1}_{d} \quad \text { and } \quad \mathbf{C}_{21, d}=\mp \frac{1}{2 \sqrt{d}} \mathbf{1}_{d} \quad \text { where } \quad \mathbf{1}_{d}=\left(\begin{array}{c}
1 \\
\vdots \\
1
\end{array}\right) .
$$

The extension to dimension three can be done easily with the same study. In the next section, the aim is to extend the scheme from the wave system to the nonlinear case.

\subsection{From the linear wave equation to the barotropic Euler equations}

\subsubsection{Dimensionless wave equation}

With the previous study, since $p=\tilde{p}^{(1)} / \gamma, \mathbf{m}=(\tilde{\rho} \tilde{\mathbf{u}})^{(0)}$ and $a_{\star}=\tilde{a}^{(0)}$, we want to replace the scheme (34) by the new scheme

$$
\left\{\begin{array}{r}
\partial_{\tau} \frac{\tilde{p}_{i}^{(1)}}{\gamma}+\frac{1}{\left|\tilde{\Omega}_{i}\right|} \sum_{\Gamma_{i j} \subset \partial \Omega_{i}}\left|\tilde{\Gamma}_{i j}\right|\left[\left(\tilde{a}^{(0)}\right)^{2} \frac{(\tilde{\rho} \tilde{\mathbf{u}})_{i}^{(0)}+(\tilde{\rho} \tilde{\mathbf{u}})_{j}^{(0)}}{2} \cdot \mathbf{n}_{i j}+\tilde{a}^{(0)}\left(\frac{\tilde{p}_{i}^{(1)}}{\gamma}-\frac{\tilde{p}_{j}^{(1)}}{\gamma}\right)\right. \\
\left.\left. \pm \frac{\left(\tilde{a}^{(0)}\right)^{2}}{2 \sqrt{d}}\left((\tilde{\rho} \tilde{\mathbf{u}})_{i}^{(0)}-(\tilde{\rho} \tilde{\mathbf{u}})_{j}^{(0)}\right) \cdot \mathbf{1}_{d}\right]\right]=-\frac{1}{\gamma} \mathbf{d}_{\tilde{t}} \tilde{p}^{(0)}, \\
\partial_{\tau}(\tilde{\rho} \tilde{\mathbf{u}})_{i}^{(0)}+\frac{1}{\left|\tilde{\Omega}_{i}\right|} \sum_{\Gamma_{i j} \subset \partial \Omega_{i}}\left|\tilde{\Gamma}_{i j}\right|\left[\frac{\tilde{p}_{i}^{(1)}+\tilde{p}_{j}^{(1)}}{2 \gamma} \mathbf{n}_{i j} \mp \frac{1}{2 \sqrt{d}}\left(\frac{\tilde{p}_{i}^{(1)}}{\gamma}-\frac{\tilde{p}_{j}^{(1)}}{\gamma}\right) \mathbf{1}_{d}\right]=0 .
\end{array}\right.
$$

Dividing the first equation of $(64)$ by $\left(\tilde{a}^{(0)}\right)^{2}=\mathbf{d}_{\tilde{\rho}} \tilde{p}\left(\tilde{\rho}^{(0)}\right) / \gamma$ and noting that

$$
\mathbf{d}_{\tilde{\rho}} \tilde{p}\left(\tilde{\rho}^{(0)}\right)^{-1} \mathbf{d}_{\tilde{t}} \tilde{p}^{(0)}=\mathbf{d}_{\tilde{t}} \tilde{\rho}^{(0)}, \quad \forall i, \gamma\left(\tilde{a}^{(0)}\right)^{2} \tilde{\rho}_{i}^{(1)}=\mathbf{d}_{\tilde{\rho}} \tilde{p}\left(\tilde{\rho}^{(0)}\right) \tilde{\rho}_{i}^{(1)}=\tilde{p}_{i}^{(1)},
$$

we obtain

$$
\left\{\begin{array}{r}
\partial_{\tau} \tilde{\rho}_{i}^{(1)}+\frac{1}{\left|\tilde{\Omega}_{i}\right|} \sum_{\Gamma_{i j} \subset \partial \Omega_{i}}\left|\tilde{\Gamma}_{i j}\right|\left[\frac{(\tilde{\rho} \tilde{\mathbf{u}})_{i}^{(0)}+(\tilde{\rho} \tilde{\mathbf{u}})_{j}^{(0)}}{2} \cdot \mathbf{n}_{i j}+\tilde{a}^{(0)}\left(\tilde{\rho}_{i}^{(1)}-\tilde{\rho}_{j}^{(1)}\right)\right. \\
\left. \pm \frac{1}{2 \sqrt{d}}\left((\tilde{\rho} \tilde{\mathbf{u}})_{i}^{(0)}-(\tilde{\rho} \tilde{\mathbf{u}})_{j}^{(0)}\right) \cdot \mathbf{1}_{d}\right]=-\mathbf{d}_{\tilde{t}} \tilde{\rho}^{(0)}, \\
\partial_{\tau}(\tilde{\rho} \tilde{\mathbf{u}})_{i}^{(0)}+\frac{1}{\left|\tilde{\Omega}_{i}\right|} \sum_{\Gamma_{i j} \subset \partial \Omega_{i}}\left|\tilde{\Gamma}_{i j}\right|\left[\frac{\tilde{p}_{i}^{(1)}+\tilde{p}_{j}^{(1)}}{2 \gamma} \mathbf{n}_{i j} \mp \frac{\left(\tilde{a}^{(0)}\right)^{2}}{2 \sqrt{d}}\left(\tilde{\rho}_{i}^{(1)}-\tilde{\rho}_{j}^{(1)}\right) \mathbf{1}_{d}\right]=0 .
\end{array}\right.
$$

We remark that it could also be possible to keep $\tilde{p}_{i}^{(1)}$ instead of $\tilde{\rho}_{i}^{(1)}$. In the next section, this expression for the dimensionless wave equations is extended to the dimensionless barotropic equations. 


\subsubsection{The dimensionless new Roe scheme in the subsonic case}

We now go back to the new version of (32). The dimensionless version of the new scheme is given by

$$
\left\{\begin{aligned}
& \partial_{\tilde{t}} \tilde{\rho}_{i}+\frac{1}{M} \partial_{\tau} \tilde{\rho}_{i}+\frac{1}{\left|\tilde{\Omega}_{i}\right|} \sum_{\Gamma_{i j} \subset \partial \Omega_{i}}\left|\tilde{\Gamma}_{i j}\right|\left[\frac{\tilde{\rho}_{i} \tilde{\mathbf{u}}_{i}+\tilde{\rho}_{j} \tilde{\mathbf{u}}_{j}}{2} \cdot \mathbf{n}_{i j}\right. \\
&+M \frac{\tilde{\rho}_{i j}}{2 \tilde{a}_{i j}}\left(\tilde{\mathbf{u}}_{i j} \cdot \mathbf{n}_{i j}\right)\left(\tilde{\mathbf{u}}_{i}-\tilde{\mathbf{u}}_{j}\right) \cdot \mathbf{n}_{i j}+\frac{\tilde{a}_{i j}}{2 M}\left(\tilde{\rho}_{i}-\tilde{\rho}_{j}\right)+\frac{\tilde{a}_{i j}}{2 M}\left(1-\theta_{i j}\right)\left(\tilde{\rho}_{i}-\tilde{\rho}_{j}\right) \\
&\left.\quad \pm \frac{1}{2 \sqrt{d}}\left(1-\theta_{i j}\right)\left((\tilde{\rho} \tilde{\mathbf{u}})_{i}-(\tilde{\rho} \tilde{\mathbf{u}})_{j}\right) \cdot \mathbf{1}_{d}\right]=0 \\
& \partial_{\tilde{t}}\left(\tilde{\rho}_{i} \tilde{\mathbf{u}}_{i}\right)+\frac{1}{M} \partial_{\tau}\left(\tilde{\rho}_{i} \tilde{\mathbf{u}}_{i}\right)+ \frac{1}{\left|\tilde{\Omega}_{i}\right|} \sum_{\Gamma_{i j} \subset \partial \Omega_{i}}\left|\tilde{\Gamma}_{i j}\right|\left[\frac{\tilde{\rho}_{i}\left(\tilde{\mathbf{u}}_{i} \cdot \mathbf{n}_{i j}\right) \tilde{\mathbf{u}}_{i}+\tilde{\rho}_{j}\left(\tilde{\mathbf{u}}_{j} \cdot \mathbf{n}_{i j}\right) \tilde{\mathbf{u}}_{j}}{2}\right. \\
&+\frac{\tilde{a}_{i j}}{2 M}\left(\tilde{\rho}_{i}-\tilde{\rho}_{j}\right)\left[\tilde{\mathbf{u}}_{i j}+\left(\tilde{\mathbf{u}}_{i j} \cdot \mathbf{n}_{i j}\right) \mathbf{n}_{i j}\right] \\
&+\frac{\tilde{\rho}_{i j}}{2}\left|\tilde{\mathbf{u}}_{i j} \cdot \mathbf{n}_{i j}\right|\left(\tilde{\mathbf{u}}_{i}^{\perp}\left(\mathbf{n}_{i j}\right)-\tilde{\mathbf{u}}_{j}^{\perp}\left(\mathbf{n}_{i j}\right)\right) \\
&+M \frac{\tilde{\rho}_{i j}\left(\tilde{\mathbf{u}}_{i j} \cdot \mathbf{n}_{i j}\right)}{2 \tilde{a}_{i j}}\left[\left(\tilde{\mathbf{u}}_{i}-\tilde{\mathbf{u}}_{j}\right) \cdot \mathbf{n}_{i j}\right] \tilde{\mathbf{u}}_{i j} \\
&+\left[\frac{1}{\gamma M^{2}} \frac{\tilde{p}_{i}+\tilde{p}_{j}}{2}+\frac{\theta_{i j}}{2 M} \tilde{\rho}_{i j} \tilde{a}_{i j}\left(\tilde{\mathbf{u}}_{i}-\tilde{\mathbf{u}}_{j}\right) \cdot \mathbf{n}_{i j}\right] \mathbf{n}_{i j} \\
&\left.\mp\left(1-\theta_{i j}\right) \frac{\tilde{a}_{i j}^{2}}{2 \sqrt{d} M^{2}}\left(\tilde{\rho}_{i}-\tilde{\rho}_{j}\right) \mathbf{1}_{d}\right]=0
\end{aligned}\right.
$$

with $\theta_{i j}=\min \left(M_{i j}, 1\right)$ where

$$
M_{i j}=\max \left(M_{i}, M_{j}\right)=\max \left(\left\|\mathbf{u}_{i}\right\| / a_{i},\left\|\mathbf{u}_{j}\right\| / a_{j}\right)=M \max \left(\left\|\tilde{\mathbf{u}}_{i}\right\| / \tilde{a}_{i},\left\|\tilde{\mathbf{u}}_{j}\right\| / \tilde{a}_{j}\right) .
$$

We introduce the factor $\left(1-\theta_{i j}\right)$ behind the new terms to get the original Roe scheme if $\theta_{i j}=1$.

We quickly prove that (65) can be deduced from (66). Using the development (21) in (66), the order $M^{-1}$ gives

$$
\partial_{\tau} \tilde{\rho}_{i}^{(0)}+\frac{1}{\left|\tilde{\Omega}_{i}\right|} \sum_{\Gamma_{i j} \subset \partial \Omega_{i}}\left|\tilde{\Gamma}_{i j}\right| \frac{1+1}{2} \tilde{a}_{i j}^{(0)}\left(\tilde{\rho}_{i}^{(0)}-\tilde{\rho}_{j}^{(0)}\right)=0
$$

and under the same hypothesis of the Proposition 2.2, we get

$$
\forall \tilde{t}>0, \forall \tau>0, \forall i, \quad \tilde{\rho}_{i}^{(0)}(\tau, \tilde{t})=\tilde{\rho}_{0}^{(0)}(\tilde{t}) .
$$

The proof is similar to that of Proposition 2.2. Then the orders $M^{-1}$ and $M^{0}$ of (66) give (65).

\subsubsection{The new fix for the Roe scheme in the subsonic case expressed in original variables}

The Roe scheme corrected with the new fix is the following

$$
\left\{\begin{array}{c}
\mathbf{d}_{t} \rho_{i}+\frac{1}{\left|\Omega_{i}\right|} \sum_{\Gamma_{i j} \subset \partial \Omega_{i}}\left|\Gamma_{i j}\right|\left[\frac{\rho_{i} \mathbf{u}_{i}+\rho_{j} \mathbf{u}_{j}}{2} \cdot \mathbf{n}_{i j}+\frac{\rho_{i j}}{2 a_{i j}}\left(\mathbf{u}_{i j} \cdot \mathbf{n}_{i j}\right)\left(\mathbf{u}_{i}-\mathbf{u}_{j}\right) \cdot \mathbf{n}_{i j}\right. \\
\left.+\frac{a_{i j}}{2}\left(\rho_{i}-\rho_{j}\right)+\frac{a_{i j}}{2}\left(1-\theta_{i j}\right)\left(\rho_{i}-\rho_{j}\right) \pm \frac{1}{2 \sqrt{d}}\left(1-\theta_{i j}\right)\left((\rho \mathbf{u})_{i}-(\rho \mathbf{u})_{j}\right) \cdot \mathbf{1}_{d}\right]=0 \\
\mathbf{d}_{t}\left(\rho_{i} \mathbf{u}_{i}\right)+\frac{1}{\left|\Omega_{i}\right|} \sum_{\Gamma_{i j} \subset \partial \Omega_{i}}\left|\Gamma_{i j}\right|\left[\frac{\rho_{i}\left(\mathbf{u}_{i} \cdot \mathbf{n}_{i j}\right) \mathbf{u}_{i}+\rho_{j}\left(\mathbf{u}_{j} \cdot \mathbf{n}_{i j}\right) \mathbf{u}_{j}}{2}\right. \\
+\frac{a_{i j}}{2}\left(\rho_{i}-\rho_{j}\right)\left[\mathbf{u}_{i j}+\left(\mathbf{u}_{i j} \cdot \mathbf{n}_{i j}\right) \mathbf{n}_{i j}\right] \\
+\frac{\rho_{i j}}{2}\left|\mathbf{u}_{i j} \cdot \mathbf{n}_{i j}\right|\left(\mathbf{u}_{i}^{\perp}\left(\mathbf{n}_{i j}\right)-\mathbf{u}_{j}^{\perp}\left(\mathbf{n}_{i j}\right)\right) \\
+\frac{\rho_{i j}\left(\mathbf{u}_{i j} \cdot \mathbf{n}_{i j}\right)}{2 a_{i j}}\left[\left(\mathbf{u}_{i}-\mathbf{u}_{j}\right) \cdot \mathbf{n}_{i j}\right] \mathbf{u}_{i j} \\
+\left[\frac{p_{i}+p_{j}}{2}+\frac{\theta_{i j} \rho_{i j} a_{i j}}{2}\left(\mathbf{u}_{i}-\mathbf{u}_{j}\right) \cdot \mathbf{n}_{i j}\right] \mathbf{n}_{i j} \\
\left.\mp\left(1-\theta_{i j}\right) \frac{a_{i j}^{2}}{2 \sqrt{d}}\left(\rho_{i}-\rho_{j}\right) \mathbf{1}_{d}\right]=0
\end{array}\right.
$$


with $\theta_{i j}=\min \left(M_{i j}, 1\right)$ where $M_{i j}=\max \left(M_{i}, M_{j}\right)=\max \left(\left\|\mathbf{u}_{i}\right\| / a_{i},\left\|\mathbf{u}_{j}\right\| / a_{j}\right)$. Indeed, if considering variables (4), (14) and (20), the dimensionless version of the scheme (67) is still given by (66). We remark that we get the original Roe scheme if $\theta_{i j}=1$ in (67).

\subsubsection{Practical implementation of the new fix}

From a practical point of view, it is really easy to implement the Roe scheme corrected with the

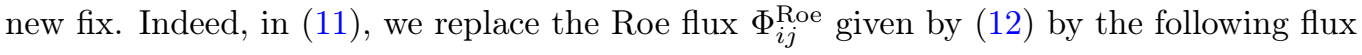

$$
\begin{aligned}
& \Phi_{i j}^{\text {New }}=\Phi_{i j}^{\text {Roe }}-\left(1-\theta_{i j}\right) \frac{\rho_{i j} a_{i j}}{2}\left(\begin{array}{c}
0 \\
\end{array}\right. {\left.\left[\left(\mathbf{u}_{i}-\mathbf{u}_{j}\right) \cdot \mathbf{n}_{i j}\right] \mathbf{n}_{i j}\right) } \\
&+\left(1-\theta_{i j}\right)\left(\begin{array}{c}
\frac{1}{2} a_{i j}\left(\rho_{i}-\rho_{j}\right) \pm \frac{1}{2 \sqrt{d}} \mathbf{1}_{d} \cdot\left(\rho_{i} \mathbf{u}_{i}-\rho_{j} \mathbf{u}_{j}\right) \\
\mp \frac{a_{i j}^{2}}{2 \sqrt{d}}\left(\rho_{i}-\rho_{j}\right) \mathbf{1}_{d}
\end{array}\right)
\end{aligned}
$$

with $\theta_{i j}=\min \left(M_{i j}, 1\right)$ and $M_{i j}=\max \left(M_{i}, M_{j}\right)=\max \left(\left\|\mathbf{u}_{i}\right\| / a_{i},\left\|\mathbf{u}_{j}\right\| / a_{j}\right)$. The first line of (68) corresponds to the correction of Dellacherie et al. and the second one is the new one to get also a scheme that is stable at low Mach number for the acoustic waves under the same CFL condition as the explicit Roe scheme.

The new fix is here applied to the Roe scheme but the same study can be done for other numerical fluxes.

\subsubsection{Implementation of wall boundary conditions}

A classical way for implementing boundary conditions, for Euler or barotropic Euler equations, i.e. the boundary conditions $\mathbf{u} \cdot \mathbf{n}=0$ along the boundary consists in using the numerical flux between the interior state and a ghost state with same thermodynamical variables, but with a ghost velocity which is the symmetric of the interior state with respect to the boundary plane. More precisely, for a given interior density $\rho_{\text {int }}$ and interior velocity $\mathbf{u}_{\text {int }}$, the ghost state is such that

$$
\left\{\begin{array}{l}
\rho_{\text {ghost }}=\rho_{\text {int }} \\
\mathbf{u}_{\text {ghost }}=\mathbf{u}_{\text {int }}-2\left(\mathbf{u}_{\text {int }} \cdot \mathbf{n}\right) \mathbf{n}
\end{array}\right.
$$

where $\mathbf{n}$ is the unit outgoing normal of the boundary. For the classical Roe scheme, this leads to the following boundary flux (using for example (12))

$$
F_{\text {boundary }}=\left(\begin{array}{c}
0 \\
p\left(\rho_{\text {int }}\right) \mathbf{n}+\rho_{\text {int }}\left(\mathbf{u}_{\text {int }} \cdot \mathbf{n}+a\left(\rho_{\text {int }}\right)\right)\left(\mathbf{u}_{\text {int }} \cdot \mathbf{n}-0\right) \mathbf{n}
\end{array}\right)
$$

This boundary condition contains a mass flux equal to 0 , and a penalization term on the normal velocity $\mathbf{u} \cdot \mathbf{n}$ in the momentum equation. If the same ghost state is used with the new scheme, the following boundary flux is found

$$
F_{\text {boundary }}=\left(\begin{array}{c} 
\pm \frac{2}{2 \sqrt{d}}\left(1-\theta_{i j}\right) \rho_{\text {int }}\left(\mathbf{u}_{\text {int }} \cdot \mathbf{n}-0\right) \mathbf{1}_{d} \cdot \mathbf{n} \\
p\left(\rho_{\text {int }}\right) \mathbf{n}+\rho_{\text {int }}\left(\mathbf{u}_{\text {int }} \cdot \mathbf{n}+\theta_{i j} a\left(\rho_{\text {int }}\right)\right)\left(\mathbf{u}_{\text {int }} \cdot \mathbf{n}-0\right) \mathbf{n}
\end{array}\right) .
$$

A penalization on the normal velocity is also found in the momentum equation, but the mass flux is nonzero if $\mathbf{u} \cdot \mathbf{n} \neq 0$, which induces mass losses in stationary computations. This mass loss on walls is unusual for Euler computations, but is rather classical for Navier-Stokes computations on imposed wall temperature [26]. Even with this mass loss, the scheme is still consistent and stable, but the convergence towards stationary problems by a time marching method may be compromised. For fixing it, the mass flux was imposed to 0 , so that the following flux is imposed at wall boundaries

$$
F_{\text {boundary }}=\left(\begin{array}{c}
0 \\
p\left(\rho_{\text {int }}\right) \mathbf{n}+\rho_{\text {int }}\left(\mathbf{u}_{\text {int }} \cdot \mathbf{n}+\theta_{i j} a\left(\rho_{\text {int }}\right)\right)\left(\mathbf{u}_{\text {int }} \cdot \mathbf{n}-0\right) \mathbf{n}
\end{array}\right) .
$$

As far as the other boundary conditions are concerned, the inlet and outlet boundary conditions are implemented with Steger-Warming like solver, as done in [16]. As the code is cell-centered, imposition of periodic boundary conditions are straightforward, by computing the numerical flux between the two cells linked by periodicity.

In the next section, the new scheme will be tested on steady low Mach number flows, and on acoustic waves propagating in low Mach number flows. 


\section{Numerical results}

The aim of this section is to bench our new scheme on both steady and unsteady problems. All the tests are performed using the AeroSol library [2] developed at INRIA.

In subsection 4.1, a numerical test is made with a one dimensional wave equation with the scheme developed in subsection 3.1. Then in subsection 4.2, the new scheme is tested with the barotropic Euler model, both with unsteady test cases including acoustic, and with steady test cases and compared with previously proposed low Mach number fixes.

\subsection{Numerical results on the linear wave equation}

We focus on the linear wave equation (38). For all numerical simulation, $a_{\star}=1$ was chosen in (38). The original Roe scheme ((39) with $\delta=1)$, is compared with the Roe scheme corrected with the Rieper or Dellacherie et al. fixes $((39)$ with $\delta=0)$ and with the Roe scheme corrected with the new fix ((41) where $C_{11}, \mathbf{C}_{21, d}, \mathbf{C}_{12, d}$ and $C_{22}$ are given by (63)), and with the exact solution.

\subsubsection{Test case}

The domain $\Omega$ is equal to $[0 ; 1]$. The numerical test corresponds to a wave which is advected at velocity $a_{\star}$. The characteristic variables are $U=\left(p-a_{\star} m\right) / 2$ which is advected at velocity $-a_{\star}$ and $V=\left(p+a_{\star} m\right) / 2$ which is advected at velocity $a_{\star}$. By taking $U(t=0, x)=0$ and

$$
V(t=0, x)=100 \sin (2 \pi \omega x),
$$

the initial condition is given by

$$
p_{0}(x)=100 \sin (2 \pi \omega x) \text { and } m_{0}(x)=p_{0}(x)
$$

where $\omega=5$. The domain is considered as periodic. The exact solution is obtained with the characteristic method. It is given by

$$
p_{\text {exact }}(x, t)=p_{0}\left(x-a_{\star} t\right) \quad \text { and } \quad m_{\text {exact }}(x, t)=p_{\text {exact }}(x, t) .
$$

All the numerical results presented below are obtained with a regular mesh $\Delta x=1 / N$ and with a time explicit solver where the time step $\Delta t$ satisfies

$$
\Delta t=\mathrm{CFL} \times \frac{\Delta x}{a_{\star}} .
$$

The CFL number will be specified in each case.

\subsubsection{Results}

We first illustrate the stability condition of the different schemes on the wave equation for an explicit time stepping. In Figure 5, we see that the Roe scheme corrected with Dellacherie et al. or Rieper fixes is stable for a CFL condition twice smaller than the Roe scheme and the Roe scheme corrected with the new fix scheme (see Proposition 2.4 and Proposition 3.2).

In Figure 6, a convergence study on the pressure variable $p$ and the momentum variable $m$ at time $t=0.3 \mathrm{~s}$ is performed with a first order finite volume computation and a second order approximation. The second order is achieved with a discontinuous Galerkin method with piecewise linear approximation in space, and a SSP integration in time [9]. The coarser and finer meshes contain $N=200$ and $N=6400$ regular cells respectively, and $\Delta x=\frac{1}{N}$. The right order of accuracy is observed with the finite volume approximation with all fixes. With the second order approximation, the Roe scheme corrected with Dellacherie et al. or Rieper fixes is stable under a CFL condition approximately ten times smaller than the one of the Roe scheme and the Roe scheme corrected by the new fix [14]. Moreover, only a first order of accuracy is observed for the momentum with the Dellacherie et al. or Rieper fixes. With the new scheme, we get the right order of accuracy 2 on both variables.

\subsection{Numerical results on the barotropic Euler equation}

We present some numerical results on the barotropic Euler equations (1). For all numerical application, the equation of state is given by (2) with $\kappa=1$ and $\gamma=2$. The behavior of the Roe scheme, the Roe scheme corrected with the Rieper, the Dellacherie et al. or the new fix on a low Mach number acoustic wave (test case of subsubsection 2.2.1) in the one dimensional case are studied. Then results obtained on a classical steady low Mach number test case are given, which proves that the scheme is accurate for both steady and unsteady computations at low Mach number. 

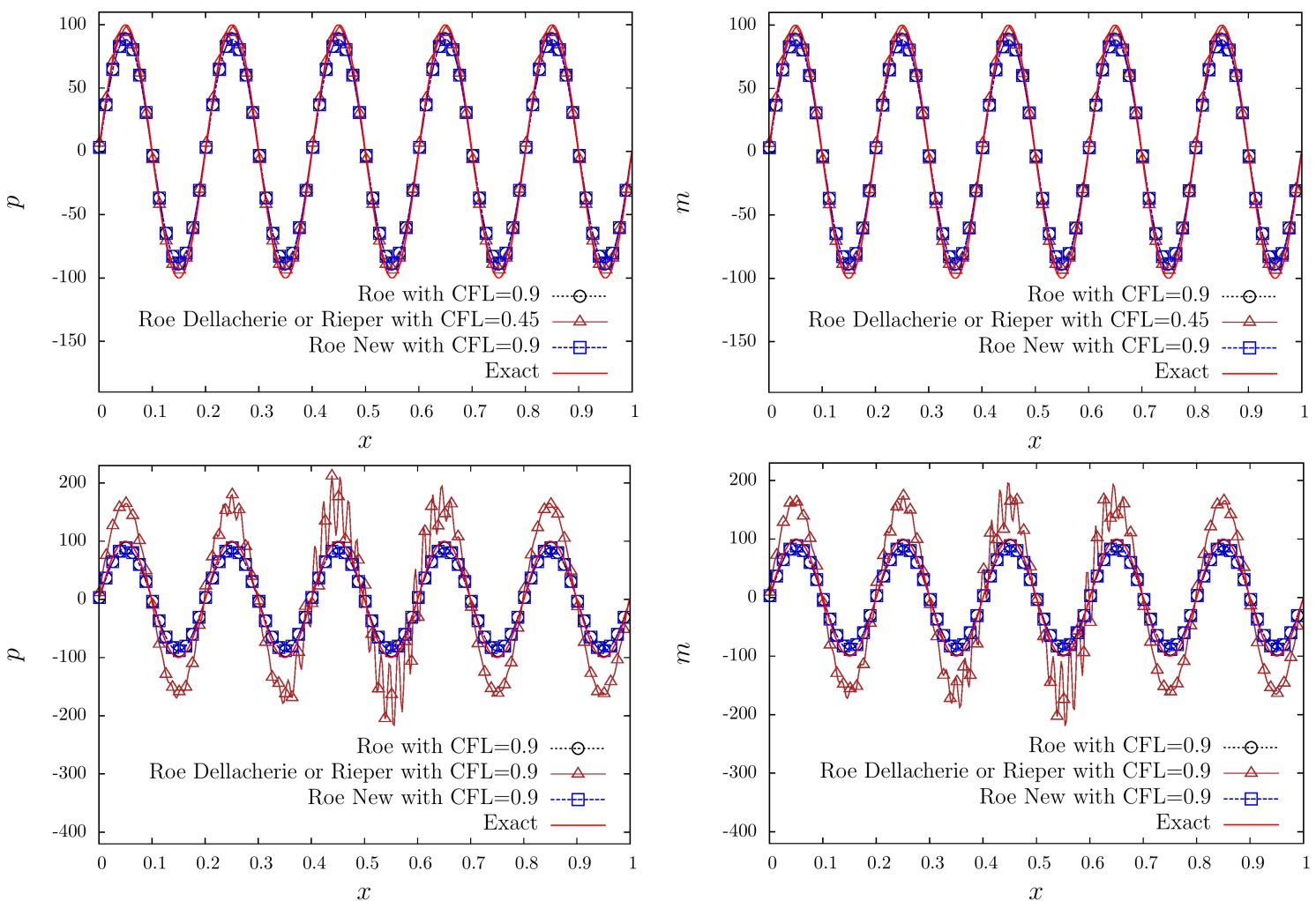

Figure 5: Pressure $p$ (left) and momentum $m$ (right) obtained at time $t=1 \mathrm{~s}$ on the sinusoidal test case described in subsubsection 4.1.1 (linear wave equation) with $N=400$ regular cells and with a first order approximation (explicit time stepping) with a CFL number of 0.9 (bottom) and a CFL number of 0.45 (top).
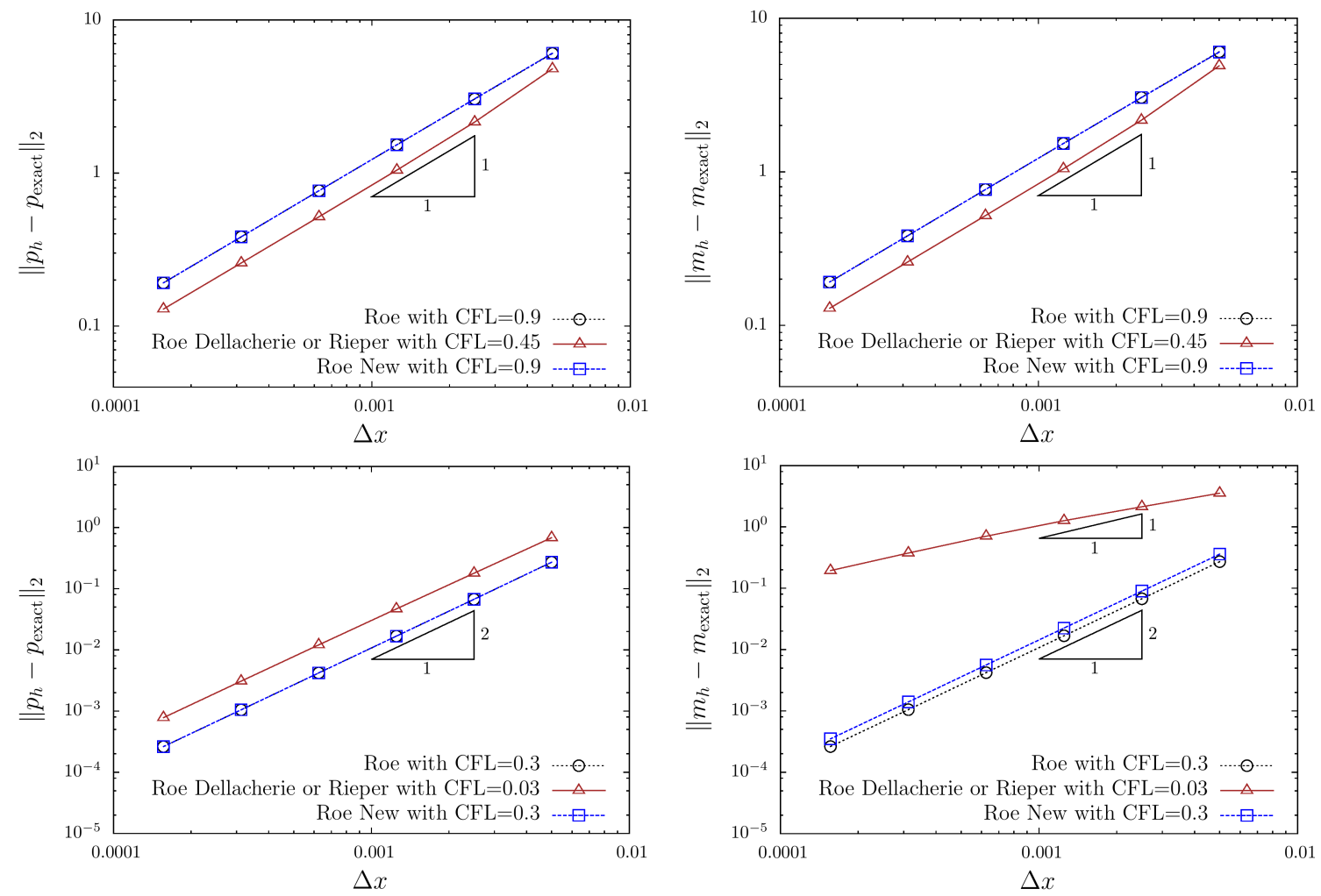

Figure 6: Order obtained on the pressure (left) and on the momentum (right) on the sinusoidal test case described in subsubsection 4.1.1 (linear wave equation) at time $t=0.3 \mathrm{~s}$ with the Roe scheme, and different fixes with a first (top) and a second order (bottom) approximations. 


\subsubsection{Computation of an acoustic wave in a one dimensional low Mach number flow}

In Figure 7, results obtained with the first order new scheme (explicit time stepping) on the one dimensional propagation of an acoustic wave are shown. Stability is observed for a CFL number comparable with the one of the original Roe scheme.
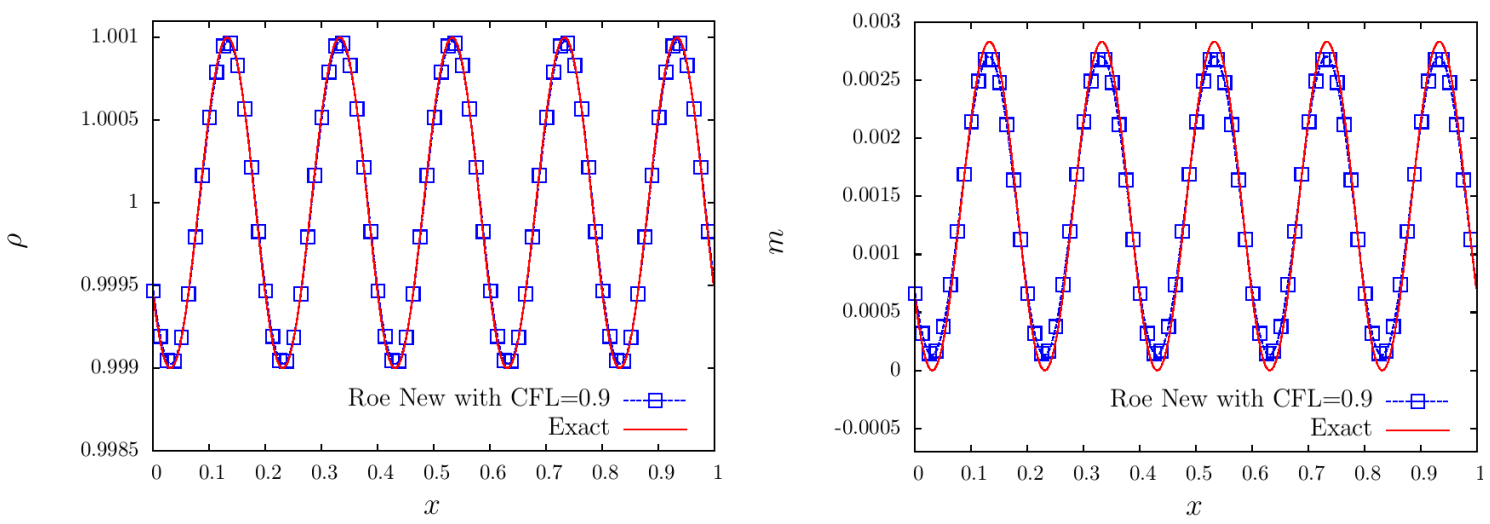

Figure 7: Density $\rho$ (left) and momentum $m$ (right) obtained at time $t=0.3408 \mathrm{~s}$ on the sinusoidal test case described in subsubsection 2.2 .1 with $N=400$ regular cells and with a first order approximation (explicit time stepping) with a CFL number of 0.9 .

In Figure 8, a convergence study of the $L^{2}$ norm of the density $\rho$ and the momentum $m$ at time $t=0.3408 \mathrm{~s}$ is performed. The coarser and finer meshes contain $N=200$ and $N=6400$ regular cells, respectively, and $\Delta x=\frac{1}{N}$. First and second order accuracy are tested. Curves prove that, with the new fix, the optimal order is obtained in both the first and second order appoximations. This is an improvement with respect to the results obtained in Figure 4, where the optimal order was not reached with the Rieper and Dellacherie et al. low Mach number fixes, and with the results of Figure 1, which proved that the Roe-Turkel scheme is not able to accurately compute the propagation of an acoustic wave in a low Mach number flow.

\subsubsection{Computation of an acoustic wave in a two dimensional low Mach number flow}

In this test, a propagating acoustic wave in a two dimensional steady low Mach flow is considered. As in [35], the initial condition is a superposition of a stationary low Mach flow and an acoustic perturbation and is given by

$$
\left\{\begin{array}{l}
\rho_{0}=\rho_{0}^{\text {Stationary }}+\rho_{0}^{\text {Acoustic }} \\
\mathbf{u}_{0}=\mathbf{u}_{0}^{\text {Stationary }}+\mathbf{u}_{0}^{\text {Acoustic }}
\end{array}\right.
$$

where $\left(\rho_{0}^{\text {Stationary }}, \mathbf{u}_{0}^{\text {Stationary }}\right)$ matches with the stationary vortex and $\left(\rho_{0}^{\text {Acoustic }}, \mathbf{u}_{0}^{\text {Acoustic }}\right)$ with the acoustic perturbation. The computational domain is $[-1 ; 1] \times[0 ; 1]$.

Still following [35], the stationary part of the flow is a Gresho vortex [20], which is an incompressible rotating flow centered in $(0,0.5)$. The initial angular velocity $\left(u_{0}^{\phi}\right)^{\text {Stationary }}$ is given by

$$
\left(u_{0}^{\phi}\right)^{\text {Stationary }}(r)= \begin{cases}5 r, & \text { if } 0 \leq r<0.2, \\ 2-5 r, & \text { if } 0.2 \leq r<0.4, \\ 0, & \text { if } 0.4 \leq r\end{cases}
$$

where $r=\sqrt{x^{2}+(y-0.5)^{2}}$ denotes the radial coordinate. The initial pressure $p_{0}^{\text {Stationary }}$ is given by

$$
p_{0}^{\text {Stationary }}(r)= \begin{cases}p_{c}+\frac{25}{2} r^{2}, & \text { if } 0 \leq r<0.2, \\ p_{c}+\frac{25}{2} r^{2}+4(1-5 r-\ln (0.2)+\ln (r)), & \text { if } 0.2 \leq r<0.4, \\ p_{c}-2+4 \ln (2), & \text { if } 0.4 \leq r,\end{cases}
$$

where $p_{c}$ is the pressure in the vortex center. We choose $p_{c}=\kappa\left(1 /\left(\kappa \gamma M^{2}\right)\right)^{\frac{\gamma}{\gamma-1}}-\frac{1}{2}$ such that the maximal Mach number of the flow is $M$. Indeed, the velocity reaches its maximum values $\left(u_{0}^{\Phi}\right)_{\max }^{\text {Stationary }}=1$ at $r=0.2$ and we have $p_{0}^{\text {Stationary }}(r=0.2)=p_{c}+\frac{1}{2}$.

The acoustic part of the flow is a plane acoustic wave propagating from the left to the right, defined as

$$
\rho_{0}^{\text {Acoustic }}(x, y)= \begin{cases}\rho_{\infty} M \cos (5 \pi(x+0.7)), & \text { if }-0.8<x<-0.6 \\ 0, & \text { elsewhere }\end{cases}
$$



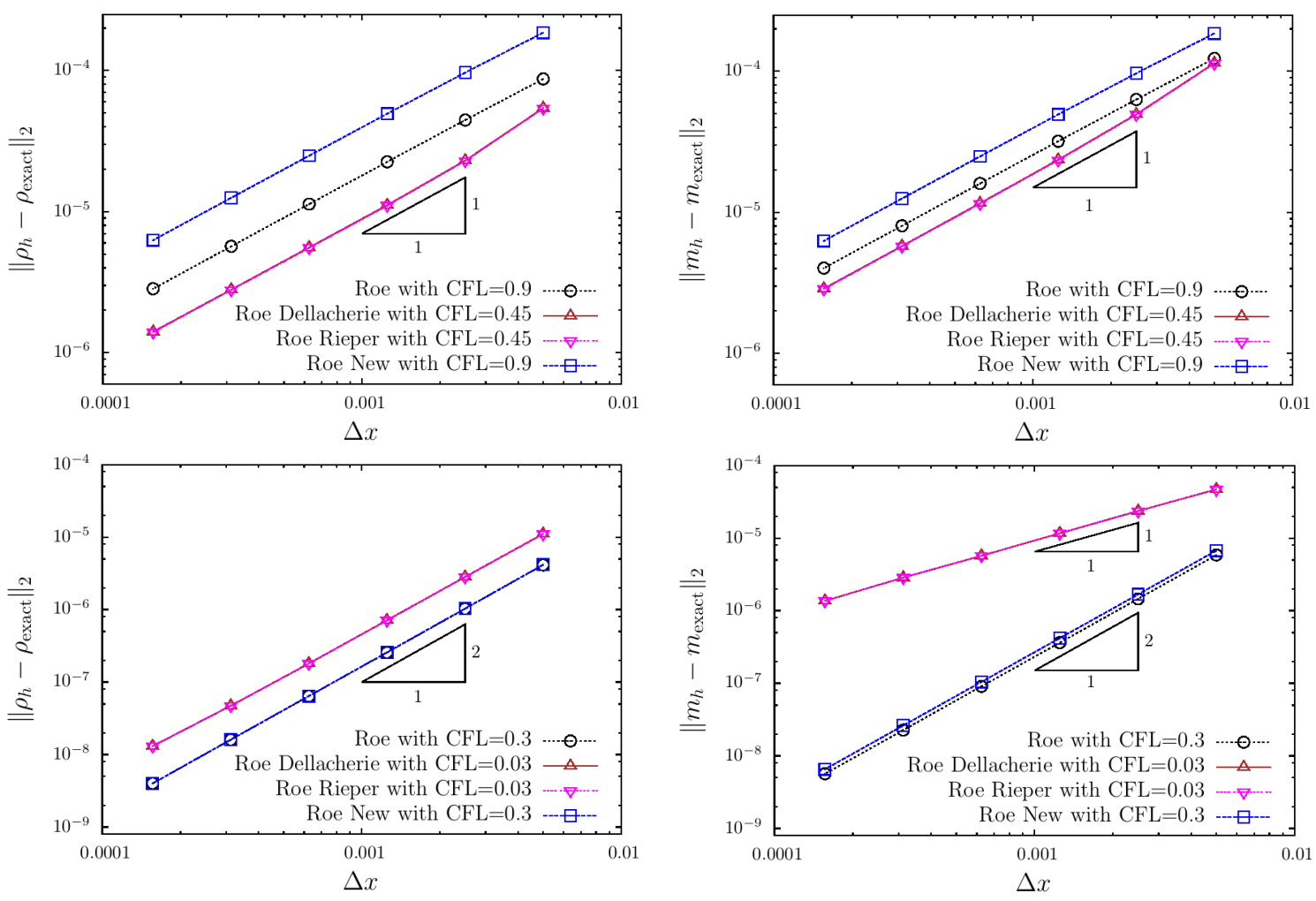

Figure 8: Order obtained on the density (left) and on the momentum (right) on the sinusoidal test case described in subsubsection 2.2.1 at time $t=0.3408 \mathrm{~s}$ with the Roe scheme and different fixes with a first (top) and a second order (bottom) approximations.

where $\rho_{\infty}=\rho\left(p_{\infty}\right)$ with $p_{\infty}=p_{c}-2+4 \ln (2)$ and

$$
\begin{aligned}
& \left(u_{x}\right)_{0}^{\text {Acoustic }}(x, y)=\frac{2}{\gamma-1}\left(a\left(\rho_{\infty}+\rho_{0}^{\text {Acoustic }}(x, y)\right)-a_{\infty}\right) \\
& \left(u_{y}\right)_{0}^{\text {Acoustic }}(x, y)=0 .
\end{aligned}
$$

The top and bottom boundary conditions of the domain are periodic, while inlet and outlet boundary conditions are used on the left and on the right for ensuring that the acoustic wave leaves the domain.

For such a low Mach number base flow, the acoustic wave is expected to propagate to the right, pass through the vortex with a weak interaction (since the acoustic and incompressible parts of the flow are nearly decoupled in such a low Mach number limit $[49,11])$ to finally leave the domain.

Results shown were obtained with $M=10^{-3}$. In Figure 9, the Mach number field obtained on a $800 \times 400$ Cartesian grid with the Roe scheme, the new scheme and the Roe-Turkel scheme at different times is shown. With the Roe scheme and the new scheme, the acoustic wave goes through the vortex without interaction. At time $t=2 \times 10^{-2} \mathrm{~s}$, when the acoustic wave has left the domain for a long time, the vortex is completely dissipated with the Roe scheme while it is well preserved with the new scheme. As far as the Roe-Turkel scheme is concerned, the acoustic wave does not propagate at all and is dissipated in situ, in agreement with results of subsubsection 2.3.1.

In Figure 10, the Mach number field obtained with an unstructured grid containing 845910 triangular cells with the Roe scheme and the new scheme is shown. Good results are obtained with both schemes as the sound wave propagates through the vortex which is well preserved over time with both schemes, as expected. So the use of the new scheme with an unstructured mesh permits to recover a correct acoustic wave propagation.

\subsubsection{Computation of a steady low Mach number flow}

In the previous section, the new scheme was proven to be more accurate than the previous low Mach number fixes for computing the propagation of acoustic waves in a low Mach number flow. The aim of this section is to prove that the new scheme is also able to compute accurately steady low Mach number flows. The scattering of a flow by a cylinder is selected as a test case. 


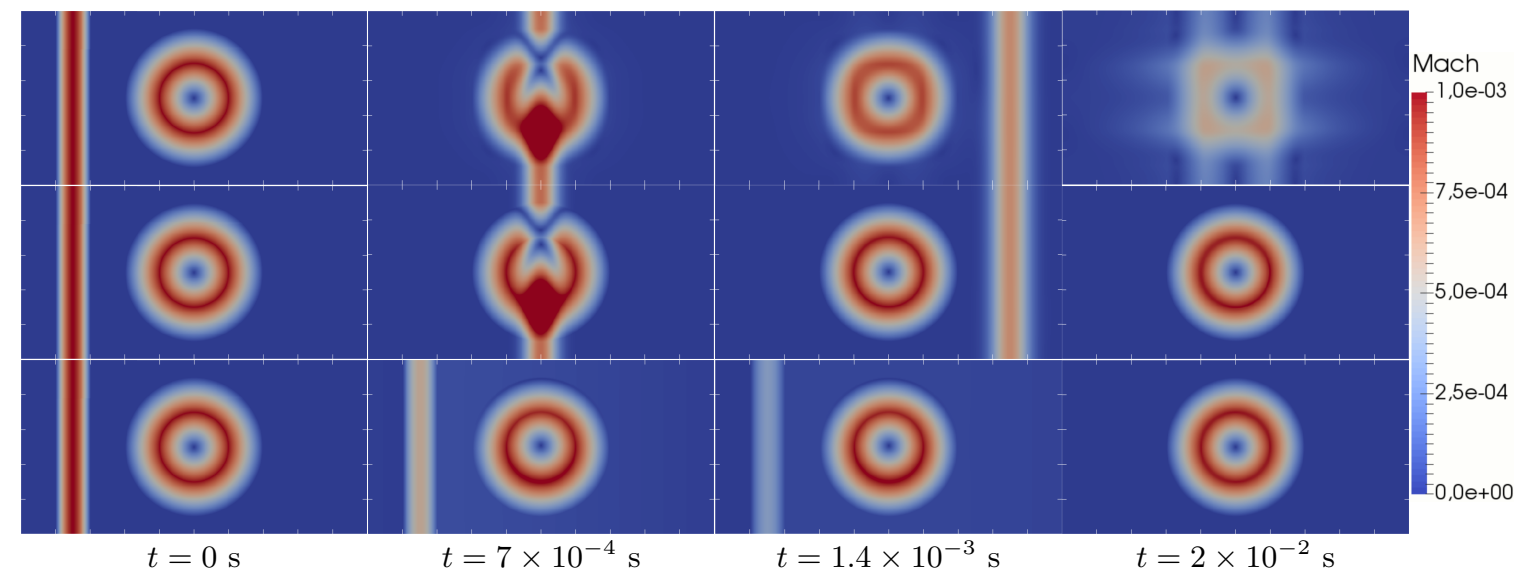

Figure 9: Mach number obtained at time $t=0 \mathrm{~s}$ (left), $t=7 \times 10^{-4} \mathrm{~s}$ (center left), $t=1.4 \times 10^{-3} \mathrm{~s}$ (center right) and $t=2 \times 10^{-2} \mathrm{~s}$ with a $800 \times 400$ Cartesian grid on the test case described in subsubsection 4.2.2 for the Roe scheme (top), the new scheme (middle) and the Roe-Turkel scheme (bottom).

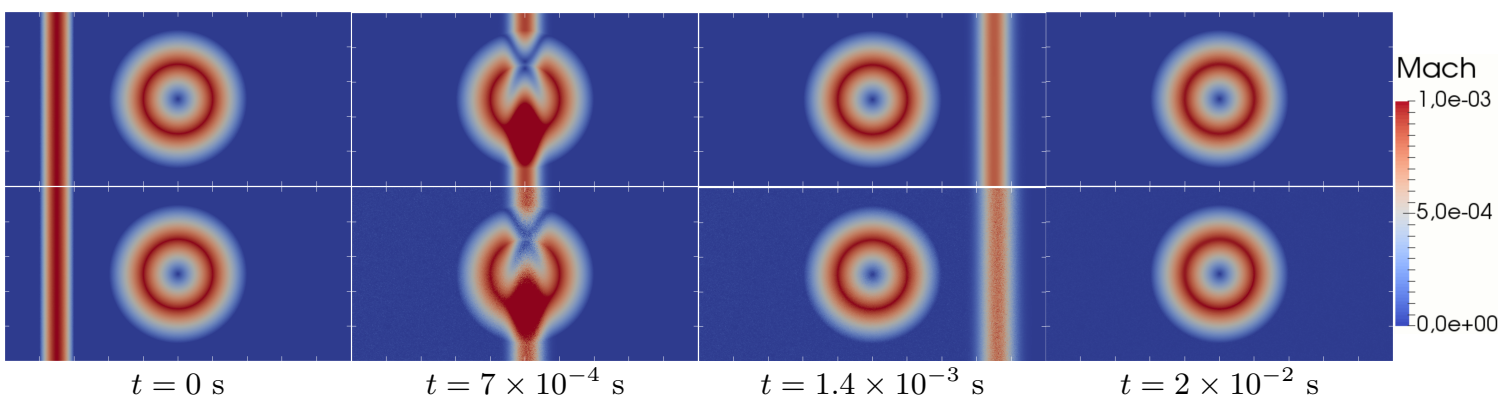

Figure 10: Mach number obtained at time $t=0 \mathrm{~s}$ (left), $t=7 \times 10^{-4} \mathrm{~s}$ (center left), $t=1.4 \times 10^{-3} \mathrm{~s}$ (center right) and $t=2 \times 10^{-2} \mathrm{~s}$ with a unstructured triangular mesh on the test case described in subsubsection 4.2.2 for the Roe scheme (top) and the new scheme (bottom). 
4.2.3.1 Test case description We are interested in the scattering of a low Mach number flow by a cylinder of radius $r_{0}$. This test is useful since an analytical reference solution is known. The domain $\Omega$ is an annulus $\left[r_{0}, r_{1}\right] \times\left[0,2 \pi\right.$. Here, we used $r_{0}=0.5$ and $r_{1}=5.5$. The initial data are uniform and set equal to

$$
\rho_{0}=1, \quad \mathbf{u}_{0}=\left(u_{0}, 0\right)^{T},
$$

with $u_{0}=a_{0} M_{\infty}$ where $a_{0}=\sqrt{\mathbf{d}_{\rho} p\left(\rho_{0}\right)}$ and $M_{\infty}$ is the Mach number at infinity. The exact solution at infinity is uniform and given by

$$
\rho_{\infty}=1, \quad \text { and } \quad \mathbf{u}_{\infty}=\left(u_{0}, 0\right)^{T} .
$$

We consider wall boundary condition on the internal cylinder of radius $r_{0}$ and inlet or outlet boundary condition on the external cylinder on radius $r_{1}$. For the numerical application, the equation of state is given by (2) with $\kappa=1$ and $\gamma=2$. We will present numerical results on triangular and quadrangular meshes.

4.2.3.2 Reference solution We want a stationary solution of (1) with $\mathbf{u} \cdot \mathbf{n}=0$ on the cylinder and $\rho=\rho_{\infty}$ and $\mathbf{u}=\mathbf{u}_{\infty}$ at infinity. The dimensionless solution is then the stationary solution of (5) with $\tilde{\mathbf{u}} \cdot \mathbf{n}=0$ on the cylinder and $\tilde{\rho}_{\infty}=1$ and $\tilde{\mathbf{u}}_{\infty}=(1,0)^{T}$ at infinity. The exact solution, developed in power of the Mach number is (see [14, 27] for computation details)

$$
\left\{\begin{array}{l}
p_{\text {ref }}(r, \theta)=p_{\infty}+\frac{p_{\infty}}{2}\left(2 \frac{r_{0}^{2}}{r^{2}} \cos (2 \theta)-\frac{r_{0}^{4}}{r^{4}}\right) M^{2}+\mathcal{O}\left(M^{3}\right) \\
\left(u_{x}\right)_{\mathrm{ref}}(r, \theta)=u_{\infty}-u_{\infty} \frac{r_{0}^{2}}{r^{2}} \cos (2 \theta)+\mathcal{O}(M), \\
\left(u_{y}\right)_{\mathrm{ref}}(r, \theta)=-u_{\infty} \frac{r_{0}^{2}}{r^{2}} \sin (2 \theta)+\mathcal{O}(M) .
\end{array}\right.
$$

4.2.3.3 Numerical results The annulus $\left[r_{0}, r_{1}\right] \times[0,2 \pi[$ is discretized with a quadrangular mesh with a resolution of $n_{r}=50$ in the radial direction and $n_{\theta}=160$ in the orthoradial direction, corresponding to a total of 8000 cells. The annulus is also discretized with a triangular mesh obtained with GMSH [18], with a characteristic length on the internal circle of $l c_{0}=0.04$ and $l c_{1}=0.43$ on the external circle. The mesh contains 5154 triangular cells.

Numerical results are obtained with a time explicit solver and a CFL number equal to 0.4, except for the Roe-Turkel scheme (see subsubsection 1.4.3) for which we use an implicit solver because of the CFL restriction and a fixed time step equal to $\Delta t=5 \times 10^{-3} / M_{\infty}$. The final time to capture the stationary solution is $t_{\max }=50 \mathrm{~s}$ except for the Roe-Turkel scheme for which we use $t_{\max }=\max \left(50,2 / M_{\infty}\right) \mathrm{s}$.

Isolines of the pressure fluctuations $p_{\text {ref }}-p_{\infty}$ obtained for the stationary solution with a Mach number $M_{\infty}$ equal to $10^{-3}$ are shown in Figure 11 with the quadrangular mesh and in Figure 12 with the triangular mesh. On the triangular mesh, the Roe scheme and the different fixes give a good approximation of the incompressible analytical solution. On the quadrangular mesh, the pressure fluctuations show one order of magnitude higher than the incompressible analytical solution with the Roe scheme, whereas a solution similar to the analytical incompressible solution is observed with the different fixes, including the new one.

Results shown in Figure 11 and Figure 12 for the new fix do not look symmetric with respect to the axis $y=0$. This is due to the fact that the new numerical flux is not Galilean invariant (see Remark 3.3). For magnifying this, isolines obtained on the top of the domain have been superposed with the isolines of the bottom of the domain in Figure 13. The asymmetry of the new scheme is larger than the original Roe scheme on triangles, and than the Roe scheme with Dellacherie et al. fix on quadrangles.

Last, we numerically study with the new fix the variation of $\left\|\tilde{\rho}_{h}-\tilde{\rho}_{\infty}\right\|_{2}$ as a function of the Mach number on a triangular and a quadrangular meshes. The Mach number at infinity varies from $10^{-1}$ to $10^{-4}$. The initial condition is still given by equation (69), with $M_{\infty} \in\left\{10^{-1}, 10^{-2}, 10^{-3}, 10^{-4}\right\}$. The results are presented in Figure 14. The behaviour consistent with the continuous asymptotic expansion, namely $\left\|\tilde{\rho}_{h}-\tilde{\rho}_{\infty}\right\|_{2}=\mathcal{O}\left(M^{2}\right)$ is observed for all the numerical schemes with the triangular mesh. On the quadrangular mesh, the $\mathcal{O}(M)$ behaviour is observed for the Roe scheme, whereas with the low Mach number fixes, including the new one, the $\mathcal{O}\left(M^{2}\right)$ behaviour is recovered.

\subsubsection{Transonic airfoil}

We consider a transonic flow to test the new scheme for a flow Mach number close to 1. Indeed, since the new scheme reverts to the Roe scheme for Mach number greater than one and is dif- 


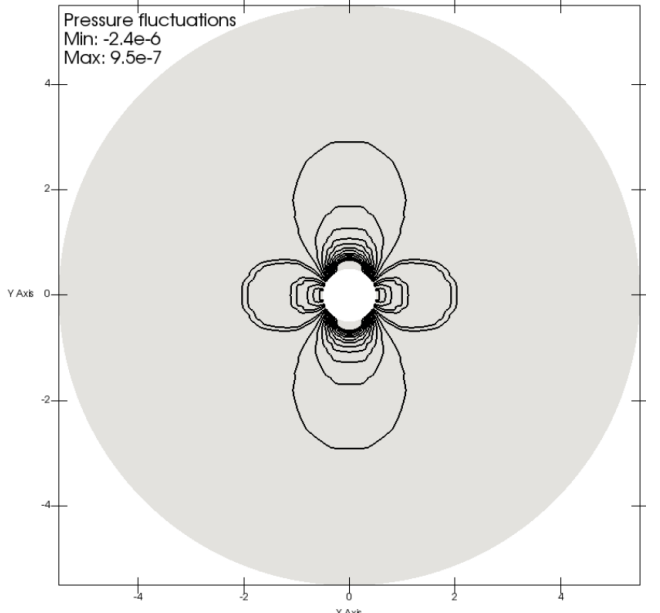

Incompressible analytical
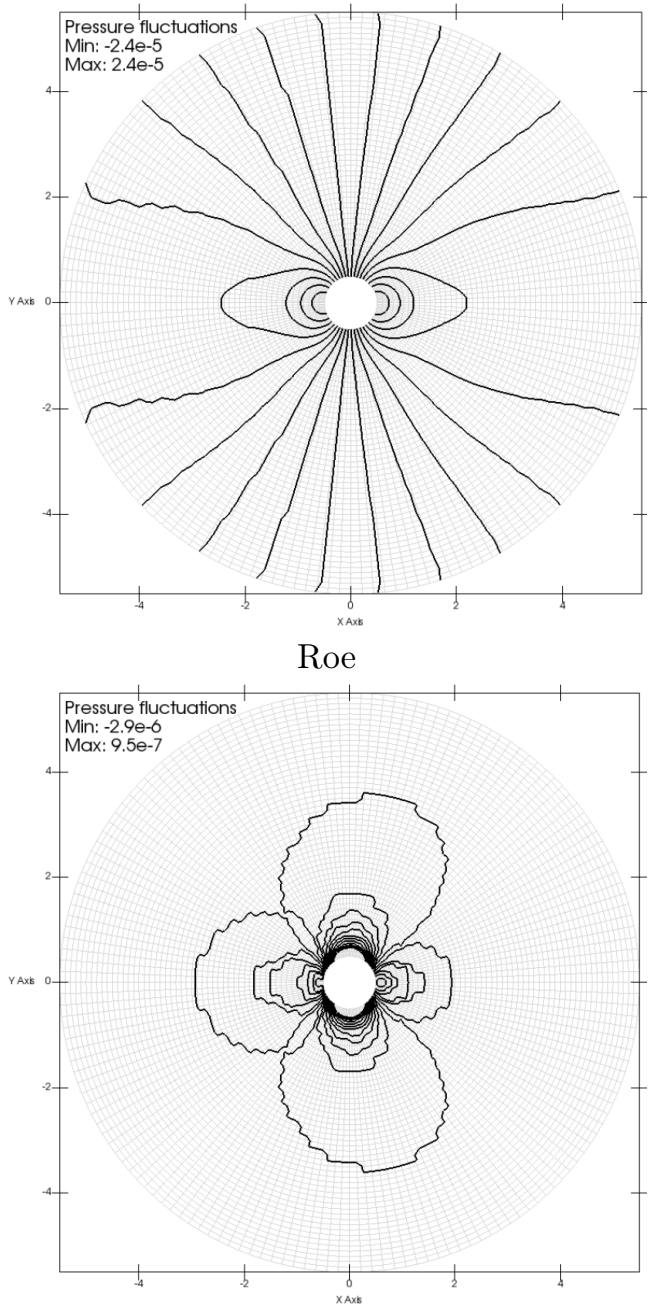

Roe with Dellacherie and al. fix
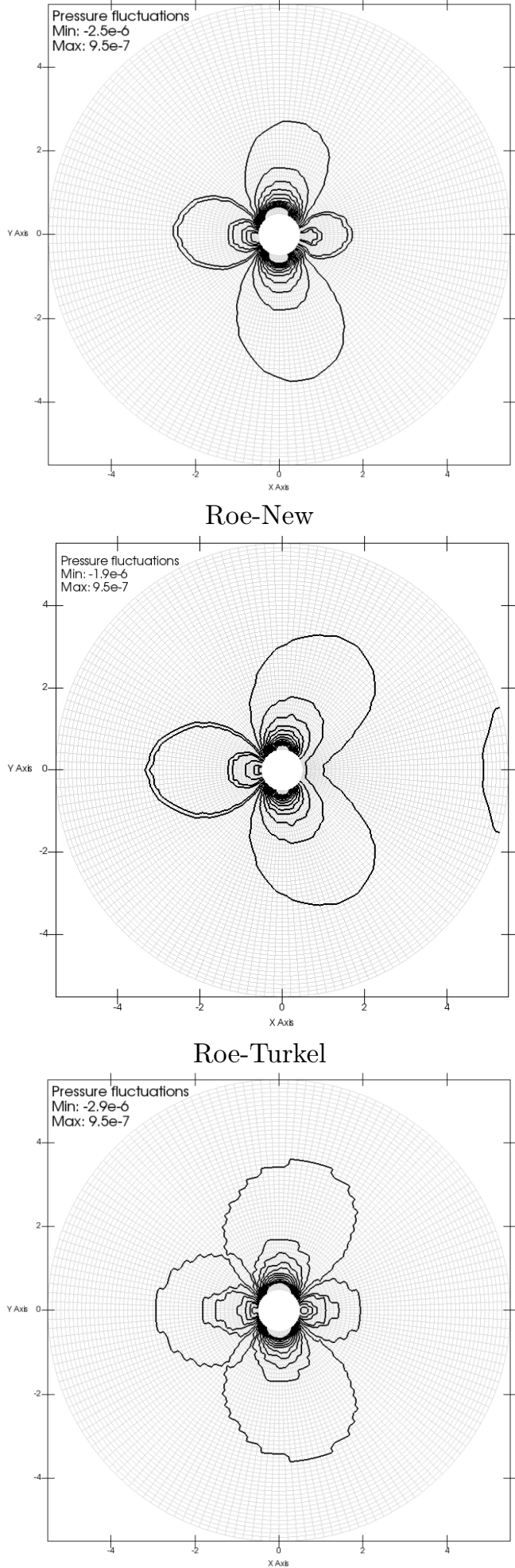

Roe with Rieper fix

Figure 11: Isolines of the pressure fluctuations $p-p_{\infty}$ obtained at $M_{\infty}=10^{-3}$ with a quadrangular mesh on the test case of the scattering of a flow by a cylinder for the Roe scheme and different classical low Mach number fixes. 20 isolines were drawn, between $-2.4 \times 10^{-5}$ and $2.4 \times 10^{-5}$ for the original Roe scheme, and 20 isolines were drawn, between $-1.5 \times 10^{-6}$ and $10^{-6}$ for the other results. 

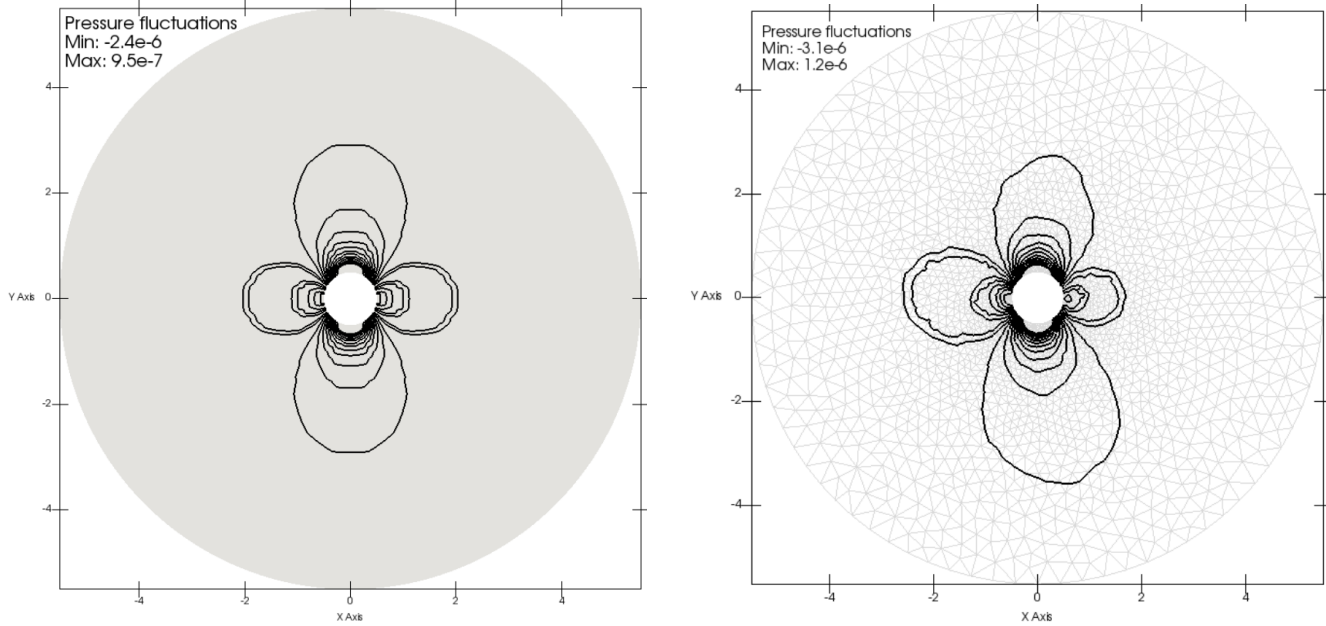

Incompressible analytical
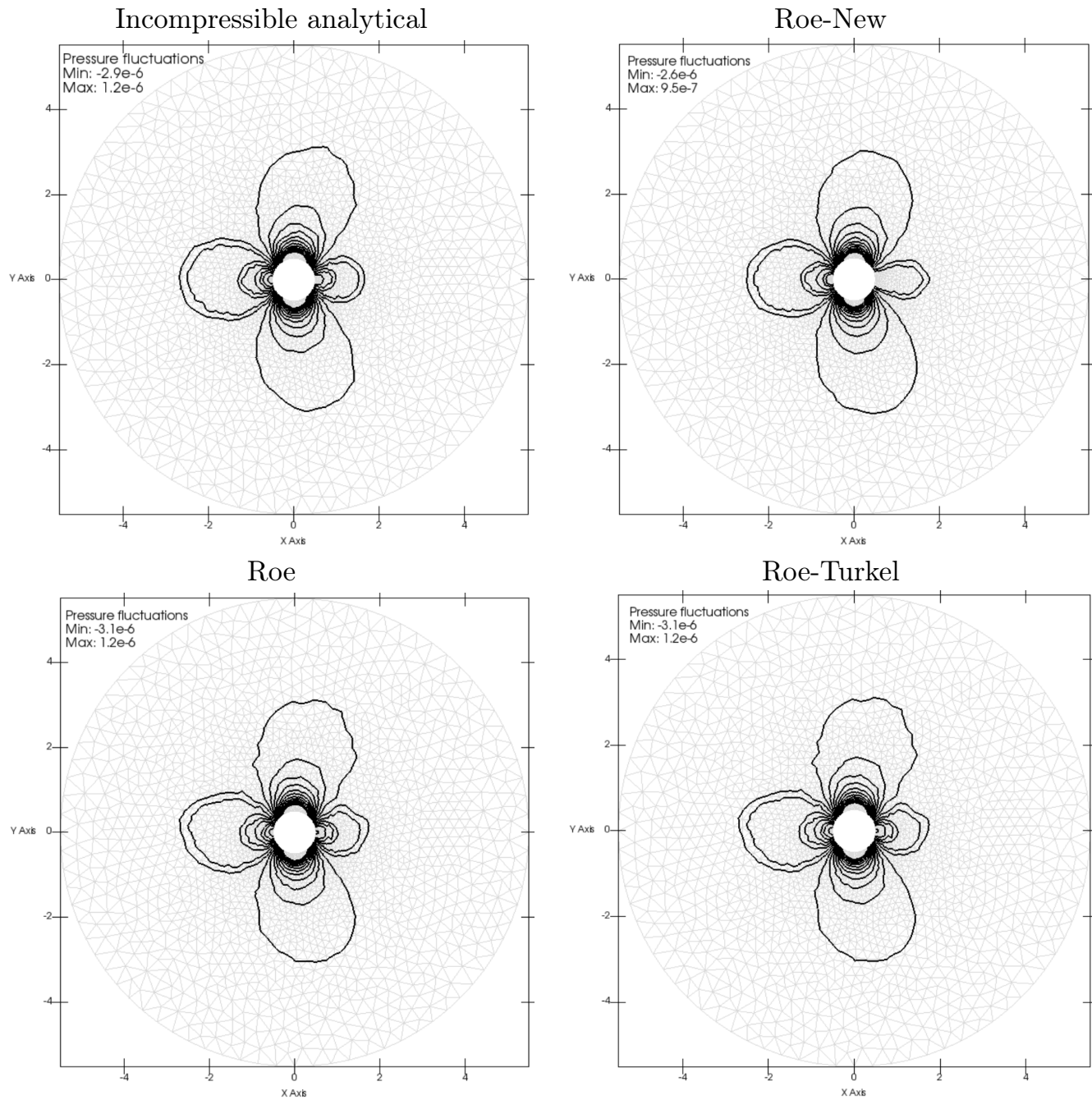

Roe with Dellacherie and al. fix

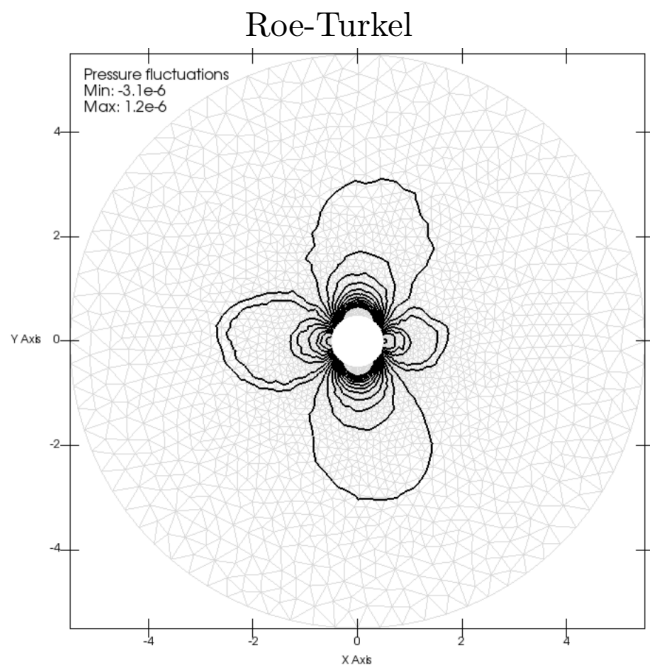

Roe with Rieper fix

Figure 12: Isolines of the pressure fluctuations $p-p_{\infty}$ obtained at $M_{\infty}=10^{-3}$ with a triangular mesh on the test case of the scattering of a flow by a cylinder for the Roe scheme and different classical low Mach number fixes. 20 isolines were drawn, between $-1.5 \times 10^{-6}$ and $10^{-6}$ for all schemes. 


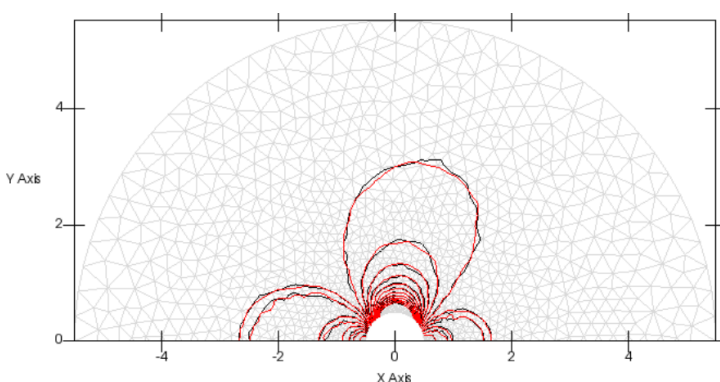

Roe (triangles)

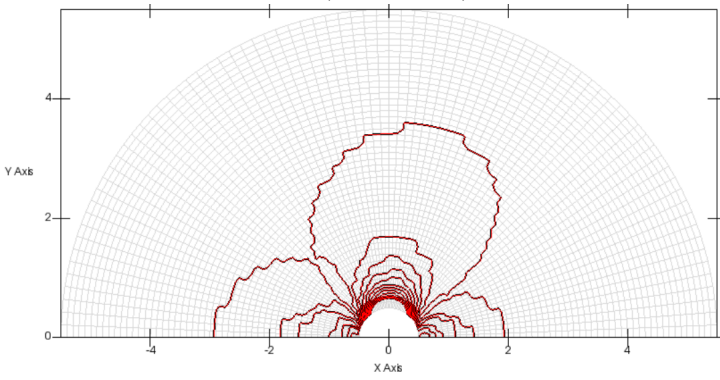

Roe with Dellacherie and al. fix (quads)

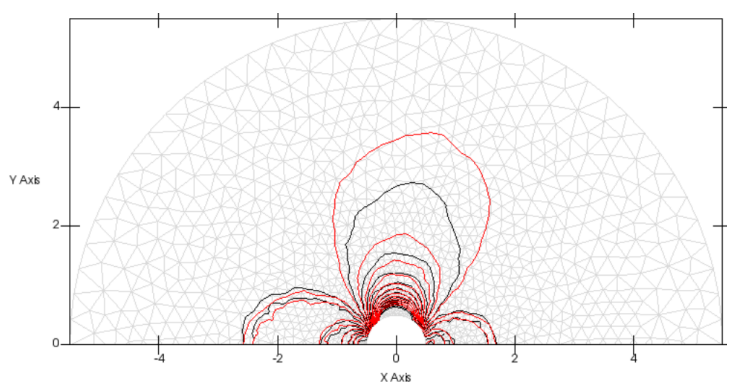

Roe with the new fix (triangles)

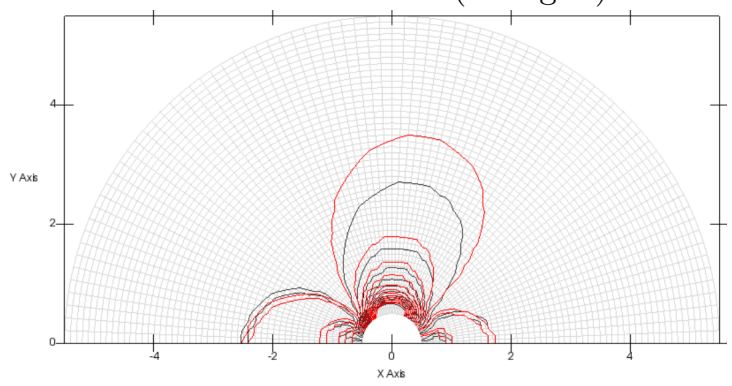

Roe with the new fix (quads)

Figure 13: Superposition of the isolines of the pressure fluctuations of the top (black lines) and the bottom domain (red lines), obtained on an unstructured triangular mesh (top) and on a quadrangular mesh (bottom).
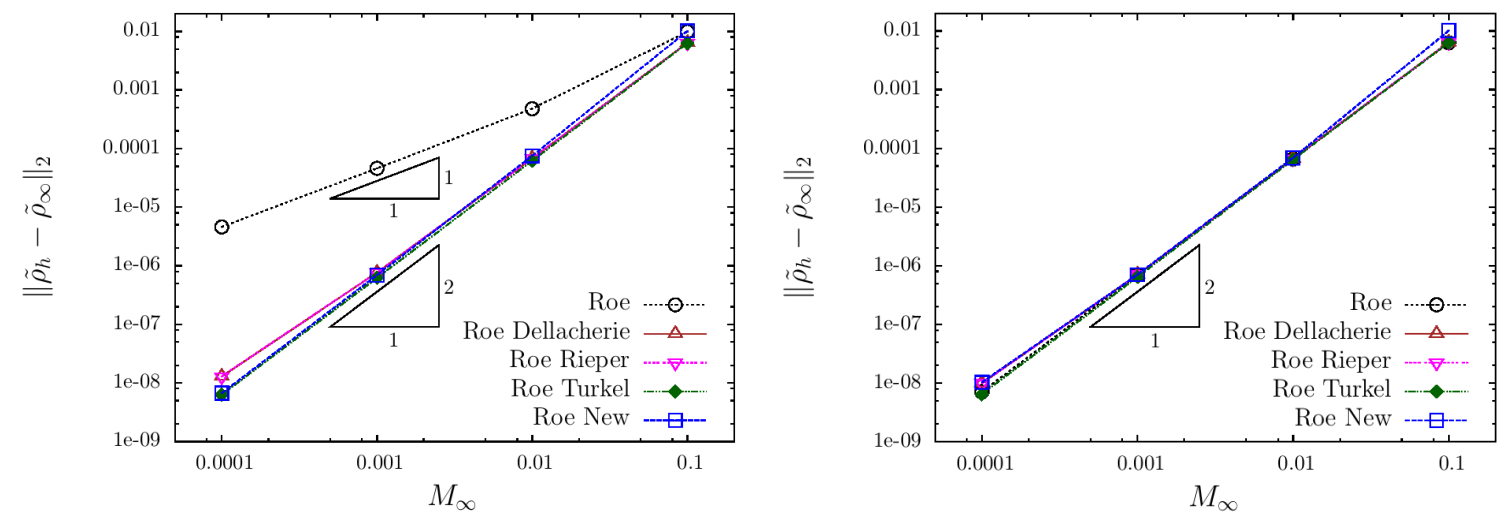

Figure 14: $L^{2}$ norm of the difference $\tilde{\rho}_{h}-\tilde{\rho}_{\infty}$ obtained for Mach number at infinity $M_{\infty}$ varying from $10^{-1}$ to $10^{-4}$ with a quadrangular mesh (left) and with a triangular mesh (right) on the test case of the scattering of a flow by a cylinder for the Roe scheme and different classical low Mach number fixes. 
ferent for Mach number smaller than one (see subsubsection 3.2.4), it is interesting to study the behaviour of the scheme for Mach number close to one. We consider the transonic flow around a NACA0012 airfoil with an incoming Mach number of 0.75 at an angle of attack of $4^{\circ}$ [34]. The Mach number of the resulting stationary flow varies between 0.05 and 1.45 . The domain $\Omega$ is the square $[-0.75,1.75] \times[-1,1]$ with the NACA airfoil located in the middle. An unstructured mesh containing 48179 quadrangular cells is used. Wall boundary condition on the airfoil and inlet or outlet boundary condition on the external border are prescribed. The initial data fields are uniform and set equal to

$$
\rho_{0}=1, \quad \mathbf{u}_{0}=\left(u_{0} \cos \theta, u_{0} \sin \theta\right)^{T}
$$

where $\theta=4^{\circ}, u_{0}=a_{0} M_{\infty}$ where $a_{0}=a\left(\rho_{0}\right)$ and $M_{\infty}$ is the Mach number at infinity, here set to 0.75 . From a numerical point of view, the final time to capture the stationary solution is $t_{\max }=50 \mathrm{~s}$.

In Figure 15, the Mach number isolines obtained with the Roe scheme (black) and with the Roe scheme corrected with the new fix (blue) are plotted. 20 isolines were drawn, between 0.1 and 1.4. Only marginal differences are observed between the results provided by the two schemes. The new scheme also captures equally well the shock wave at the top of the aircraft, which proves that it has a good behaviour for such a transonic flow computation.

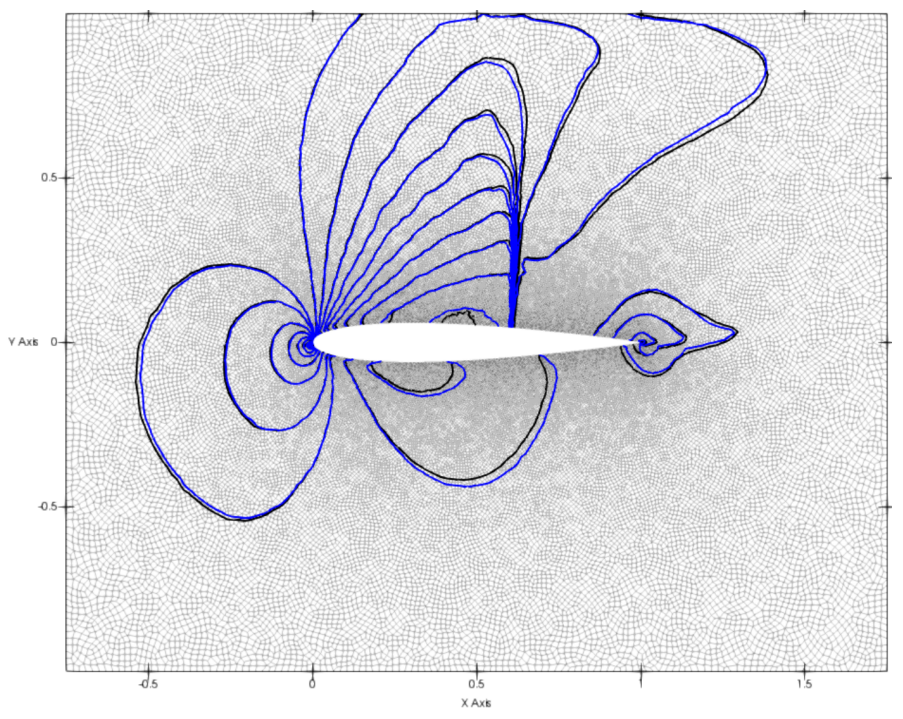

Figure 15: Isolines of the Mach number obtained on the test case described in subsubsection 4.2.4 for the Roe scheme (black) and the Roe scheme corrected with the new fix (blue). 20 isolines were drawn, between 0.1 and 1.4 .

\section{Conclusion}

The aim of this article was to perform low Mach number acoustic computations with density based solvers on the barotropic Euler equations. For that, we need a numerical scheme that is not only able to accurately solve stationary problems, for avoiding instabilities in the base flow, but also able to perform accurate acoustic computations. Different low Mach number fixes were tested. All of them proved to be inaccurate for acoustic computations:

- The Roe-Turkel scheme [23] damps acoustics very quickly.

- The fixes of Dellacherie et al. [12] and Rieper [47] feature a degraded CFL number stability criterion and do not succeed in reaching the expected optimal order for a second order discontinuous Galerkin method.

By using a two-time scale asymptotic expansion, the numerical results obtained with these schemes were explained. Last, a new fix was developed, which can

- converge for the stationary problems,

- and give the optimal order for low Mach number acoustic problems. 
A direct extension of this work is the computation of acoustic problems with the full Euler system. We are also actively working on the extension of this approach to very high order discontinuous Galerkin method.

Acknowledgment: Experiments presented in this paper were carried out using the PLAFRIM experimental tested, being developed under the Inria PlaFRIM development action with support from Bordeaux INP, LABRI and IMB and other entities: Conseil Régional d'Aquitaine, Université de Bordeaux and CNRS (and ANR in accordance to the programme d'investissements d'Avenir (see http://www.plafrim.fr/)).

\section{References}

[1] M. Ainsworth, P. Monk, and W. Muniz. Dispersive and dissipative properties of discontinuous Galerkin finite element methods for the second-order wave equation. Journal of Scientific Computing, 27(1):5-40, Jun 2006.

[2] D. Amenga-Mbengoue, D. Genet, C. Lachat, E. Martin, M. Mogé, V. Perrier, F. Renac, F. Rué, and M. Ricchiuto. Comparison of high order algorithms in Aerosol and Aghora for compressible flows. In ESAIM: Proceedings, volume 43, pages 1-16. EDP Sciences, 2013.

[3] W. Barsukow, P. V. Edelmann, C. Klingenberg, F. Miczek, and F. K. Röpke. A numerical scheme for the compressible low-Mach number regime of ideal fluid dynamics. Journal of Scientific Computing, 72(2):623-646, 2017.

[4] F. Bassi, C. De Bartolo, R. Hartmann, and A. Nigro. A discontinuous Galerkin method for inviscid low Mach number flows. J. Comput. Phys., 228(11):3996-4011, 2009.

[5] P. Birken and A. Meister. Stability of preconditioned finite volume schemes at low Mach numbers. BIT Numerical Mathematics, 45(3):463-480, 2005.

[6] F. Bouchut, C. Chalons, and S. Guisset. An entropy satisfying two-speed relaxation system for the barotropic Euler equations. Application to the numerical approximation of low Mach number flows. working paper or preprint, Dec. 2017.

[7] E. Burman, A. Ern, and M. A. Fernández. Explicit Runge-Kutta schemes and finite elements with symmetric stabilization for first-order linear PDE systems. SIAM Journal on Numerical Analysis, 48(6):2019-2042, 2010.

[8] C. Chalons, M. Girardin, and S. Kokh. An all-regime Lagrange-Projection like scheme for the gas dynamics equations on unstructured meshes. Communications in Computational Physics, 20(1):188-233, 2016.

[9] B. Cockburn and C.-W. Shu. Runge-Kutta discontinuous Galerkin methods for convectiondominated problems. Journal of scientific computing, 16(3):173-261, 2001.

[10] P. Degond and M. Tang. All speed scheme for the low Mach number limit of the isentropic Euler equations. Communications in Computational Physics, 10(1):1-31, 2011.

[11] S. Dellacherie. Analysis of Godunov type schemes applied to the compressible Euler system at low Mach number. Journal of Computational Physics, 4(229):978-1016, 2010.

[12] S. Dellacherie, J. Jung, P. Omnes, and P.-A. Raviart. Construction of modified Godunov type schemes accurate at any Mach number for the compressible Euler system. Mathematical Models and Methods in Applied Sciences, Nov. 2016.

[13] S. Dellacherie, P. Omnes, and F. Rieper. The influence of cell geometry on the Godunov scheme applied to the linear wave equation. Journal of Computational Physics, 229(14):5315-5338, 2010.

[14] S. Delmas. Simulation d'écoulements pariétaux génériques à bas nombre de Mach pour l'amélioration du refroidissement des chambres de combustion aéronautiques. $\mathrm{PhD}$ thesis, Université de Pau et des pays de l'Adour, 2015.

[15] G. Dimarco, R. Loubère, and M.-H. Vignal. Study of a new asymptotic preserving scheme for the Euler system in the low Mach number limit. SIAM J. Sci. Comput., 39(5):A2099-A2128, 2017. 
[16] L. Fezoui and B. Stoufflet. A class of implicit upwind schemes for Euler simulations with unstructured meshes. Journal of Computational Physics, 84(1):174-206, 1989.

[17] J. L. Florenciano Merino. Étude de la réponse d'un écoulement avec transfert pariétal de masse à un forçage acoustique: application au refroidissement des chambres de combustion aéronautiques. PhD thesis, Pau, 2013.

[18] C. Geuzaine and J.-F. Remacle. Gmsh: A 3-d finite element mesh generator with built-in pre-and post-processing facilities. International journal for numerical methods in engineering, 79(11):1309-1331, 2009.

[19] S. Gottlieb, D. I. Ketcheson, and C.-W. Shu. Strong stability preserving Runge-Kutta and multistep time discretizations. World Scientific, 2011.

[20] P. M. Gresho and S. T. Chan. On the theory of semi-implicit projection methods for viscous incompressible flow and its implementation via a finite element method that also introduces a nearly consistent mass matrix. part 2: Implementation. International Journal for Numerical Methods in Fluids, 11(5):621-659, 1990.

[21] H. Guillard. On the behavior of upwind schemes in the low Mach number limit. IV: P0 approximation on triangular and tetrahedral cells. Computers \& Fluids, 38(10):1969-1972, 2009.

[22] H. Guillard and B. Nkonga. On the behaviour of upwind schemes in the low Mach number limit: A review. Handbook of Numerical Analysis, 18:203-231, 2017.

[23] H. Guillard and C. Viozat. On the behaviour of upwind schemes in the low Mach number limit. Computers \& Fluids, 28(1):63-86, 1999.

[24] D. Iampietro, F. Daude, P. Galon, and J.-M. Hérard. A Mach-sensitive implicit-explicit scheme adapted to compressible multi-scale flows. Journal of Computational and Applied Mathematics, 340:122-150, Oct. 2018.

[25] D. Iampietro, F. Daude, P. Galon, and J.-M. Hérard. A Mach-sensitive splitting approach for Euler-like systems. ESAIM: Mathematical Modelling and Numerical Analysis, 2018.

[26] G. B. Jacobs, D. A. Kopriva, and F. Mashayek. A conservative isothermal wall boundary condition for the compressible Navier-Stokes equations. Journal of Scientific Computing, 30(2):177-192, Feb 2007.

[27] W. S. Janna. 5.8 internal incompressible viscous flow. The Handbook of Fluid Dynamics, page 62, 1998.

[28] S. Klainerman and A. Majda. Singular limits of quasilinear hyperbolic systems with large parameters and the incompressible limit of compressible fluids. Communications on pure and applied Mathematics, 34(4):481-524, 1981.

[29] T. Kloczko, C. Corre, and A. Beccantini. Low-cost implicit schemes for all-speed flows on unstructured meshes. Internat. J. Numer. Methods Fluids, 58(5):493-526, 2008.

[30] S. LeMartelot, B. Nkonga, and R. Saurel. Liquid and liquid-gas flows at all speeds. J. Comput. Phys., 255:53-82, 2013.

[31] X.-S. Li and C.-W. Gu. An all-speed Roe-type scheme and its asymptotic analysis of low Mach number behaviour. Journal of Computational Physics, 227(10):5144-5159, 2008.

[32] X.-S. Li and C.-W. Gu. Mechanism of Roe-type schemes for all-speed flows and its application. Computers \& Fluids, 86:56 - 70, 2013.

[33] X.-S. Li, C.-W. Gu, and J.-Z. Xu. Development of Roe-type scheme for all-speed flows based on preconditioning method. Computers \&f Fluids, 38(4):810-817, 2009.

[34] R. Magnus and H. Yoshihara. Inviscid transonic flow over airfoils. AIAA Journal, 8(12):21572162, 1970.

[35] F. Miczek, F. K. Röpke, and P. V. Edelmann. New numerical solver for flows at various Mach numbers. Astronomy \& Astrophysics, 576:A50, 2015. 
[36] Y. Moguen, S. Delmas, V. Perrier, P. Bruel, and E. Dick. Godunov-type schemes with an inertia term for unsteady full Mach number range flow calculations. J. Comput. Phys., 281:556$590,2015$.

[37] B. Müller. Low Mach number asymptotics of the Navier-Stokes equations and numerical implications. In VKI Lecture Series on Computational fluid dynamics, pages 1-52, 1999.

[38] A. Nigro, C. De Bartolo, R. Hartmann, and F. Bassi. Discontinuous Galerkin solution of preconditioned Euler equations for very low Mach number flows. Internat. J. Numer. Methods Fluids, 63(4):449-467, 2010.

[39] A. Nigro, S. Renda, C. De Bartolo, R. Hartmann, and F. Bassi. A high-order accurate discontinuous Galerkin finite element method for laminar low Mach number flows. Internat. J. Numer. Methods Fluids, 72(1):43-68, 2013.

[40] S. Noelle, G. Bispen, K. R. Arun, M. Lukáčová-Medvid'ová, and C.-D. Munz. A weakly asymptotic preserving low Mach number scheme for the Euler equations of gas dynamics. SIAM J. Sci. Comput., 36(6):B989-B1024, 2014.

[41] K. Oß wald, A. Siegmund, P. Birken, V. Hannemann, and A. Meister. L2 Roe: a low dissipation version of Roe's approximate Riemann solver for low Mach numbers. Internat. J. Numer. Methods Fluids, 81(2):71-86, 2016.

[42] M. Parisot and J.-P. Vila. Centered-potential regularization for the advection upstream splitting method. SIAM J. Numer. Anal., 54(5):3083-3104, 2016.

[43] M. Pelanti and K.-M. Shyue. A mixture-energy-consistent six-equation two-phase numerical model for fluids with interfaces, cavitation and evaporation waves. J. Comput. Phys., 259:331$357,2014$.

[44] S. Peluchon, G. Gallice, and L. Mieussens. A robust implicit-explicit acoustic-transport splitting scheme for two-phase flows. Journal of Computational Physics, 339:328-355, 2017.

[45] L. Pesch and J. J. W. van der Vegt. A discontinuous Galerkin finite element discretization of the Euler equations for compressible and incompressible fluids. J. Comput. Phys., 227(11):5426-5446, 2008.

[46] F. Rieper. On the dissipation mechanism of upwind-schemes in the low mach number regime: A comparison between Roe and HLL. Journal of Computational Physics, 229(2):221-232, 2010.

[47] F. Rieper. A low-Mach number fix for Roe's approximate Riemann solver. Journal of Computational Physics, 230(13):5263-5287, 2011.

[48] F. Rieper and G. Bader. The influence of cell geometry on the accuracy of upwind schemes in the low Mach number regime. Journal of Computational Physics, 228(8):2918-2933, 2009.

[49] S. Schochet. Fast singular limits of hyperbolic pdes. Journal of differential equations, 114(2):476-512, 1994. 